\title{
QUELQUES OXYUROIDEA DE MYRIAPODES
}

\author{
Par Robert-Ph. DOLLFUS
}

Des Nématodes se rencontrent fréquemment chez les Myriapodes ; ce ne sont pas des parasites rares et exceptionnels. Beaucoup de zoologistes, de protistologistes, et non des moindres, ont eu l'occasion de disséquer des Myriapodes, soit pour l'étude des Myriapodes eux-mêmes, soit pour y chercher des Grégarines ou d'autres Protozoaires, ou des Eccrinides ; ils n'ont pas manqué d'y rencontrer des Nématodes; ils les ont vraisemblablement conservés pour les soumettre à des helminthologistes, comme c'était leur devoir de bons naturalistes, mais bien peu d'entre eux ont dit ce qu'étaient devenus ces matériaux; le plus souvent même, ils n'y ont fait aucune allusion dans leurs publications. La plupart des, ouvrages généraux sur les Myriapodes passent sous silence le parasitisme de ceux-ci par des Nématodes. C'est tout juste si H. W. Brölemann (1935, p. 83, Faune de France, $\mathrm{n}^{\circ} 29$, Diplopodes Chilognathes) (1), savant pourtant éminent, leur a, une fois, consacré trois lignes ; le Capt. J. L. Cloudsley-Thompson (1949, p. 140) (1) s'est contenté d'indiquer à leur sujet quatre références bibliographiques. Il faut donc savoir gré aux quelques zoologistes non helminthologistes ayant pris soin de signaler leurs récoltes de Nématodes au cours de leurs recherches sur des Myriapodes.

Johannes Bode (1878, p. 235) relate avoir une fois trouvé un petit Nématode dans Polyxenus lagurus De Geer, à Halle (Saxe). Otto vom Rath (1891, p. 191) dit avoir observé des Mermis et de jeunes Nématodes dans des Polydesmides et des Julides. Filippo Silvestri, ayant récolté quelques Nématodes chez des Diplopodes de l'île Mentawei, de Sumatra et de Palerme (Sicile), les remit à Corrado Parona (1896), qui en donna la description. W. Wernitzsch (1910, p. 249), 141 .

(1) The Enemies of Myriapods. The Naturalịst, London, oct.-déc. 1949, p. 137-

On trouvera une bibliographie assez importante sur les Nématodes de Myriapodes dans: Paul-A. RÉry, On the enemies of Myriapods. The Naturalist, London, july-sept. 1950 , p. 103-108.

Nous apprenons par P.-A. RÉmy $(1950$, p. 104) que, chez un Symphyle : Scutigerella immaculata Newport 1845 , un Nématode, qui n'a pas été identifié, a été trouvé par Henri Ribaut (en France vraisemblablement); nous espérons que ce Nématode a été conservé.

Ann. de Parasitologie, T. XXVII, $\mathrm{N}^{\circ} 1-2-3 .-1952$. 
à propos de son étude de Craspedonema simile Verhoeff, des environs d'Iéna, dit qu'il y a de jeunes Nématodes dans l'intestin des Craspedonematidés.

Plus récemment, Otto Schubart (1947, p. 11, 18, 19, 22, 27, 29, 33, $34,67,72-73)$ a relaté avoir récolté des Nématodes dans l'intestin de huit espèces de Diplopodes de l'Etat de Mato-Grosso (Brésil) et les avoir envoyés à Lauro Travassos ; on peut donc espérer qu'ils seront tôt ou tard étudiés (1).

Dans la thèse de A. S. Sanchez sur les Nématodes des Arthropodes d'Espagne, on lit $(1946$, p. 11, $26 ; 1947$, p. 292) que cet auteur a trouvé une espèce nouvelle de Nématode chez Julus terrestris L., mais la description n'en a pas, jusqu'à présent, été publiée.

Le peu que l'on sait sur les Nématodes des Myriapodes, on le doit tout d'abord à deux précurseurs : Joseph Leidy (1849-1856) et Jules d'Udekem (1859), ayant chacun publié un travail qui fait encore autorité sur le sujet. Furent ensuite publiées quelques descriptions trop sommaires par O. v. Linstow $(1878,1885)$, C. Parona (1896), Adolf Meyer (1896); puis des travaux montrant une meilleure connaissance du sujet par N. A. Cobb $(1898,1929)$, G. Gilson (1898), K. I. Skrjabin $(1916,1927)$, J. R. Christie et N. A. Cobb (1927), P. Artigas $(1926,1929,1930)$, L. Travassos (1929), L. J. Thomas (1930), B. G. Chitwood (1930), M. B. Chitwood (1935), J. L. de Almeida (1933) (2).

Pour étudier les Nématodes des Myriapodes, il ne suffit évidemment pas de se contenter de prendre connaissance de la description des espèces récoltées chez des Myriapodes. Il y a, en effet, chez ceux-ci, des Nématodes appartenant à des genres et même à des

(1) Ces Diplopodes sont: Ganguia tapirapensis O. Schubart 1947, de Barra do Tapirapé (Mato Grosso) ; Leptodesmus coriaceus O. Schubart 1947, de Aldeia dos Tapirapés (Mato Grosso); Rhinocricus urukumui O. Schubart 1947, de Barra do Tapirapé (Mato Grosso); Stenostreptus unilineatus O. Schubart 1945, de Barra do Tapirapé (Mato Grosso) ; Stenostreptus carvalhoi O. Schubart 1947, de Barra do Tapirapé (Mato Grosso); Stenostreptus tampiitanensis O. Schubart 1947, de Aldeia dos Tapirapés (Mato Grosso) ; Conchostreptus pictus O. Schubart 1945, de Barra do Tapirapé (Mato Grosso) ; Orthoporus araguayensis o. Schubart 1947, de Barra do T'apirapé (Mato Grosso). Certains individus du Leptodesmus et du Rhinocricus contenaient environ 100 nématodes, ou plus de 100.

(2) J'ai rassemblé dans le tableau des pages 147-154 les observations publiées de Nématodes trouvés chez des Myriapodes. Ce tableau ne concerne pas les Nématomorphes (Gordiens), dont plusieurs espèces ont été signalées ou décrites chez les Myriapodes (références bibliographiques, jusqu'en 1936, in P.-A. RÉmy, 1950 , p. 108 ; voir aussi J.-C.-M. Carvalho, 1946, p. 2). Je n'ai pas non plus tenu compte de l'observation d'œufs de Nématodes accidentellement ingérés par des Myriapodes avec leur nourriture. O. von Lisstow (1886, p. 527) a constaté que Julus guttulatus Bosc et Polydesmus complanaĩus (L.) avalaient volontiers des œufs d'Ascaris lumbricoides L. déposés dans la terre d'un terrarium. Dans le contenu intestinal d'un Julus de Banyuls (Pyrénées-Orientales), Jean THÉODoRIDÈs m'a montré un jeune Nématode détriticole ou parasite de plante, pourvu d'un stylet buccal ; il avait été évidemment avalé avee des débris de végétaux. 
espèces qui se rencontrent aussi chez des Insectes. Ce fait semble maintenant bien établi. Par exemple, Aorurus agile (J. Leidy 1849) (fam. Thelastomatidæ), de Julus (Spirobolus) marginatus Say, est aussi hébergé par la larve d'un Coléoptère Scarabéide, Osmoderma sp. [O. scabra Beauvois ?] ; des spécimens provenant de ce dernier hôte avaient été décrits sous un nom différent: Aorurus subclavatus J. R. Christie 1931, mais B. G. Chitwood et M. B. Chitwood (1934, p. 384,387 ) reconnurent qu'il s'agissait de la même espèce ; ce que confirma J. R. Christie (1938, p. 67), l'examen des originaux d'agile (collection J. Leidy) lui ayant montré que l'emplacement indiqué par J. Leidy pour la vulve était inexact.

Si donc la famille Thelastomatidæ (L. Travassos 1929) compte des représentants chez des Myriapodes, elle en compte aussi chez des Insectes, quelquefois chez des Coléoptères et chez d'autres groupes, mais beaucoup plus communément chez des Blattes. Deux autres familles sont représentées chez les Myriapodes : les Atractidæ et les Rhigonematidæ; il semble que les genres de Rhigonematidæx se rencontrent seulement chez des Myriapodes et que ceux des genres d'Atractidæ observés chez les Myriapodes appartiennent à une sous-famille ne comptant pas de représentants en dehors des Myriapodes.

\title{
Familles, sous-familles et genres de la superfamille Oxyuroidea A. Railliet 1905, représentés chez les myriapodes
}

\author{
Fam. THELASTOMATIDA (L. Travassos 1929).
}

Sous-fam. THELASTOMATINE (L. Travassos 1920)

(incl. Aorurinæ A. C. Walton 1928)

Gen. Thelastoma (J. Leidy 1849) J. Leidy $1853 \quad 1$ ou 0 spicule.

Gen. Aorurus J. Leidy 1849 ............

Gen. Severianoia (J. Schwenck 1926 subgen.) 1 spicule.

L. Travassos $1929 \ldots \ldots \ldots \ldots \ldots \ldots \ldots \ldots$
en. Fontonema B. G. Chitwood $1930 \ldots \ldots$ o o inconnu.

Fam. RHIGONEMATIDE (P. Artigas 1930)

Sous-fam. RHIGONEMATINAE (P. Artigas 1930)

Gen. Rhigonema N. A. Cobb 1898 ....... 2 spicules égaux.

(incl. s. g. Dudekemia (P. Artigas 1930 gen.)

Ann. de Parasitologie, T. XXVII, N ${ }^{\circ} 1-2-3 .-1952$.

10. 
Sous-fam. ICHTHYOCEPHALINAE P. Artigas 1930

Gen. Ichthyocephalus P. Artigas 1926 .... 2 spicules égaux.

Fam. ATRACTIDA (L. Travassos 1920)

Sous-fam. RANSOMNEMATINE (L. Travassos 1929)

Gen. Ransomnema P. Artigas $1926 \ldots \ldots \ldots 2$ spicules inégaux.

Gen. Rondonema P. Artigas $1926 \ldots \ldots \ldots .2$ spicules égaux +

1 gubernaculum.

Gen. Heth N. A. Cobb $1898 \ldots \ldots \ldots \ldots \ldots \ldots 2$ spicules inégaux (ou 1 spicule + 1 gubernaculum)

Gen. Angra L. Travassos $1929 \ldots \ldots \ldots \ldots \ldots 2$ spicules égaux fusionnés +1 gubernaculum.

Gen. Carnoya G. Gilson $1898 \ldots \ldots \ldots \ldots \ldots 2$ spicules égaux + 1 gubernaculum.

Gen. Clementeia P. Artigas $1930 \ldots \ldots \ldots \ldots 2$ spicules égaux + 1 gubernaculum.

Sous-fam. CRUZNEMATINAE (P. Artigas 1929)

Gen. Cruznema P. Artigas 1927 ......... o inconnu.

Une ventouse. préanale existe seulement chez les ố des trois genres : Ransomnema, Clementeia, Heth-Streptogaster.

Fam. THELASTO.IATIDA (L. Travassos 1929)

(incl. Lepidonematidæe (L. Travassos 1920)

Selon B. G. Chitwood (1932, p. 16), cette famille comprend trois sous-familles : Thelastomatinæ (L. Travassos 1929) (incl. Aorurinæ A. C. Walton 1927), Hystrignathinæ L. Travassos 1920 (1) (incl. Lepidonematinæ (L. Travassos 1920), Protrelloidinæ B. G. Chitwood 1932.

Une seule de ces sous-familles est représentée, jusqu'à présent, chez les Myriapodes.

(1) Dans la classification de J.-R. Christie (1934, p. 44-45), il n'y a pas de sous-famille Hystrignathina et Hystrignathus est placé dans la sous-famille Thelastomatina comme Artigasia J.-R. Christie 1934, et Lepidonema N.-A. Cobb 1898. 


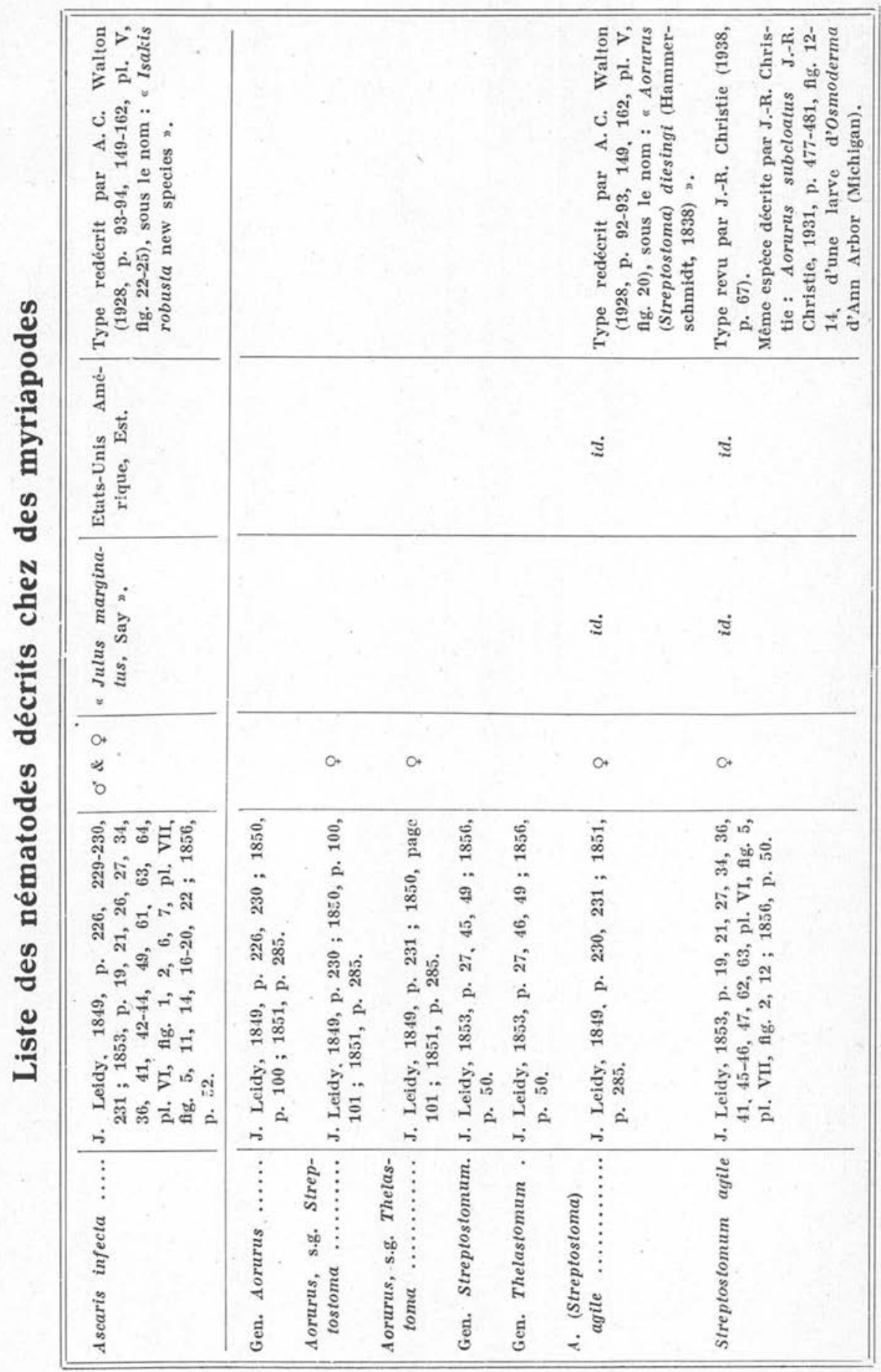




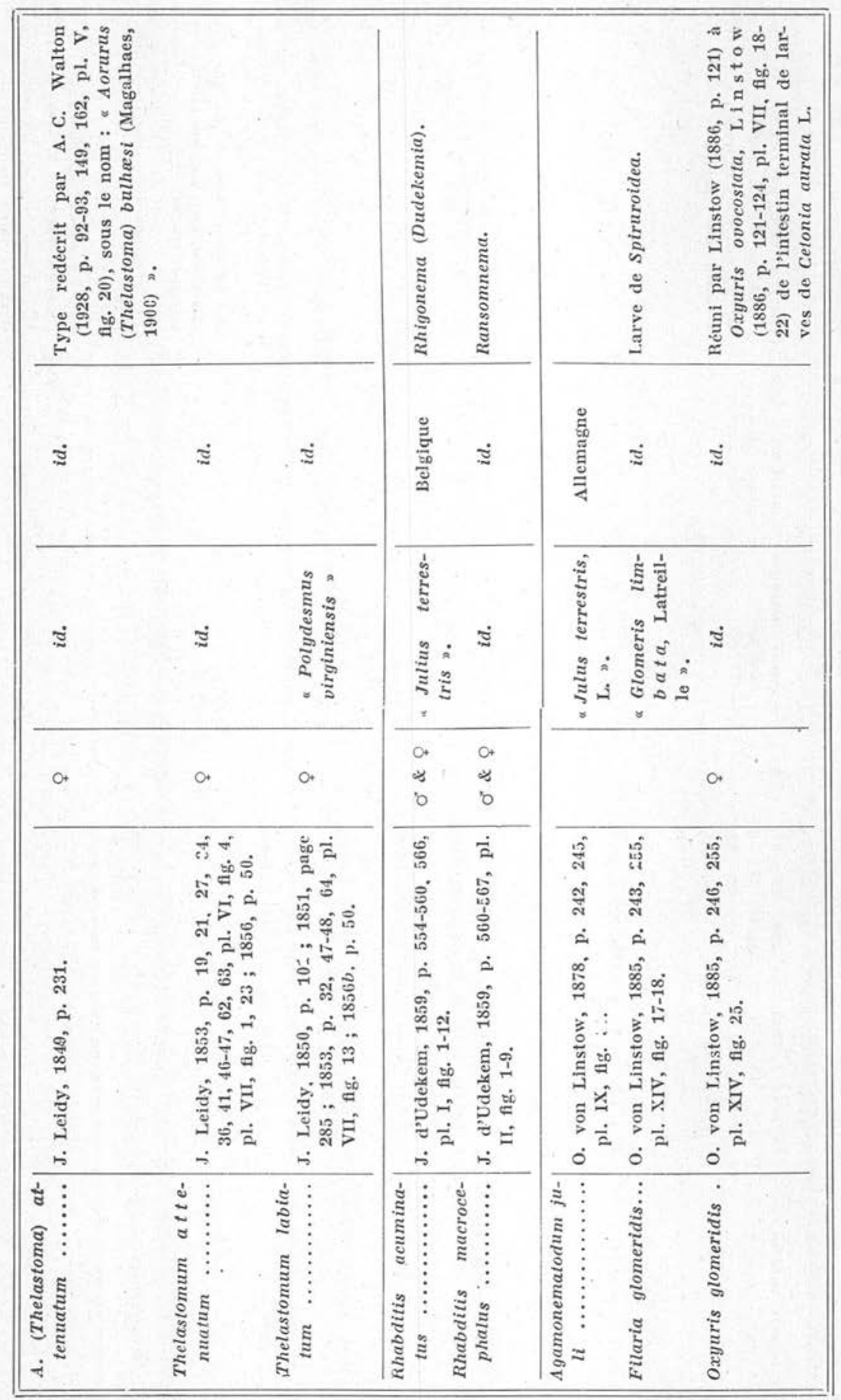




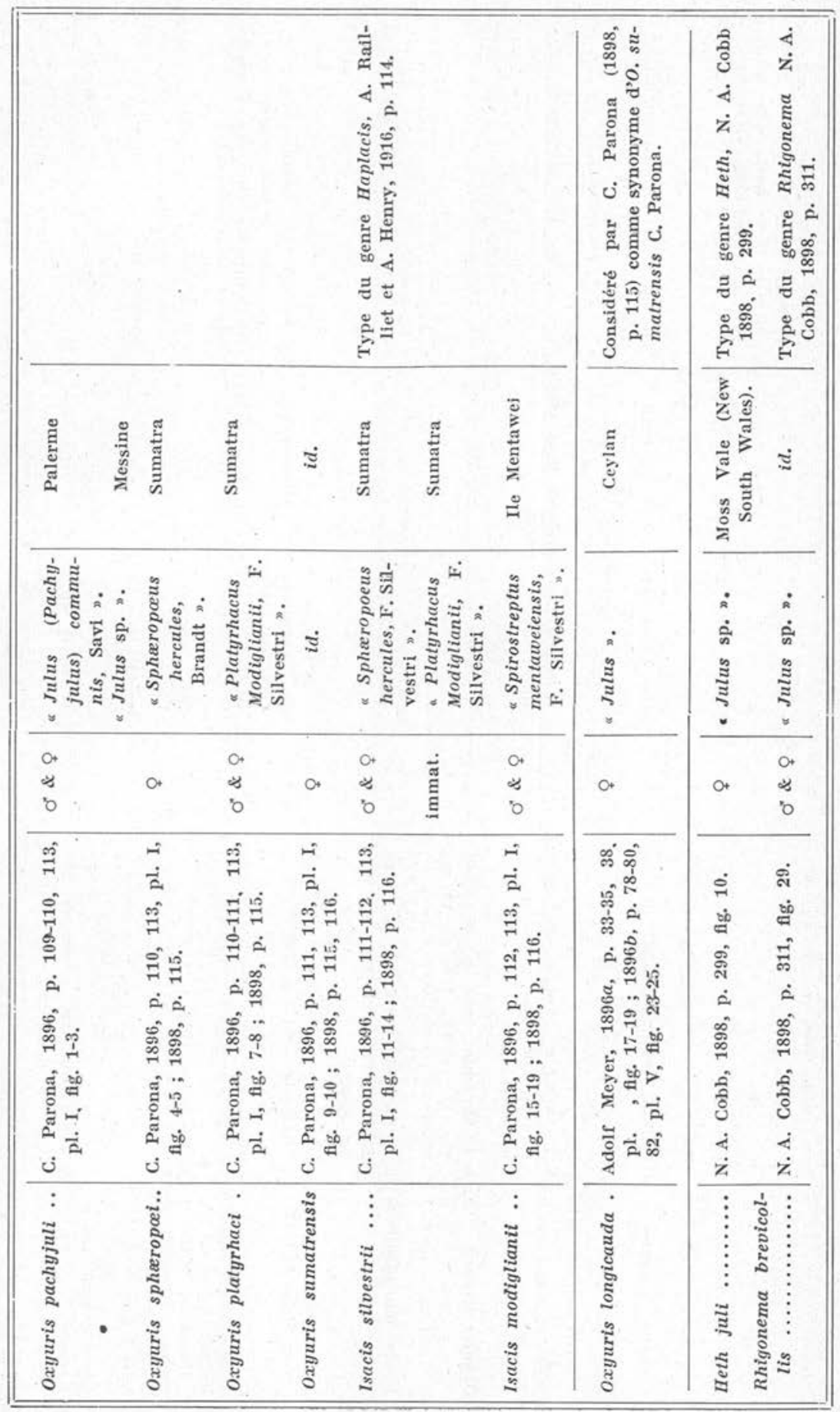




\begin{tabular}{|c|c|c|c|c|c|c|c|}
\hline 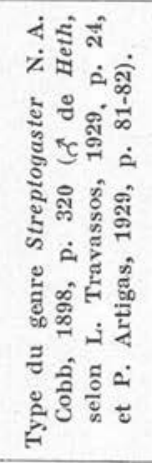 & 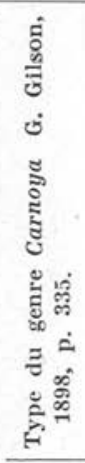 & & & & 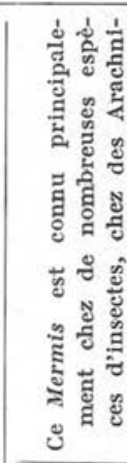 & 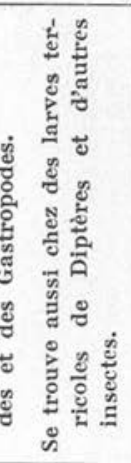 & \\
\hline$\approx$ & $\begin{array}{l}\widehat{\bar{Z}} \\
\text { 플 } \\
\text { II } \\
\text { \# }\end{array}$ & 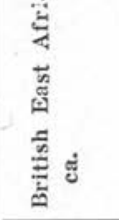 & $\dot{z}$ & $\dot{z}$ & 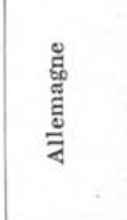 & $\dot{\Xi}$ & 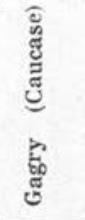 \\
\hline \% & 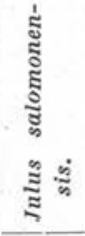 & 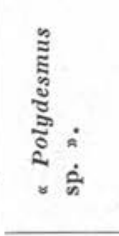 & $\dot{z}$ & 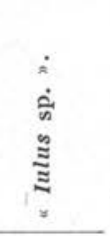 & 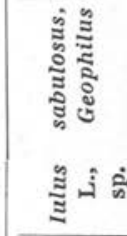 & 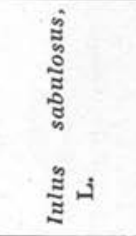 & 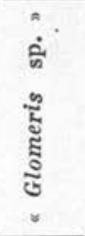 \\
\hline 0 & $\begin{array}{l}\alpha \\
* \\
0\end{array}$ & o. & a. & $\begin{array}{l}\alpha \\
* \\
0\end{array}$ & $\stackrel{D}{E}$ & & $\begin{array}{l}0 . \\
* \\
y\end{array}$ \\
\hline 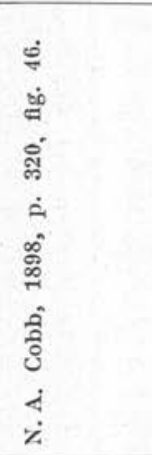 & 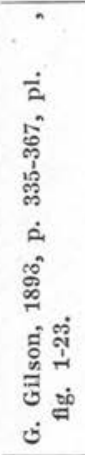 & 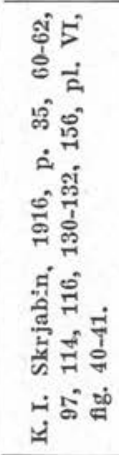 & 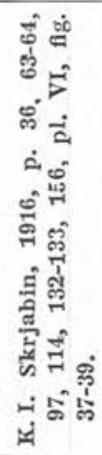 & 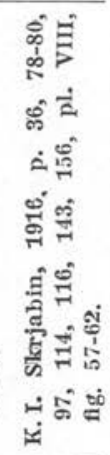 & 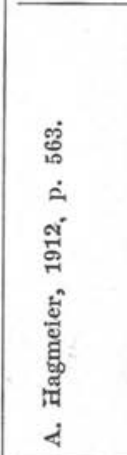 & 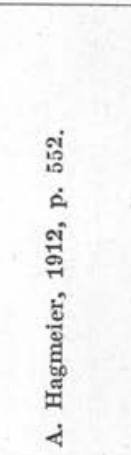 & 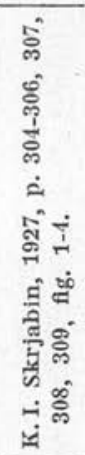 \\
\hline 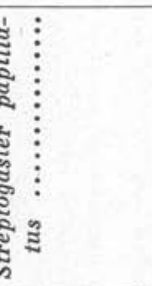 & 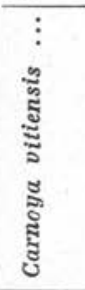 & 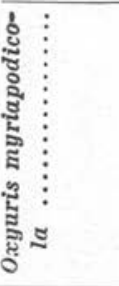 & 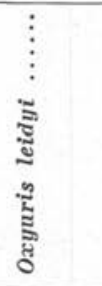 & 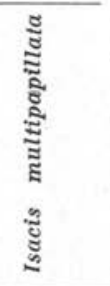 & 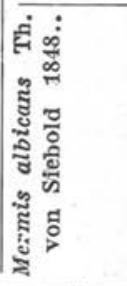 & 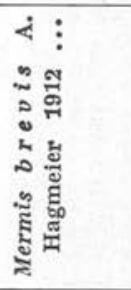 & 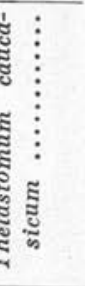 \\
\hline
\end{tabular}




\begin{tabular}{|c|c|c|c|c|c|c|c|}
\hline 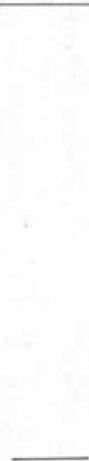 & 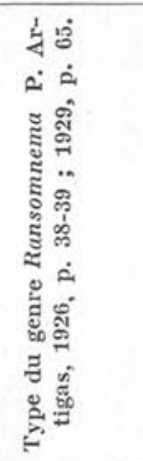 & & 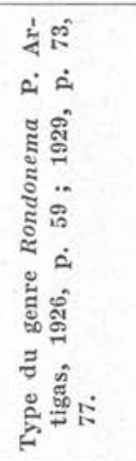 & 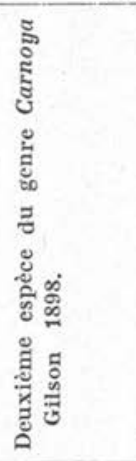 & 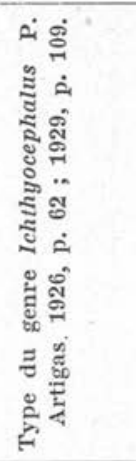 & 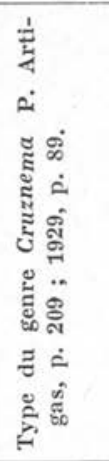 & \\
\hline 竎 & 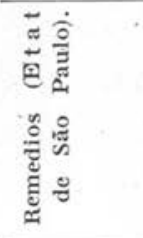 & 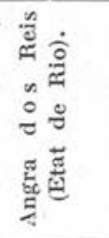 & 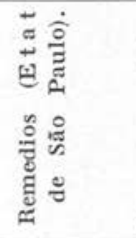 & 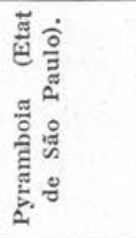 & 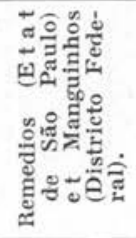 & 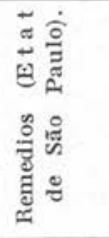 & 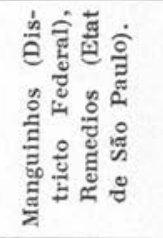 \\
\hline 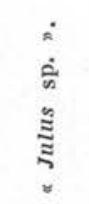 & 节 & $\dot{\Xi}$ & 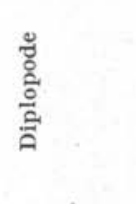 & $\dot{\Xi}$ & $\dot{z}$ & $\dot{\Xi}$ & 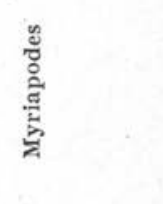 \\
\hline a. & $\begin{array}{l}0 \\
\ddot{z} \\
0\end{array}$ & $\begin{array}{l}0 \\
* \\
0\end{array}$ & $\begin{array}{l}a \\
* \\
0\end{array}$ & $\begin{array}{l}c^{+} \\
\ddot{\sigma} \\
0\end{array}$ & $0+$ & o. & $\begin{array}{l}0 \\
* \\
0\end{array}$ \\
\hline 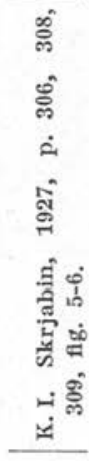 & 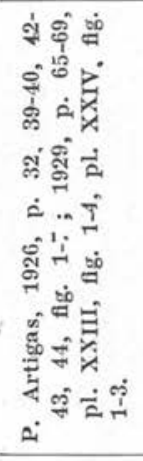 & 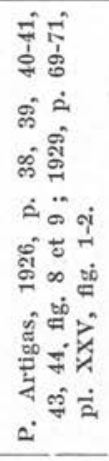 & 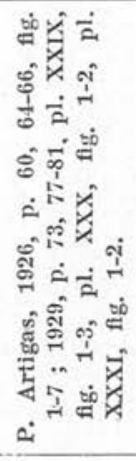 & 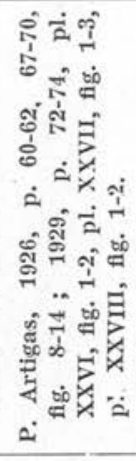 & 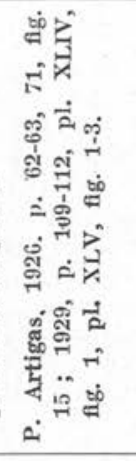 & 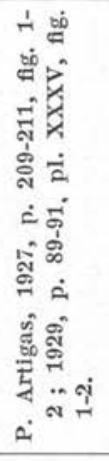 & 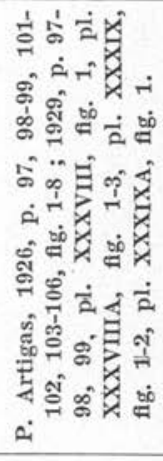 \\
\hline 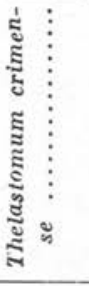 & 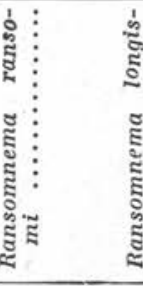 & 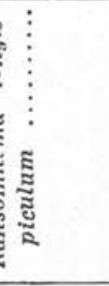 & 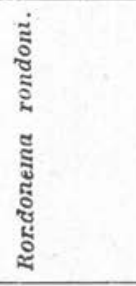 & 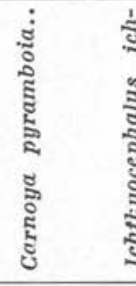 & 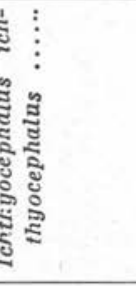 & 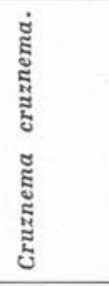 & 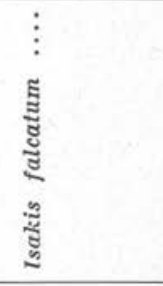 \\
\hline
\end{tabular}




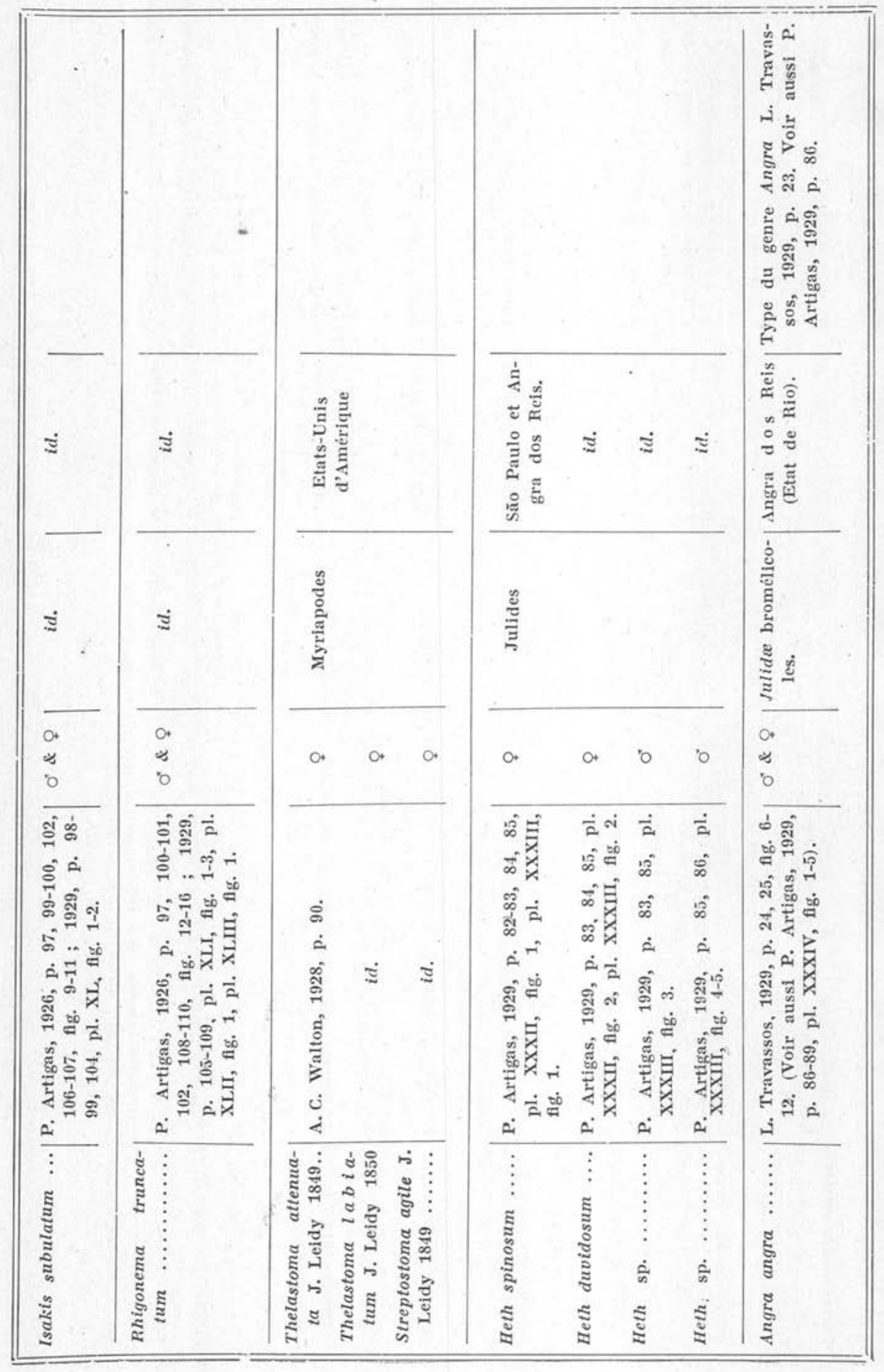




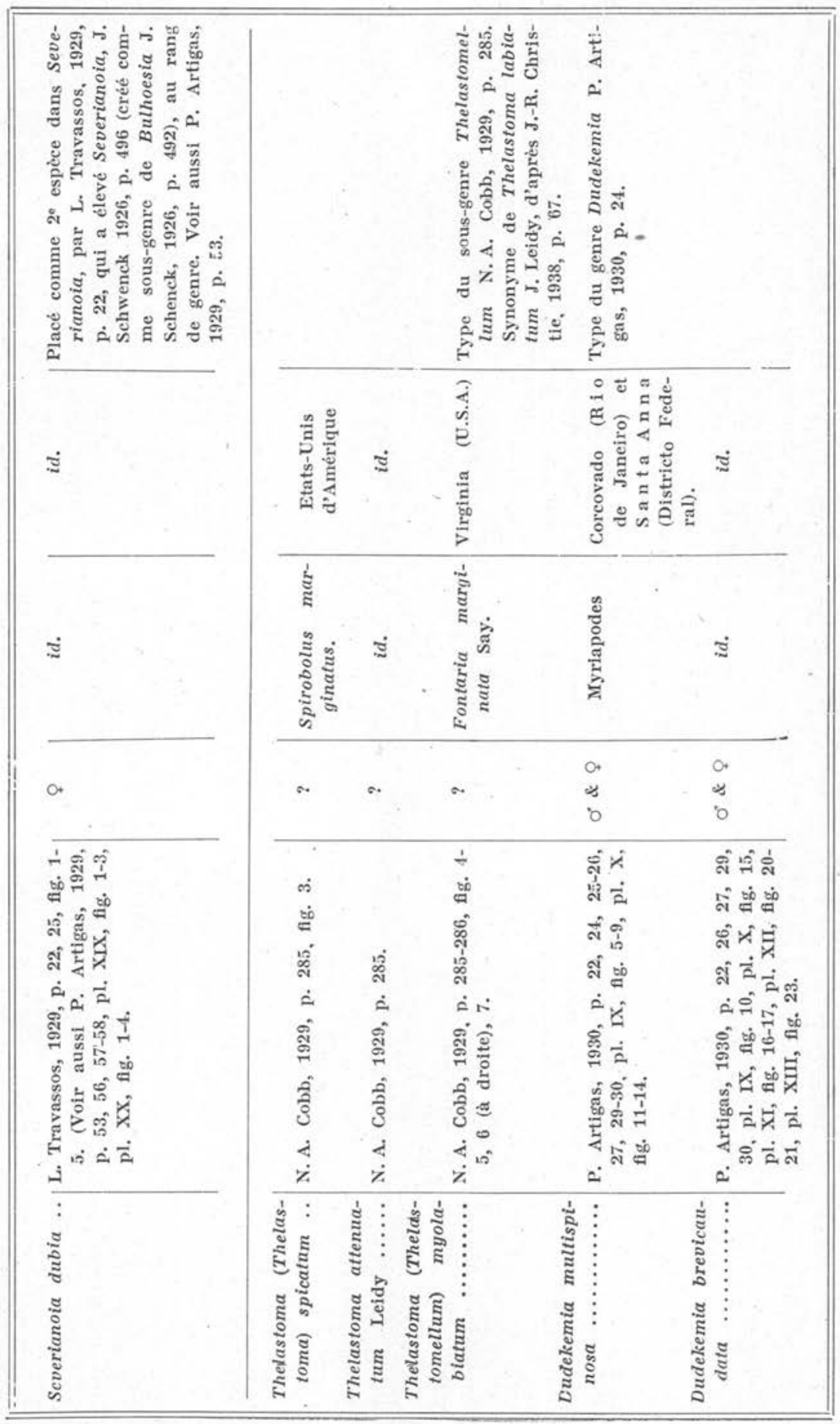




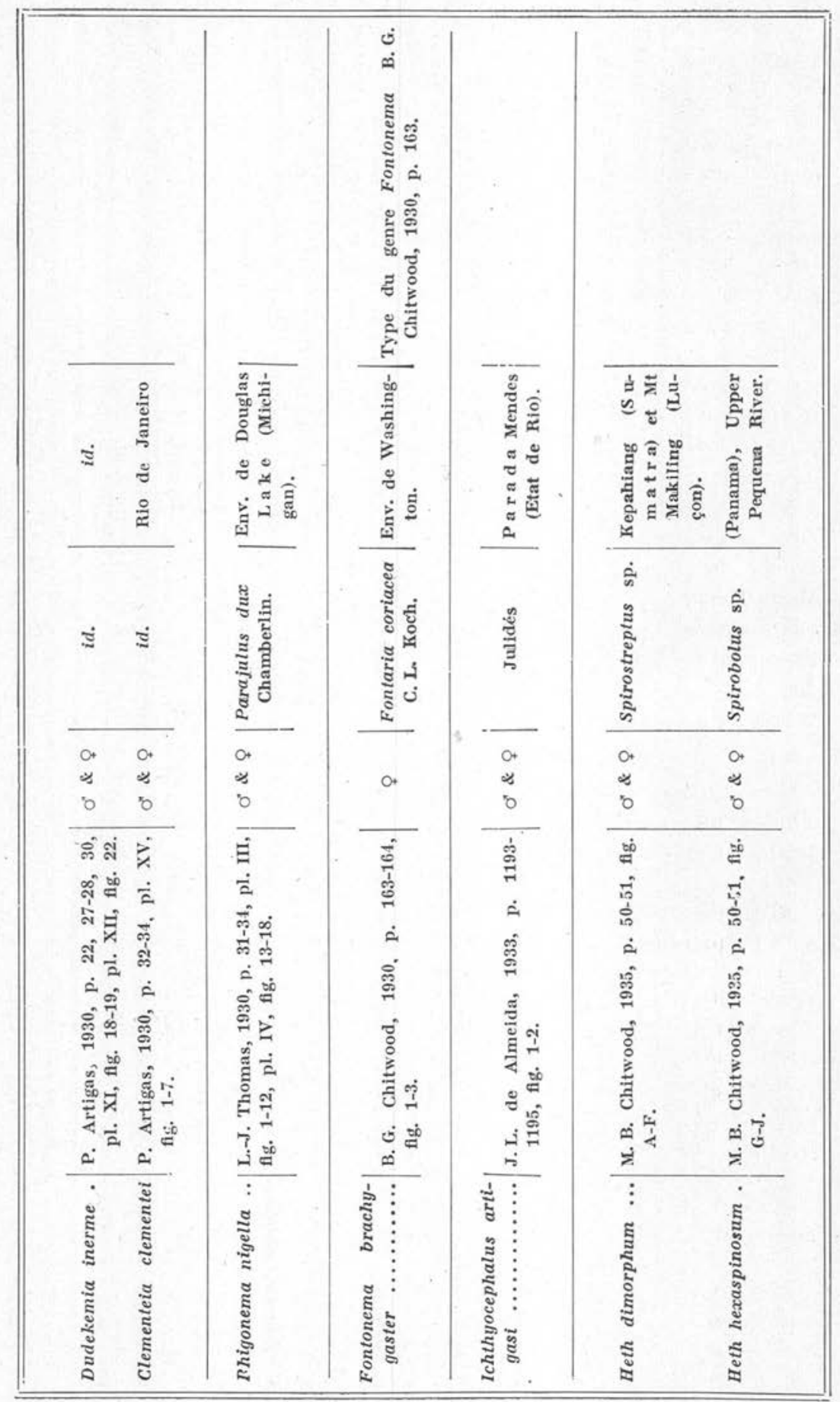




\section{Sous-famille THELASTOMATINAE (L. Travassos 1929)}

La plupart des genres de cette sous-famille sont connus seulement chez des Blattides. Une clef a été donnée par B. G. Chitwood et M. B. Chitwood (1934, p. 391-392) ; elle comprend les 15 genres suivants : Thelastoma J. Leidy 1849 (incl. Bulhoesia J. Schwenck 1926), Aorurus J. Leidy 1849, Pseudonymus K. M. Diesing 1857, Blattophila N. A. Cobb 1920, Cephalobellus N. A. Cobb 1920, Binema L. Travassos 1925, Blatticola J. Schwenck 1926, Leidynema J. Schwenck in L. Travassos 1929, Severianoia J. Schwenck 1926, L. Travassos 1929, Fontonema B. G. Chitwood 1930, Euryconema B. G. Chitwood 1932, Suifunema B. G. Chitwood 1932, Galebia B. G. Chitwood 1932, Hammerschmidtiella B. G. Chitwood 1932, Leidynemella B. G. Chitwood et M. B. Chitwood 1934. Depuis lors, ont été ajoutés : Blattelicola M. A. Basir 1940, Periplaneticola M. A. Basir 1940, Galebiella M. A. Basir 1941, Gryllocola M. A. Basir 1942, Gryllophila M. A. Basir 1942, Talpicola M. A. Basir 1942, Mirzaiella M. A. Basir 1942, Zonothrix A. C. Todd 1942, Cameronia M. A. Basir 1948, Chitwoodiella M. A. Basir 1948 ; en tout, 25 genres; pour les 22 premiers, une clef a été donnée par M. A. Basir (1942, p. 104105).

Pour plusieurs genres, le ô est inconnu ; lorsqu'il est connu, il a un seul spicule ou aucun, jamais de gubernaculum.

Les Thelastomatinæ de Myriapodes ont été placés dans les genres Thelastoma, Aorurus, Severianoia et Fontonema. Ces genres peuvent être distingués d'après les caractères ci-après :

AORURUS. - q. Bouche entourée par un cercle externe de 8 papilles ou labiopapilles. Première partie de l'œsophage piriforme (courte et se dilatant beaucoup postérieurement), suivie, avec démarcation, par un isthme étroit, long ou court, se continuant, avec démarcation, par le goulot du bulbe. Vulve, soit très voisine de l'anus, soit près du milieu du corps. Queue filiforme ou presque filiforme.

đ. Bouche comme chez la ㅇ. GEsophage sans dilatation, subcylindrique, se continuant, avec démarcation, par le goulot du bulbe ; 2 paires de papilles, juste en avant de l'anus (dont 1 paire plus ou moins fusionnée), 1 paire de papilles (en partie fusionnées), juste en arrière de l'anus, 1 paire de papilles sur la partie rétrécie de la queue. 1 spicule présent. Queue filiforme.

THELASTOMA. - q. Bouche entourée par un cercle externe de 8 papilles. Première partie de l'œsophage (corpus) cylindriforme, sans dilatation, se continuant, avec démarcation, par le goulot du bulbe. Vulve 
un peu en arrière de la mi-longueur du corps (non compris la queué). Queue filiforme, très longue et grêle.

ð. Bouche non entourée d'élévations papilliformes. Esophage comme chez la f. Ailes latérales présentes ou absentes. 3 paires de papilles sur la protubérance anale, 1 paire de papilles sur la partie rétrécie de la queue. 1 spicule présent ou absent. Queue filiforme ou presque filiforme.

SEVERIANOIA. - - . Bouche sans lèvres saillantes. Essophage sans dilatation, se continuant, avec démarcation, par le goulot du bulbe. Vulve en arrière de la mi-longueur du corps (non compris la queue). Queue courte, presque cônique.

§. Bouche comme chez la ․ Esophage comme chez la ‥ 1 spicule. 1 paire de papilles adanales et 2 paires postanales. Queue comme chez la $\uparrow$, sans papilles.

FONTONEMA. - ․ Bouche entourée par 3 lèvres égales. Esophage très long, sans dilatation, se continuant, avec démarcation, par le goulot du bulbe. Vulve en arrière de la mi-longueur du corps (non compris la queue). Queue longue et filiforme.

o. Inconnu.

THELASTOMA GALLIARDI n. sp.

(Fig. 1-2 et pl. I, fig. 1)

․ Corps subcylindrique, s'atténuant plus lentement antérieurement que postérieurement, se terminant sans brusque rétrécissement par une queue longuement conique et pointue, non filiforme, longue comme approximativement $1 / 8^{\circ}$ de la longueur totale de l'individu. Anneaux cuticulaires bien distincts, le premier est beaucoup moins large que tous les autres, il est plus haut que ceux situés immédiatement après, et forme une tête extérieurement délimitée. Les lèvres buccales ou les labio-papilles circumorales ne paraissent pas saillantes. La cavité buccopharyngienne est un peu plus profonde que large. Le corpus œesophagien est cylindrique, long et plutôt grêle, il se continue, avec démarcation, par un isthme court, à peu près de même diamètre, qui est le goulot du bulbe ; celui-ci est large comme à peu près les deux tiers de la largeur du corps à son niveau. L'extrémité antérieure de l'intestin est un peu plus large que le bulbe. L'anneau nerveux est à la limite entre les deux derniers quarts du corpus œsophagien. Le pore excréteur est situé en arrière du bulbe, à une distance un peu moindre que le diamètre transversal du bulbe. Appareil génital amphidelphe, utérus pouvant contenir environ 55-60 œufs ellipsoïdaux, à coque mince. Vulve non saillante vers la fin du second tiers de la longueur du corps, non compris la queue, 


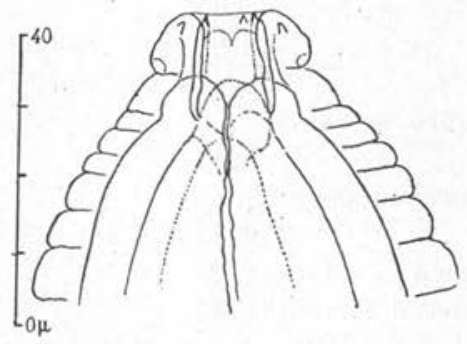

Fig. 1. - Thelastoma Galliardi n. sp. ; intestin de Glomeris. Extrémité antérieure d'une ㅇ. Ipse legi; $\mathrm{Ri}_{-}$ chelieu (Indre-et-Loire), 11-9-1951.

FIG. 2. - Même espèce et même provenance que pour la fig. 1. Région postérieure d'un ơ long de $1 \mathrm{~mm} .4$, large de $0 \mathrm{~mm} .06$.

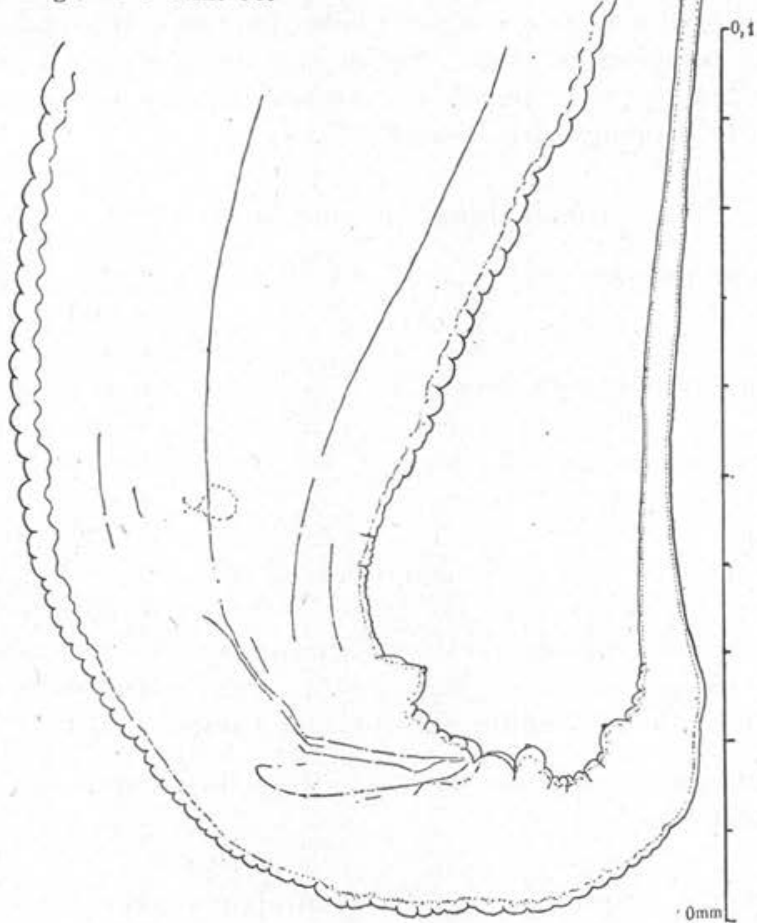

Dimensions, en mm., d'une $q$ à maturité :

Longueur totale ................ 2.142

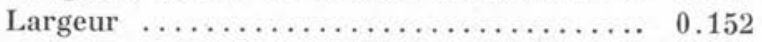

Queue .................... 0.292

Corpus œesophagien, longueur ........ 0.315

largeur $\ldots \ldots \ldots \ldots \ldots, 0.035-0.025$ 
Isthme œsophagien, longueur $\ldots \ldots \ldots \ldots \ldots \quad 0.025$

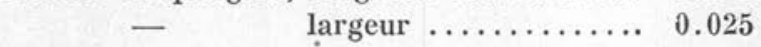

Bulbe..$\ldots \ldots \ldots \ldots \ldots \ldots \ldots \ldots \ldots \ldots$

Distance de l'extrémité antérieure à l'anneau nerveux ....................

Distance de l'extrémité antérieure au pore excréteur .................... 0.525

Distance de l'extrémité antérieure à la vulve 1.24

Distance de l'extrémité antérieure à l'anus 1.85

Eufs $($ en $\mu$ ), 78-86 $\times 52 \mu$; quelquefois $89 \times 46-49 \mu$.

§. Corps subcylindrique s'atténuant postérieurement, sans brusque rétrécissement, en une longue queue devenant presque filiforme, plus longue que le tiers de la longueur totale de l'individu. Annulation cuticulaire bien distincte. Il n'y a pas d'ailes latérales. Esophage cylindrique. Isthme œsophagien court. Aucun spicule. Une paire de grosses papilles préanales, deux paires de grosses papilles postanales, aucune papille sur le prolongement caudal.

Dimensions, en mm., d'un $\delta$ :

\begin{tabular}{|c|c|}
\hline Longueur totale $\ldots \ldots \ldots$. & 1.34 \\
\hline Largeur $\ldots \ldots \ldots \ldots \ldots \ldots \ldots \ldots \ldots \ldots \ldots \ldots \ldots$ & 0.060 \\
\hline 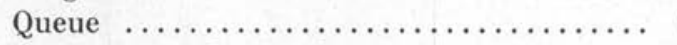 & 0.13 \\
\hline Corpus œsophagien, longueur . ......... & 0.17 \\
\hline largeur $\ldots \ldots \ldots \ldots$ & $0.010-0.014$ \\
\hline Isthme œsophagien, longueur .......... & 0.014 \\
\hline$-\quad$ largeur $\ldots \ldots \ldots \ldots$ & 0.014 \\
\hline Bulbe $\ldots \ldots \ldots \ldots \ldots \ldots \ldots \ldots \ldots \ldots$ & $0.037 \times 0.034$ \\
\hline $\begin{array}{l}\text { Distance de l'extrémité antérieure à l'an- } \\
\text { neau nerveux } \ldots \ldots \ldots \ldots \ldots \ldots \ldots \ldots \ldots \ldots\end{array}$ & $0.092(?)$ \\
\hline $\begin{array}{l}\text { Distance de l'extrémité antérieure au pore } \\
\text { excréteur } \ldots \ldots \ldots \ldots \ldots \ldots \ldots \ldots \ldots \ldots \ldots\end{array}$ & $0.290(?)$ \\
\hline Distance de l'extrémité antérieure à l'anus & 1.210 \\
\hline
\end{tabular}
ingi, 11-9-1951 (1).

Discussion. - Cette espèce est à comparer avec celles du genre dcnt le ठ̊ n'a ni spicule, ni papilles sur le prolongement caudal et dont le pore excréteur est postbulbaire; mais aucune espèce ne m'est connue dont le ỡ réunisse ces caractères. Si l'on cherche parmi

(1) Dans la cavité génerale des mêmes Glomeris, il y avait des amas de capsules de consistance gélatineuse contenant chacune de 1 à 3 larves lovées chacune dans un kyste de $152-162 \mu$ environ de diamètre ; en outre, quelques très petites larves étaient libres; peut-être s'agissait-il de larves de Spiruroidea. L'étude de ces larves n'a pas encore été entreprise. 
les espèces dont le $\delta$ est inconnu et dont la $q$ a le pore excréteur postbulbaire (par exemple icemi et magalhæsi), il n'y en a pas dont les dimensions soient compatibles; j'estime donc que le Thelastoma de Glomeris récolté à Richelieu est une espèce nouvelle, et je la dédie à mon ami, le $\mathrm{D}^{\mathrm{r}}$ Henri Galliard, Directeur de l'Institut de Parasitologie de la Faculté de Médecine de l'Université de Paris et de la Station expérimentale de Richelieu (Indre-et-Loire).

\section{THELASTOMA GLOMERICOLA n. sp.}

(Fig. 3-4 et pl. I, fig. 2)

․ Corps subcylindrique s'atténuant antérieurement et postérieurement, se terminant sans brusque rétrécissement par une queue pointue, sans prolongement filiforme, longue comme environ $1 / 6^{\circ}$ de la longueur totale. L'annulation du corps est bien apparente, mais la hauteur des anneaux est très variable, ce qui est probablement dû, en partie, au fait que la contraction et l'allongement varient selon le niveau. Tête distincte, formée par le premier anneau, plus haut et moins large que les suivants. Les lèvres ne sont pas proéminentes; la disposition des papilles, supposées au nombre de 8 , n'a pas été observée. La cavité buccopharyngienne (stoma) est à peu près aussi longue que large. Le corpus œsophagien est subcylindrique, légèrement atténué antérieurement. L'isthme est moins large que l'extrémité postérieure du corpus, dont il est bien délimité, il est très court, à peu près aussi long que large. Le bulbe est large, comme presque la moitié du corps à son niveau. Le début de l'intestin est fortement dilaté. L'anneau nerveux est à peu près au niveau de l'union des deux derniers tiers de la longueur du corpus œsophagien. Le pore excréteur est en arrière du bulbe, à une distance à peu près égale à la longueur bulbe + isthme. Appareil génital amphidelphe, vulve non saillante, un peu en arrière de la mi-longueur du corps (non compris la queue). CEufs ellipsoïdaux, montrant 6 bandes méridiennes ayant l'apparence de côtes longitudinales, mais qui ne semblent pas présenter un relief appréciable, au moins chez les œufs encore dans l'utérus.

Le ơ est inconnu.

Dimensions, en $\mathrm{mm}$. :

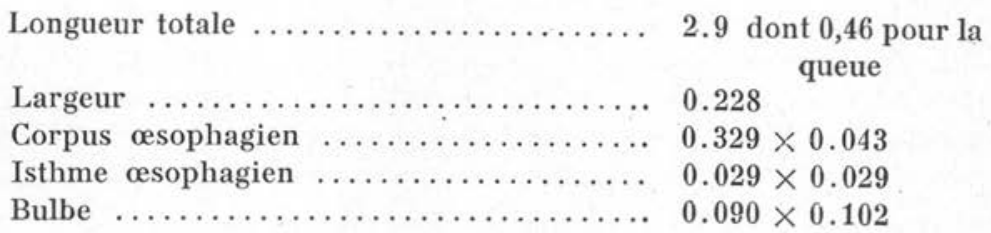


Distance de l'extrémité antérieure à l'anneau nerveux ................ 0.233

Distance de l'extrémité antérieure au pore excréteur ................... 0.595

Distance du pore excréteur à la vulve ... 0.836

Distance de la vulve à l'anus ........ 1.006

Cufs utérins, $92 \times 55-57 \mu, 95 \times 57-58 \mu, 100 \times 57 \mu$.

Provenance : intestin de Glomeris annulata Brandt, - Sète (Hérault) ; Odette Tuzet, leg., $1^{\mathrm{er}}-12-1950$.

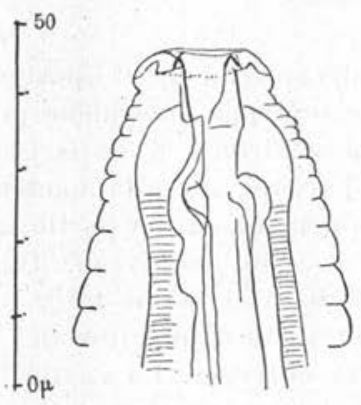

FIG. 3. - Thelastoma glomericola $\mathrm{n}$. sp.; intestin de Glomeris anrulata Brandt. Extrémité antérieure. Odette Tuzet leg., Sète (Hérault), 1-12-1950.

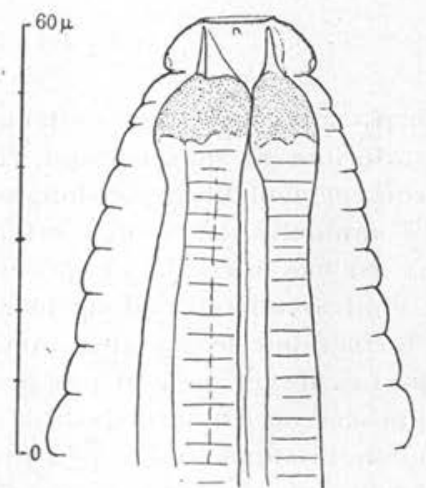

FiG. 4. - Même espèce et même provenance que pour la fig. 3. Extrémité antérieure d'un autre individu.

Discussion. - Par l'ornementation de ses œufs, l'espèce ci-dessus décrite est à comparer à $T h$. glomeridis (Linstow 1885) $=T h$. ovocostata (Linstow 1886), dont les œufs portent six côtes longitudinales ; mais, chez l'espèce de Linstow, les œufs sont sensiblement plus petits $(86 \times 43 \mu)$ et la queue est $1 / 10$ de la longueur du corps (3,7 mm.), donc proportionnellement beaucoup plus longue.

\section{THELASTOMA PTEROTON n. sp. (1)}

(Fig. 5-9, et pl. I, fig. 3)

6. Extrémité antérieure presque tronquée, sans lèvres saillantes. Stries cuticulaires transversales très prononcées, à peu près jusqu'à l'anus. Ailes latérales débutant à une petite distance de l'extrémité antérieure et s'étendant à peu près jusqu'à l'anus. Cavité buccale peu profonde, ne

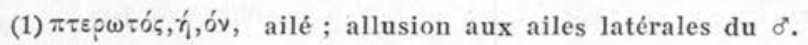


dépassant pas postérieurement le $2^{\circ}$ anneau. Gsophage subcylindrique, grêle, avec un très léger élargissement peu après son extrémité antérieure. Isthme court, bulbe gros, avec appareil masticateur; début de l'intestin un peu plus large que le bulbe. Pore excréteur au niveau du bulbe. Queue plutôt longue (approximativement $1 / 5^{\circ}$ ou $1 / 6^{\circ}$ de la longueur totale), brusquement rétrécie presque immédiatement après l'anus, gardant d'abord la même largeur dans environ la première moitié de

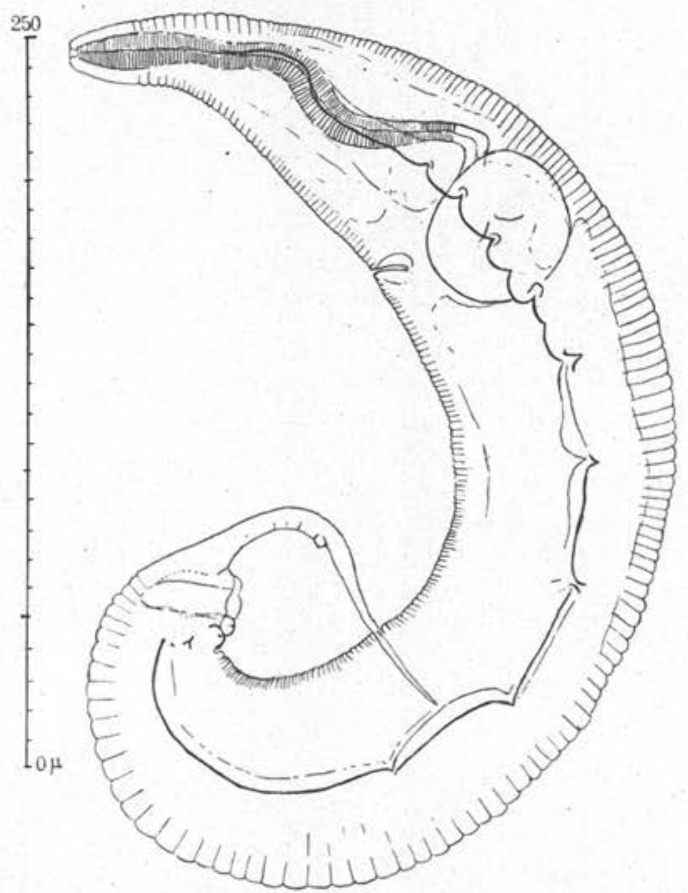

FIG. 5. - Thelastoma pteroton n. sp.; intestin de Julus (sensu lato) sp.; Daloa (Côte d'Ivoire). Individu $\delta^{\circ}$, vue latérale. Odette Tuzet leg., 1951 .

sa longueur, jusqu'à une paire de grosses papilles, puis devenant filiforme. Aucun spicule. Une paire de papilles préanales immédiatement avant l'orifice anal, une paire adanale, une paire immédiatement postanale et une $4^{\circ}$ paire, déjà mentionnée, à peu près à la mi-longueur de la queue.

Dimensions, en mm., d'un $\delta$ :

Longueur totale . . . . . . . . . . . 0.874

Plus grande largeur .............. 0.086

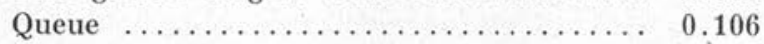

Ann. de Parasitologie, $\mathrm{T}$. XXVII, $\mathrm{N}^{\circ}$ 1-2-3. - 1952.

11. 
Corpus œsophagien + isthme, longueur $\ldots 0.170$

Distance de l'extrémité antérieure à l'anlargeur .... 0.014

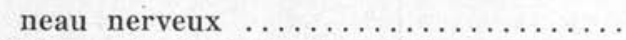

Distance de l'extrémité antérieure au pore

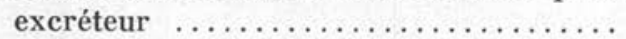

0.150

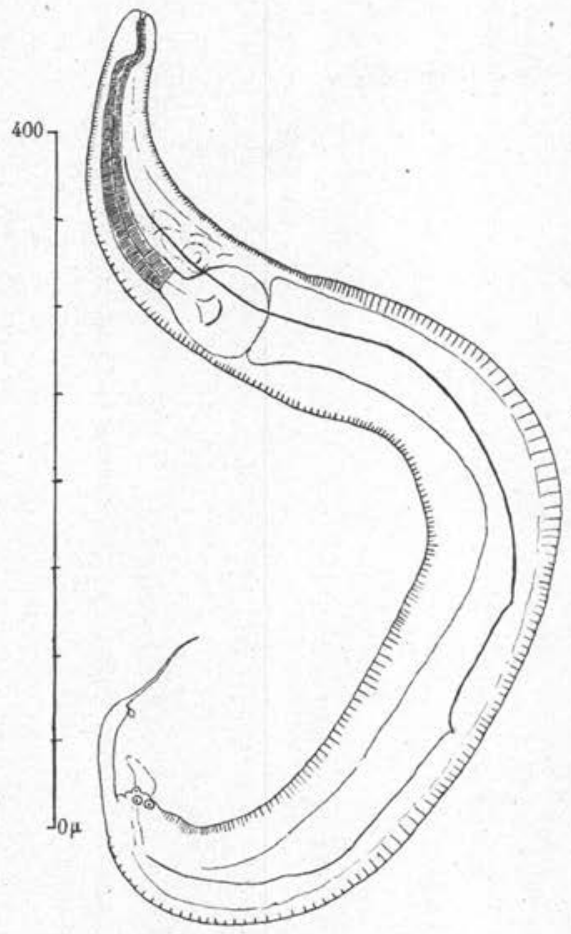

Fig. 6. - Mème espèce et même provenance que pour la fig. 5 . Individu ${ }^{\circ}$ de grande taille (env. $0 \mathrm{~mm} .9$ ).

․ Extrémité antérieure plutôt large, avec 4 papilles s'élevant un peu au-dessus du bord externe. Stries cuticulaires transversales délimitant des anneaux très accentués jusqu'à la queue. Cavité bucco-pharyngienne à peu près aussi profonde que large, atteignant le bord antérieur du $2^{\circ}$ anneau. Corpus œsophagien subcylindrique, se rétrécissant légèrement dans sa partie postérieure. Isthme court, en continuité avec le bulbe. Début de l'intestin plus large que le bulbe. Pore excréteur un peu antérieur à l'isthme œsophagien. Vulve à peu près à mi-distance du début de l'intestin et de l'anus. A une petite distance en arrière de l'anus, la queue se rétrécit, pour devenir un long prolongement filiforme. Il y a environ 30 ou 40 œufs utérins, à coque mince. 


\begin{tabular}{|c|c|}
\hline Distance de l'extrémité antérieure à la vulve & 0.90 \\
\hline Distance de la vulve à l'anus $\ldots \ldots \ldots \ldots$ & 0.442 \\
\hline$\ldots \ldots \ldots \ldots \ldots \ldots \ldots$ & 0.885 \\
\hline
\end{tabular}

Provenance : Intestin (juste en arrière des tubes de Malpighi), chez Julus (sensu lato) sp.; Daloa (Côte-d'Ivoire). Odette 'Tuzet, leg., 1951.

Discussion. - L'espèce ci-dessus décrite est à comparer avec celles dont le $\delta$ possède aussi, à l'état adulte, des ailes latérales ; le $\sigma$ de robustum a le pore excréteur plus postérieur et le prolongement caudal proportionnellement plus court; en outre, il a un spicule ; le ớ d'alatum (1) a le pore excréteur à peu près au même niveau que notre espèce de Daloa, mais ses ailes caudales sont localisées dans la région postérieure du corps et il a un spicule. Chez papilliferum, les ailes sont peu développées, atteignant seulement $4 \mu$ de large vers le milieu du corps, et il y a un spicule; néanmoins, notre espèce offre beaucoup de ressemblance avec papilliferum (=macramphidium), en particulier la forme de la queue de la $q$ est la même ( $c f$. J. R. Christie, 1931, p. 476, fig. 9 A).

\section{THELASTOMA BULHồsı (P.S. de Magalhães 1900)}

(Fig. 10 et pl. I, fig. 4)

Cette espèce a été décrite deux fois, d'abord par Magalhães (1900, p. 50-69, fig. 10-20, 23), ensuite par B. G. Chitwood (1932, p. 21, fig. 1-3), qui ont eu à leur disposition des individus des deux sexes, trouvés dans l'intestin de Periplaneta americana L. ; ceux de Magalhães avaient été récoltés à Rio-de-Janeiro ; ceux de Chitwood, vraisemblablement aux Etats-Unis. Je n'ai eu à ma disposition qu'une $\uparrow$, provenant d'un Myriapode du Brésil; cette $q$ présente quelques différences avec les descriptions publiées par Magalhães et Chitwood ; néanmoins, elle me paraît avoir des caractères qui, dans l'ensemble, concordent avec ceux de Bulhôesi. Le grand développement du $2^{\circ}$ anneau qui, chez mon exemplaire, atteint $18 \mu$ de long sur $40 \mu$ de large, avait été bien remarqué par Magalhães (1900, fig. 11 et 23). Quand Magalhães dit que l'œsophage ne possède pas de baguettes, je ne suis pas d'accord avec lui, car mon spécimen possède de fines baguettes œsophagiennes (2), et celles-ci présentent une légère constriction vers la limite entre les $3^{\circ}$ et $4^{\circ}$ cinquiè-

(1) Th. alatum Th. Johnston 1914 , a été trouvé dans une larve de Cétoine, Cacachroa decorticata Macl., à Cairns (North Queensland).

(2) Le terme " baguettes œsophagiennes " remplace le terme \& baguettes pharyngiennes 》 autrefois employé par O. Gares (1879, p. 316). 
Dimensions, en mm., d'une $q$ à maturité :

Longueur
Largeur

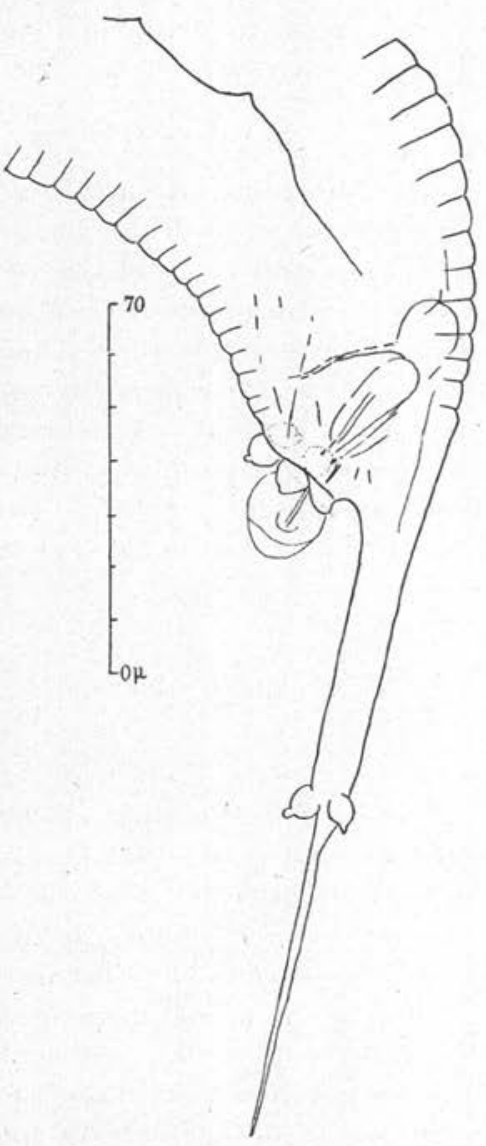

Fig. 7. - Même espèce et même provenance que pour les figures 5 et 6 . Extrémité postériure d'un $\delta^{*}$.
2.227

0.27

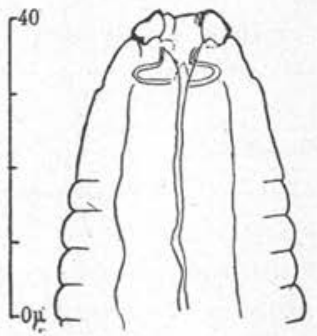

FIG. 8. - Même espèce et même provenance que pour les figures 5-7. Extrémité antérieure d'un $\sigma^{\circ}$ long d'environ $0 \mathrm{~mm}$. 75 , large de $0 \mathrm{~mm} .12$.

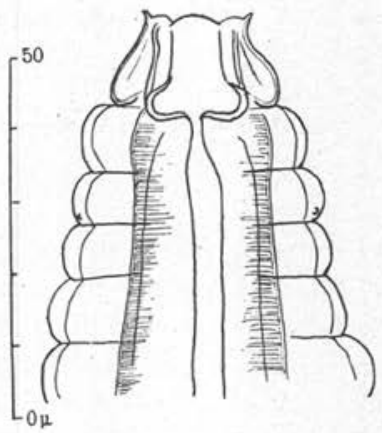

FIG. 9. - Même espèce et même provenance que pour les figures 5-8. Extrémité antérieure d'une $₹$ longue d'environ $2 \mathrm{~mm}$. 5 (dont 0,91 pour la queue), large d'environ $0 \mathrm{~mm}$. 288 . Distance jusqu'à la vulve $1 \mathrm{~mm} .12$; de la vulve à l'anus $0 \mathrm{~mm}$. 51 .

Corpus œsophagien, longueur ........ 0.38

$$
\text { largeur ......... } 0.030-0.033
$$

Isthme œsophagien, longueur $\ldots \ldots \ldots \ldots .0 .030$

largeur $\ldots \ldots \ldots \ldots \ldots$ 0.019

Distance de l'extrémité antérieure à l'anneau nerveux ................ 0.185 env.

Distance de l'extrémité antérieure au pore excréteur $\ldots \ldots \ldots \ldots \ldots \ldots \ldots \ldots \ldots \ldots \ldots \ldots \ldots$ 


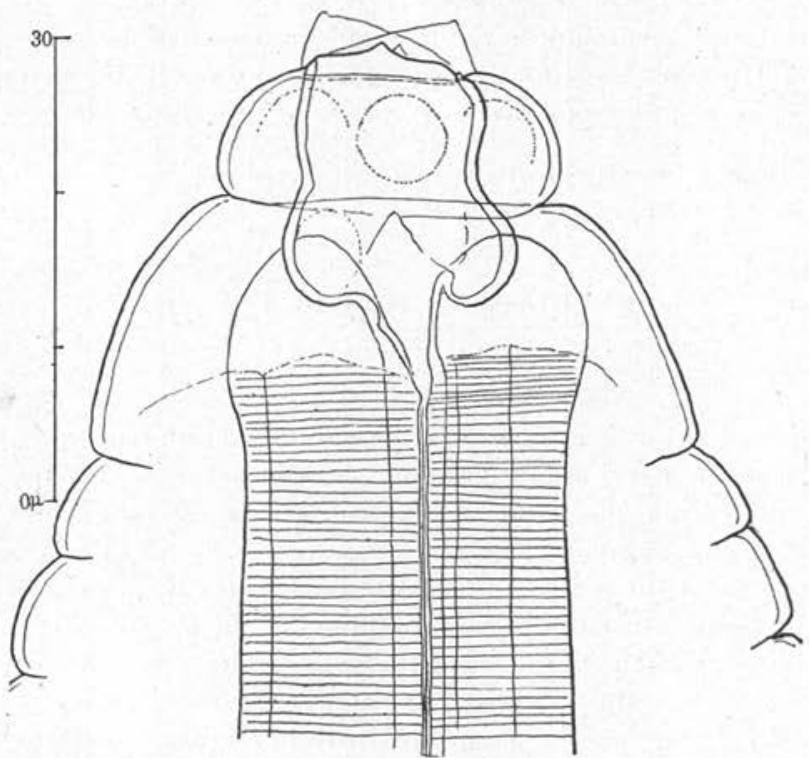

FiG. 10. - Thelastoma Bulhõesi (P.S. de Magalhães 1900); intestin de Gymnostreptus sp. Extrémité antérieure d'une ${ }^{\circ}$. Santa Adelia (Etat de São Paulo. Brésil), n 3.283, 7-1-1950. Odette Tuzet ded.

mes de leur longueur. Chitwood n'a rien dit des baguettes æesophagiennes.

\begin{tabular}{|c|c|c|c|}
\hline $\begin{array}{l}\text { Q DIMENSIONS D'APRÈS } \\
\text { EN MM. }\end{array}$ & MagalhaEs & Chitwoon & $\begin{array}{l}\text { MON } \\
\text { EXEMPLAIRE }\end{array}$ \\
\hline Longueur totale.$\ldots \ldots \ldots \ldots \ldots \ldots \ldots$ & $2,71(2,10-3,39)$ & $2,282,64$ & 2,78 \\
\hline Largeur... ........................ & $0,23-0,28(0,30)$ & $0,188-0,273$ & 0,255 \\
\hline 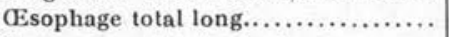 & - & $0.463-0,594$ & 0,535 \\
\hline Corpus esophagien long ............ & $0,36-0,40$ & $0,329-0,380$ & 0,505 \\
\hline$-\quad-\quad \operatorname{larg} \ldots \ldots \ldots \ldots$ & - & $0,038-0,040$ & 0,030 \\
\hline Isthme œsophagien long . ........... & - & $0,034-0,036$ & 0,030 \\
\hline$-\quad-\quad$ larg.............. & - & $0,022-0,030$ & 0,030 \\
\hline Bulbe $\ldots \ldots \ldots \ldots \ldots \ldots \ldots \ldots \ldots \ldots \ldots \ldots \ldots$ & - & $\begin{array}{r}0,086-0,106 \\
\times 0,080-0,095\end{array}$ & $\begin{array}{r}0,110 \\
\times 0,110\end{array}$ \\
\hline 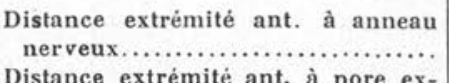 & - & env. 0,230 & 0,233 \\
\hline 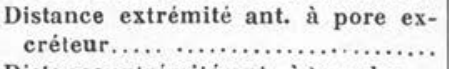 & 0,45 & env. 0,430 & 0,415 \\
\hline Distance exırémité ant. à la vulve... & - & $1,0-1,21$ & 1,315 \\
\hline Distance extrèmité ant. à l'anus..... & $1,70 \cdot 1,80$ & [env. $1,36-1,92]$ & 1,86 \\
\hline Queue.......................... & $0,78-0,81(0,82)$ & $0,720-0,920$ & 0,920 \\
\hline Gufs............................. & $0,080-0,085$ & $0,070-0,0 \leq 0$ & $0,066-0,069$ \\
\hline & $\times 0,060-0,067$ & $\times 0,050-0,072$ & $\times 0,052-\theta, 055$ \\
\hline
\end{tabular}


Mon spécimen paraissant avoir la région antérieure du corps en extension et étant un peu plus long que ceux de Chitwood, il est tout naturel que le corpus œsophagien soit aussi un peu plus long.

Provenance : Intestin de Gymnostreptus sp., de Santa Adelia (Etat de São-Paulo), $\mathrm{n}^{\circ} 3283 ; 7-1-1950$.

\section{SEVERIANOIA JULICOLA n. sp.}

(Fig. 11 et pl. I, fig. 5)

§. Corps subcylindrique, plus atténué antérieurement que postérieurement, distinctement annelé. Queue courte, cônique. Tête distincte, beaucoup plus large que haute, portant un feston à 8 lobes, non en relief. Cavité bucco-pharyngienne peu étendue, plus profonde que large. Corpus œsophagien grèle, fortement atténué antérieurement, s'élargissant postérieurement, se continuant par un isthme de même diamètre, rétréci à sa base, juste avant le bulbe. Intestin avec dilatation antérieure un peu plus large que le bulbe. Anneau nerveux en arrière de la mi-longueur de l'œsophage, un peu en avant de l'isthme. Pore excréteur loin en arrière du bulbe, à peu près aussi loin du bulbe que le bulbe est loin de la tête. Deux ovaires, appareil génital amphidelphe. Vulve non saillante, un peu après le $3^{\text {a }}$ cinquième de la longueur du corps, non compris la queue. CEufs ellipsoïdaux, au nombre d'environ 50 à 60 .

ơ inconnu.

Dimensions, en mm., de 2 :

\begin{tabular}{|c|c|c|}
\hline Longueur totale $\ldots \ldots \ldots \ldots \ldots$ & 2.7 & 2.9 \\
\hline Queue $\ldots \ldots \ldots \ldots \ldots \ldots \ldots$ & 0.158 & 0.160 \\
\hline Largeur $\ldots \ldots \ldots \ldots \ldots \ldots$ & 0.24 & 0.23 \\
\hline Corpus œesophagien $\ldots \ldots \ldots \ldots$ & $0.22 \times 0.040$ & $0.24 \times 0.035$ \\
\hline Isthme œsophagien $\ldots \ldots \ldots \ldots$ & $0.065 \times 0.040$ & $0.055 \times 0.035$ \\
\hline $\begin{array}{l}\text { Rétrécissement prébulbaire, lar- } \\
\text { geur } \ldots \ldots \ldots \ldots \ldots \ldots \ldots \ldots \ldots\end{array}$ & 0.025 & 0.030 \\
\hline Bulbe $\ldots \ldots \ldots \ldots \ldots \ldots \ldots$ & $0.105 \times 0.115$ & $0.100 \times 0.115$ \\
\hline Distance de l'extrémité antérieure & & \\
\hline à l'anneau nerveux $\ldots \ldots \ldots$. & 0.197 & 0.202 \\
\hline $\begin{array}{l}\text { Distance de l'extrémité antérieure } \\
\text { au pore excréteur } \ldots \ldots \ldots \ldots\end{array}$ & 0.60 & 0.68 \\
\hline $\begin{array}{c}\text { Distance du pore excréteur à la } \\
\text { vulve } \ldots \ldots \ldots \ldots \ldots \ldots \ldots \ldots \ldots \ldots \ldots\end{array}$ & 1.10 & 1.00 \\
\hline Distance de la vulve à l'anus ... & 0.80 & 1.02 \\
\hline
\end{tabular}

CEufs $(\mathrm{en} \mu), 92.4 \times 57.8$.

Provenance : Intestin de Julus terrestris L. ; Banyuls-sur-Mer (Pyrénées-Orientales) ; Jean Théodoridès, leg., mai 1950. 


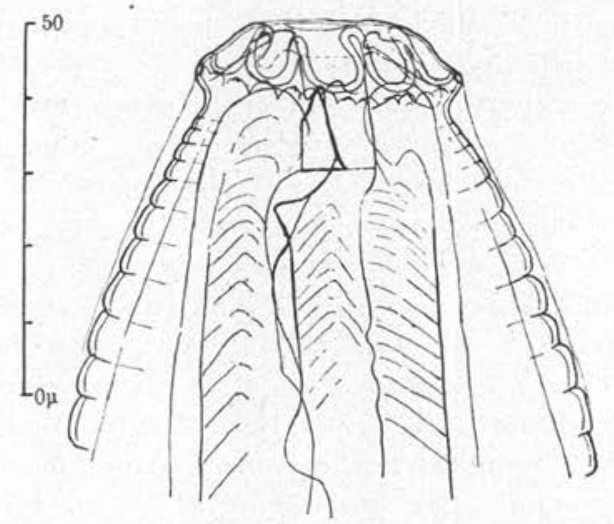

FIG. 11. - Severianoia julicola n. sp. ; intestin de Julus terrestris L. Extrémité antérieure d'une . Jean Théodoridès leg., mai 1950. Banyuls-sur-Mer (Pyrénées-Orientales).

Discussion. - Dans le genre Thelastoma, la queue de la $q$ est filiforme ou finement atténuée — subfiliforme ; l'espèce ci-dessus n'appartient donc pas à ce genre ; il est vraisemblable qu'elle appartient au genre Severianoia, où la queue est plus courte que chez Thelastoma et conique ou presque conique, non filiforme. En attendant de pouvoir examiner de nouveaux matériaux (je n'ai eu à ma disposition que deux $q$ en médiocre état), j'attribue l'espèce de Banyuls, provisoirement, à Severianoia.

\section{Famille RHIGONEMATIDAE (P. Artigas 1930)}

\section{Sous-famille RHIGONEMATINAE (P. Artigas 1930)}

Rhigonema (N. A. Cobb 1898)

La diagnose de ce genre a été donnée par J. R. Christie et N. A. Cobb (1927, p. 18-19). Dans la même publication, J. R. Christie et N. A. Cobb ont montré que l'emploi du genre Isakis Ch. Lespès $\mathbf{1 8 5 6}$ pour des espèces présentant les caractères génériques de Rhigonema n'était pas acceptable et devait être abandonné ; dans Rhigonema, ils admirent, outre brevicolle N. A. Cobb 1898 (générotype), les espèces infecta (J. Leidy 1849), acuminata (J. d'Udekem 1859), multipapillata (K. I. Skrjabin 1916), et, dubitativement, « Isacis modiglianii 》 C. Parona 1896.

Avant la publication de J. R. Christie et N. A. Cobb (communi- 
cation présentée le 27 novembre 1926, parue le 3 janvier 1927), il aurait été bien difficile de placer ces espèces dans Rhigonema, car aucune diagnose générique n'avait été proposée par N. A. Cobb, et les figures du générotype données par N. A. Cobb (1898, p. 295, fig. $29 \mathrm{I}-29 \mathrm{IV}$ ), très petites, n'étaient pas suffisantes (en particulier en ce qui concerne l'appareil génital †) pour que l'on puisse étendre l'acception de Rhigonema à ces espèces.

Dans une publication portant la date du 24 décembre 1926, P. Artigas décrivit : Isakis falcatum P. Artigas, Isakis subulatum P. Artigas et Rhigonema truncatum P. Artigas, conservant Isakis (sensu K. I. Skrjabin 1916 et sensu H. A. Baylis et R. Daubney, non Lespès 1856) et le considérant comme distinct de Rhigonema, en raison de la présence, chez Rhigonema, d'un sac impair (qu'il appela alors vésicule séminale), annexé à la vulve, sac n'existant pas chez celles des espèces qu'il attribua alors à Isakis.

Le rejet par R. J. Christie et N. A. Cobb de l'emploi d'Isakis pour des espèces à caractères conformes à la diagnose de Rhigonema ne fut pas tout de suite accepté par P. Artigas, et celui-ci, dans sa thèse (1929, p. 96-97), conserva Isakis à côté de Rhigonema, laissant dans Isakis les espèces falcatum et subulatum, dont les $q$ sont dépourvues du sac impair annexé à la vulve, considéré par lui comme caractère générique de Rhigonema. Il est donc surprenant que, parmi les espèces qu'il admit alors dans Isakis, Artigas (1929, p. 97) ait cité, entre autres espèces, celle décrite par J. Leidy sous le nom d'Ascaria infecta J. Leidy, alors que J. Leidy (1853, pl. VII, fig. 14) avait donné une bonne figure montrant clairement ce sac impair annexé à la vulve, considéré comme caractéristique et distinctif de Rhigonema par Artigas lui-même.

C'est seulement en 1930 que P. Artigas abandonna Isakis (sensu Skrjabin et sensu Baylis et Daubney) pour les espèces répondant à la diagnose générique de Rhigonema, mais ił proposa alors Dudekemia pour séparer de Rhigonema celles de ces espèces dépourvues de ce sac impair annexé à la vulve. Dans Dudekemia, furent placés par Artigas (1930, p. 22) : acuminata (J. d'Udekem 1859), multipapillata (K. I. Skrjabin 1916), falcata (P. Artigas 1926), subulata (P. Artigas 1926), robusta (A. C. Walton 1928), et trois espèces nouvelles : multispinosa P. Artigas 1930, brevicaudata P. Artigas 1930 et simile P. Artigas 1930. Dans Rhigonema, trois espèces : brevicolle N. A. Cobb 1898 (générotype), infecta (J. Leidy 1849) et truncata P. Artigas 1926, furent conservées par Artigas.

Ce sac impair, annexé antérieurement à la vulve, que J. Leidy (1849, p. 230) avait considéré comme « seminal receptacle », puis (1853, p. 44, 64) comme spermathèque, fut appelé par P. Artigas 
d'abord (1926, p. 97,$102 ; 1929$, p. $96,105,106)$ « vesicula seminal », ensuite $(1930$, p. 21) spermathèque; il ne doit pas être confondu avec la dilatation piriforme présente entre l'ovaire et l'utérus, qui est généralement appelée spermathèque par les descripteurs et n'a rien de commun avec le sac impair en question. Celui-ci a été désigné sous les noms de "large unpaired bulb-like structure » et de « syringate-bulb», par L. J. Thomas (1930, p. 33, 34 , pl. III, fig. 11).

Dans leur diagnose de Rhigonema, J. R. Christie et N. A. Cobb (1927, p. 19) ont qualifié de spermathèque une dilatation située entre l'ovaire et l'utérus ; en ce qui concerne le sac impair, ils ont dit ceci : chez les jeunes femelles à maturité, un « impaired enlargement » est parfois présent près de la vulve et des spermatozoïdes peuvent s'y rencontrer. Pour Christie et Cobb, ce sac impair est donc tantôt présent, tantôt absent chez Rhigonema; sa présence n'est donc pas constante dans le genre; il n'a été ni signalé, ni représenté par N. A. Cobb (1898, fig. 29 III) pour le générotype ; on peut done se demander si son absence peut justifier un genre particulier ; de plus, chez les espèces où il est dit exister, il n'est pas toujours bien nettement observable chez tous les individus; dans beaucoup de cas, on peut douter de sa présence; je crois donc que Dudekemia ne peut avoir, tout au plus, que la valeur d'un sousgenre de Rhigonema.

B. G. Chitwood et M. B. Chitwood (1940, p. 149, fig. $116 \mathrm{~J}$ et $119 \mathrm{D} ; 1950$, p. 147 , fig. $116 \mathrm{~J}$ et $119 \mathrm{D})$ ont donné une figure détaillée de l'appareil $q$ de $R$. infectum (J. Leidy) (1), montrant les connections du sac impair; ils disent que, chez cette espèce, la « vagina vera " est en connection avec un " massive sac 》 et, par celui-ci, avec l'utérus amphidelphe ; ils rappellent que, ce sac étant absent chez d'autres espèces, Artigas a formé pour celles-ci le genre Dudekemia ; ils indiquent que, chez infectum, l'extrémité ovarienne de chaque utérus étant différenciée en spermathèque et chambre de fécondation, ils présument que la grande chambre connectée avec la vulve est une poche à œufs; ils ne s'accordent donc pas avec Artigas qui l'a considérée comme spermathèque.

Les espèces décrites de Rhigonema (incl. Dudekemia) sont, par ordre chronologique :

(1) Pour l'anatomie de Rhigonema infectum (J. Leidy), qui est l'espece la mieux connue du genre, on se reportera à B. G. Chitwood et M. B. Chitwood (1937, fig. $11 \mathrm{E}-\mathrm{F}$; 1938, fig. $57 \mathrm{I}, 103 \mathrm{~A}$; 1940 , fig. $116 \mathrm{~J}, 118 \mathrm{C}, 119 \mathrm{D}, 124 \mathrm{E}$, $126 \mathrm{G}$; 1950 , fig. $11 \mathrm{E}-\mathrm{F}, 57 \mathrm{~J}, 103 \mathrm{~A}, 116 \mathrm{~J}, 118 \mathrm{C}, 119 \mathrm{D}, 124 \mathrm{E}, 126 \mathrm{G})$. 

R. brevicolle N. A. Cobb 1898, générotype.
R. infectum (J. Leidy 1849).
R. (D.) acuminatum (J. d'Udekem 1859).
R. (D.) multipapillatum (K. I. Skrjabin 1916).
R. (D.) falcatum (P. Artigas 1926).
R. (D.), subulatum (P. Artigas 1926).
R. truncatum P. Artigas 1926.
R. (D.) multispinosum (P. Artigas 1930).
R. (D.) brevicaudatum (P. Artigas 1930).
R. (D.) simile (P. Artigas 1930).
R. nigella L.-J. Thomas 1930.

Peut-être devra-t-on ajouter, après réexamination des types de la collection Corrado Parona : $R$. (D.) modiglianii (C. Parona 1896) et R. (D.) silvestrii (C. Parona 1896) (1).

En ce qui concerne $R$. (D.) robustum A. C. Walton 1928, de la collection J. Leidy, il semble, d'après ce qui a été dit des spécimenstypes, parasites de Myriapodes, de la collection J. Leidy, par B. G. Chitwood (1932, p. 15) et J. R. Christie (1938, p. 67), que ce soit $R$. infectum (J. Leidy), bien que A. C. Walton (1928, p. 94) ait d'avance rejeté cette possibilité et bien que A. C. Walton (1928, pl. V, fig. 25) n'ait pas figuré le sac impair annexé à la vulve, mentionné et figuré par J. Leidy chez infectum.

Les espèces de Rhigonema (incl. Dudekemia) se distinguent entre elles, principalement par les caractères suivants :

a) Présence ou absence de pilosité sur la partie antérieure du corps. (Cette pilosité existe seulement, d'après les descriptions, chez brevicaudatum, multispinosum, nigella; d'après une figure d'infectum, donnée par B. G. et M. B. Chitwood [1938, fig. 57 I et 1950, fig. 57 I], elle existe aussi chez cette espèce).

b) Présence ou absence de stries transversales de la cuticule. (Ces stries sont complètement absentes chez multipapillatum, d'après K. I. Skrjabin, 1916, p. 78, 143).

c) Longueur de la partie antérieure, par rapport à la longueur de la partie postérieure, des 6 «baguettes œsophagiennes 》 contenues dans le manchon musculaire œsophagien. (Ces deux parties semblent être d'égale longueur chez multipapillatum, d'après la figure donnée par K. I. Skrjabin, 1916, pl. VIII, fig. 60. La partie antérieure est plus longue que la postérieure chez nigella, d'après L.-J. Thomas, 1930, pl. III, fig. 1).

(1) Si l'examen du type d'Isacis Silvestrii C. Parona, générotype d'Haplacis A. Railliet et A. Henry (1916, p. 114), venait à montrer que cette espèce est bien un Rhigonema, le genre Haplacis tomberait en synonymie. 
d) Présence ou absence du sac impair annexé antérieurement à la vulve. (La présence de ce sac a été reconnue chez infectum, truncatum, nigella).

e) Forme et taille des spicules.

f) Longueur de la queue dans les deux sexes.

g) Emplacement de la vulve plus ou moins loin en arrière de la milongueur.

h) Nombre et disposition des papilles caudales.

\section{RHIGONEMA SUBTRUNCATUM n. sp.}

(Fig. 12-15)

La pilosité cuticulaire, très fine et très serrée, disparaît peu après le niveau du début de l'intestin. La partie antérieure des « baguettes pharyngiennes » est aussi longue, ou presque, que la postérieure. Le sac prévulvaire (spermathèque de J. Leidy 1853 , et de P. Artigas, $1930=$ "syringate-bulb » de L. J. Thomas 1930) est bien apparent. La queue se rétrécit très brusquement en formant une pointe courte.

Pour une $\varsubsetneqq$ longue de $4 \mathrm{~mm}$. 825, large de $0,30-0,32$, la vulve est située à une distance de $2,8 \mathrm{~mm}$. de l'extrémité antérieure, et 2,025 de l'extrémité postérieure ; la queue est longue de 0,120 . Chez cette $q$, j'ai compté 41 œufs utérins, mesurant $95-96 \times 72-74 \mu$, avec une coque épaisse d'environ $3 \mu$. Chez une autre $q$, les œufs utérins mesurent $90 \times 72,95 \times 72$, $98 \times 66,101 \times 66 \mu$, et sont au nombre de 35 . Chez une $q$ immature, longue de $3,9 \mathrm{~mm}$., large de 0,30 , la partie postvulvaire est longue de 1,55 , dont 0,140 pour la queue.

Les spicules sont robustes, à structure réticulée, ou d'apparence spongieuse. Chez un spécimen long de $3,6 \mathrm{~mm}$., large de 0,2 , ils sont longs d'environ 0,350 .

Provenance : $a$ ) Intestin de Gymnostreptus sp., de Santa Adelia (Etat de São-Paulo), 7-1-1950; $\mathrm{n}^{\circ} 3283$.

b) Intestin d'Hemigymnostreptus sp., de Santa Adelia (Etat de SãoPaulo) ; 7-1-1951; $\mathrm{n}^{\circ} 3281$.

Discussion. - Cette espèce présente la plus grande ressemblance avec $R$. brevicaudatum (P. Artigas 1930), qui a été placé dans Dudekemia par Artigas ; mais, chez brevicaudatum, il n'y a pas de sac prévulvaire, les spicules sont moins larges, et la partie antérieure des «baguettes pharyngiennes » est plus courte que la postérieure (d'après la fig. 15, pl. X, de P. Artigas, 1930). 

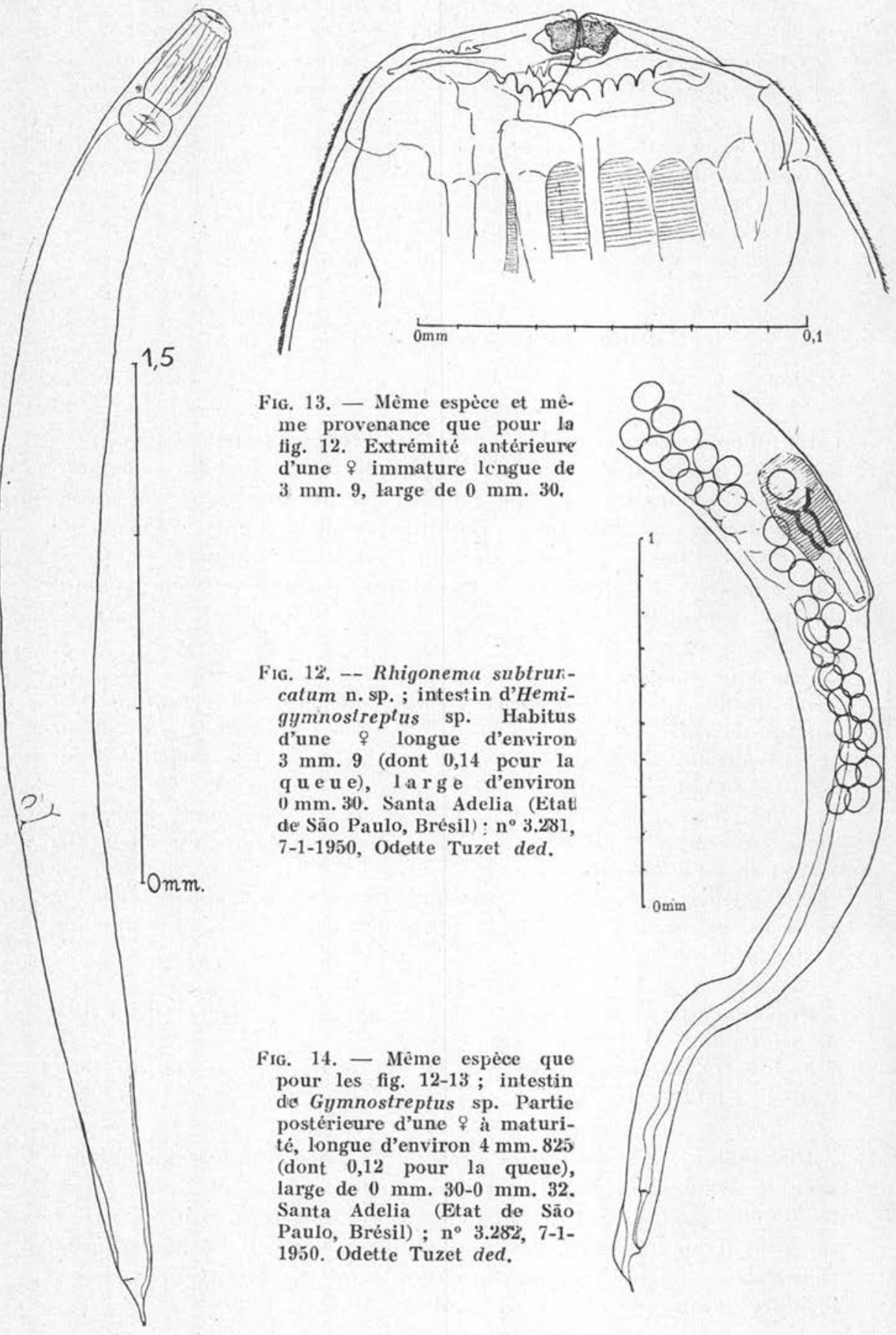

FIG. 13. - Mème espèce et mê. me provenance que pour la fig. 12. Extrémité antérieure d'une $\$$ immature lcngue de $3 \mathrm{~mm}$. 9, large de $0 \mathrm{~mm}$. 30 .

Fig. 12', - Rhigonema subtrurcatum n. sp. ; intestin d'Hemigyminostreptus sp. Habitus d'une $q$ longue d'environ $3 \mathrm{~mm} .9$ (dont 0,14 pour la queue), l a rge d'environ $0 \mathrm{~mm}$. 30. Santa Adelia (Etat de Sảo Paulo, Brésil) : $\mathbf{n}^{\circ} 3.281$, 7-1-1950, Odette Tuzet ded.

FIG. 14. - Même espèce que pour les fig. 12-13; intestin dis Gymnostreptus sp. Partie postérieure d'une ơ à maturité, longue d'environ $4 \mathrm{~mm}$. 825 (dont 0,12 pour la queue), large de $0 \mathrm{~mm}$. $30-0 \mathrm{~mm}$. 32 . Santa Adelia (Etat de São Paulo, Brésil) ; ${ }^{\circ} 3.282$, 7-11950. Odette Tuzet ded.

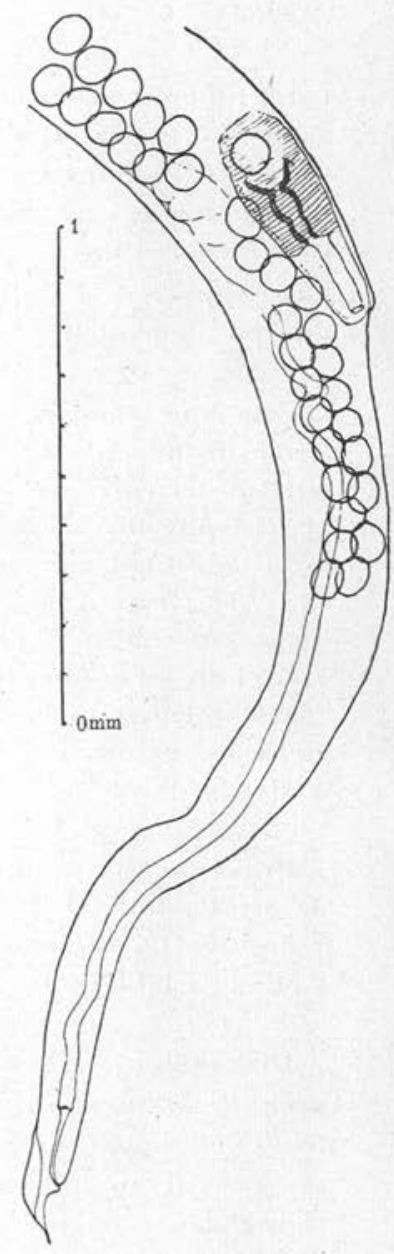


Rhigonema truncatum P. Artigas est extrêmement voisin, mais sa cuticule n'est pas pileuse ; si Artigas avait observé une pilosité cuticulaire chez truncatum, j'aurais rapporté mes spécimens à cette espèce, malgré les dimensions un peu plus grandes de leurs œufs.

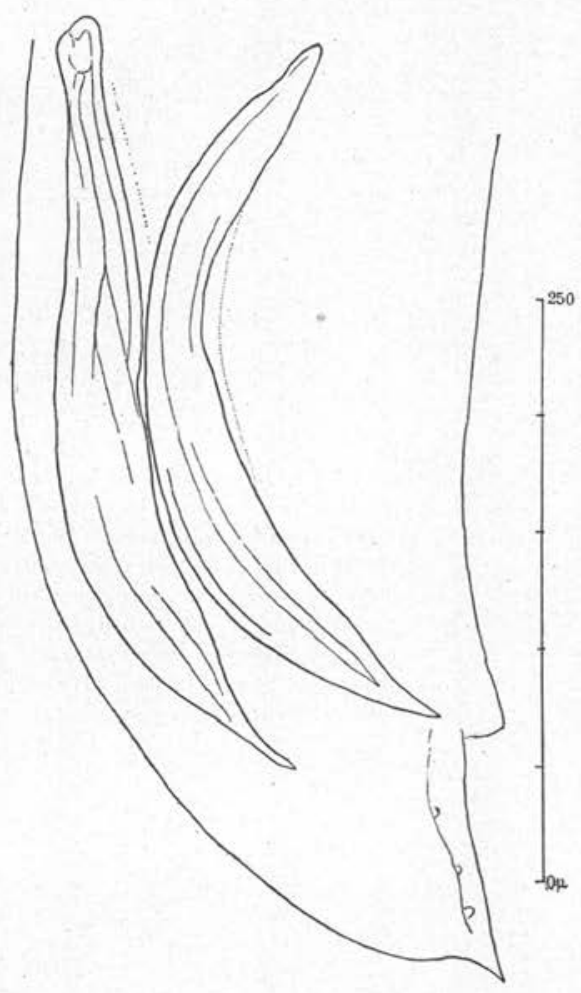

FIG. 15. - Même espèce et même provenance que pour la figure 14. Partie postérieure d'un of long d'environ $3 \mathrm{~mm} .6$ (dont environ 0,108 pcur la queue), large d'env. $0 \mathrm{~mm} .2$.

\section{RHIGONEMA LONGICAUDATUM n. sp.}

(Fig. 16-21)

Cette espèce est très voisine des autres espèces à cuticule antérieurement pileuse, mais aucune de celles-ci ne présente une queue aussi longue et aussi effilée chez la ๆ. La pilosité cuticulaire disparaît vers le début de l'intestin. La partie antérieure des "baguettes œsophagiennes » est densément striée transversalement et occupe beaucoup moins de la moitié de la longueur; la partie inférieure est légèrement arquée et s'atténue en pointe. Le sac prévulvaire est très développé. Le nombre des œufs utérins varie de 34 à $45 \mathrm{chez}$ le plus grand nombre des $q$, mais parfois il atteint $80-84$. 
Chez une $q$ à maturité, longue de $3,8 \mathrm{~mm}$., large de 0,20 , la partie prévulvaire est longue de 2,15 ; la queue effilée est longue de 0,30 , les œufs utérins mesurent $69 \times 55,81 \times 66,83 \times 69,89 \times 71 \mu$, avec une coque dont l'épaisseur augmente d'environ $3 \mu$ à environ $5,7 \mu$. Chez une autre $q$ à maturité, longue de $3,58 \mathrm{~mm}$., large de $0,215-0,230$, la partie prévulvaire

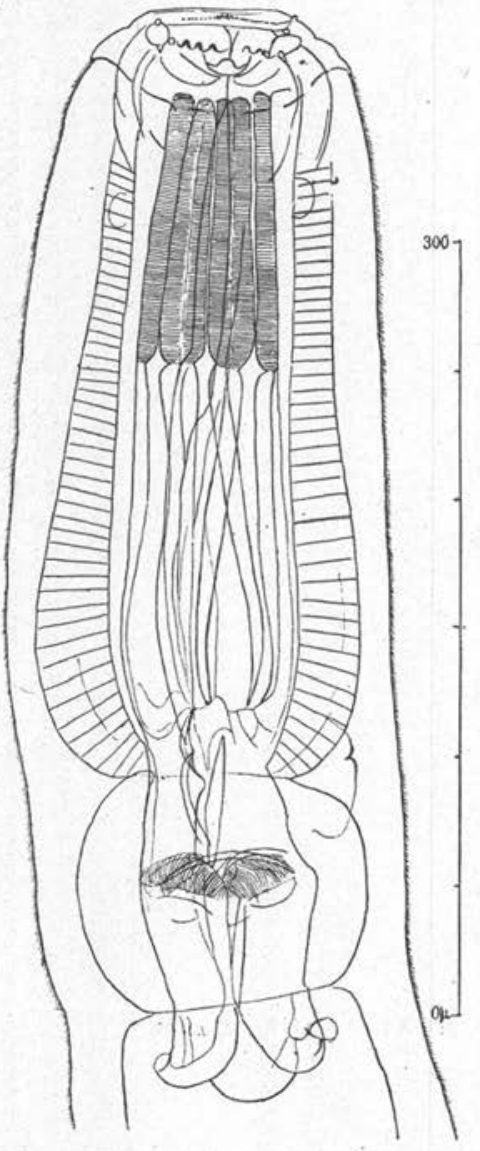

FIG. 16. - Rhigonema longicalldatum n. sp., de l'intestin de Leptodesmus paulistus $\mathrm{H}$. W. Brölemann. Région antérieure d'une of immature longue d'environ $3 \mathrm{~mm} .26$ (dont 0,250 pour la queue). San Sebastino (Etat de São Paulo, Brésil). Otto Schubart leg.

est longue de 1,884 , la partie postvulvaire de 1,696 , dont 0,307 pour la queue, les œufs mesurent $83 \times 66$, à $86 \times 63 \mu$; la coque atteint un maximum de $5,7 \mu$ environ. Une $q$ avait des œufs ne dépassant pas $75 \times 60$ à $78 \times 63 \mu$. Chez une immature, longue de 3,26 , large de $0,15-0,17$, la partie prévulvaire est longue de 1,74 , la partie postvulvaire de 1,52 (dont 0,250 pour la queue). Chez une autre immature, longue de $2,27 \mathrm{~mm}$., avec une largeur de 0,13-0,15, la queue est longue de 0,20.

Les spicules sont très robustes ; chez un $\sigma^{*}$, long de $3 \mathrm{~mm}$. (dont 0,179 


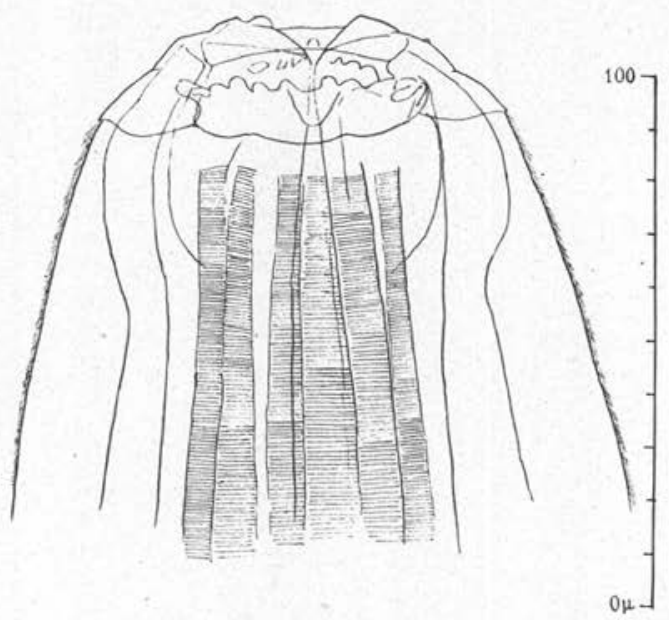

Fig. 17. - Mème espèce et même provenance que pour la figure 16. Extrémité antérieure d'une $\uparrow$ immature longue d'environ $2 \mathrm{~mm}$. 27 (dont 0,20 pour la queue), large de $0 \mathrm{~mm} .13-0 \mathrm{~mm} .15$.

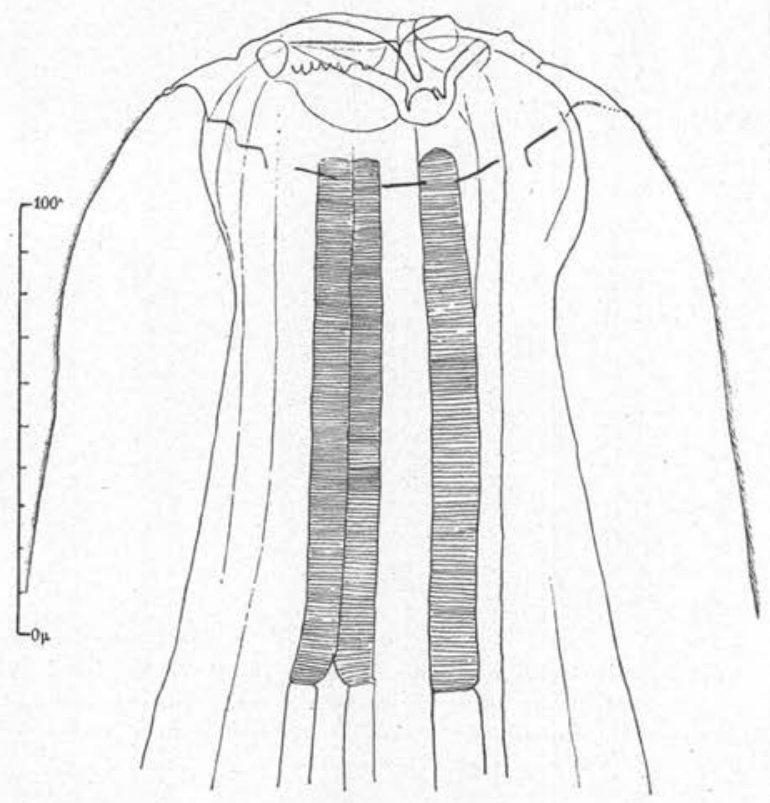

Fig. 18. - Même espèce et même provenance que pour les fig. 16-17. Région antérieure d'une $\Varangle$ à maturité longue d'environ $3 \mathrm{~mm} .8$ (dont 0,30 pour la queue), large de $0 \mathrm{~mm} .20$. 

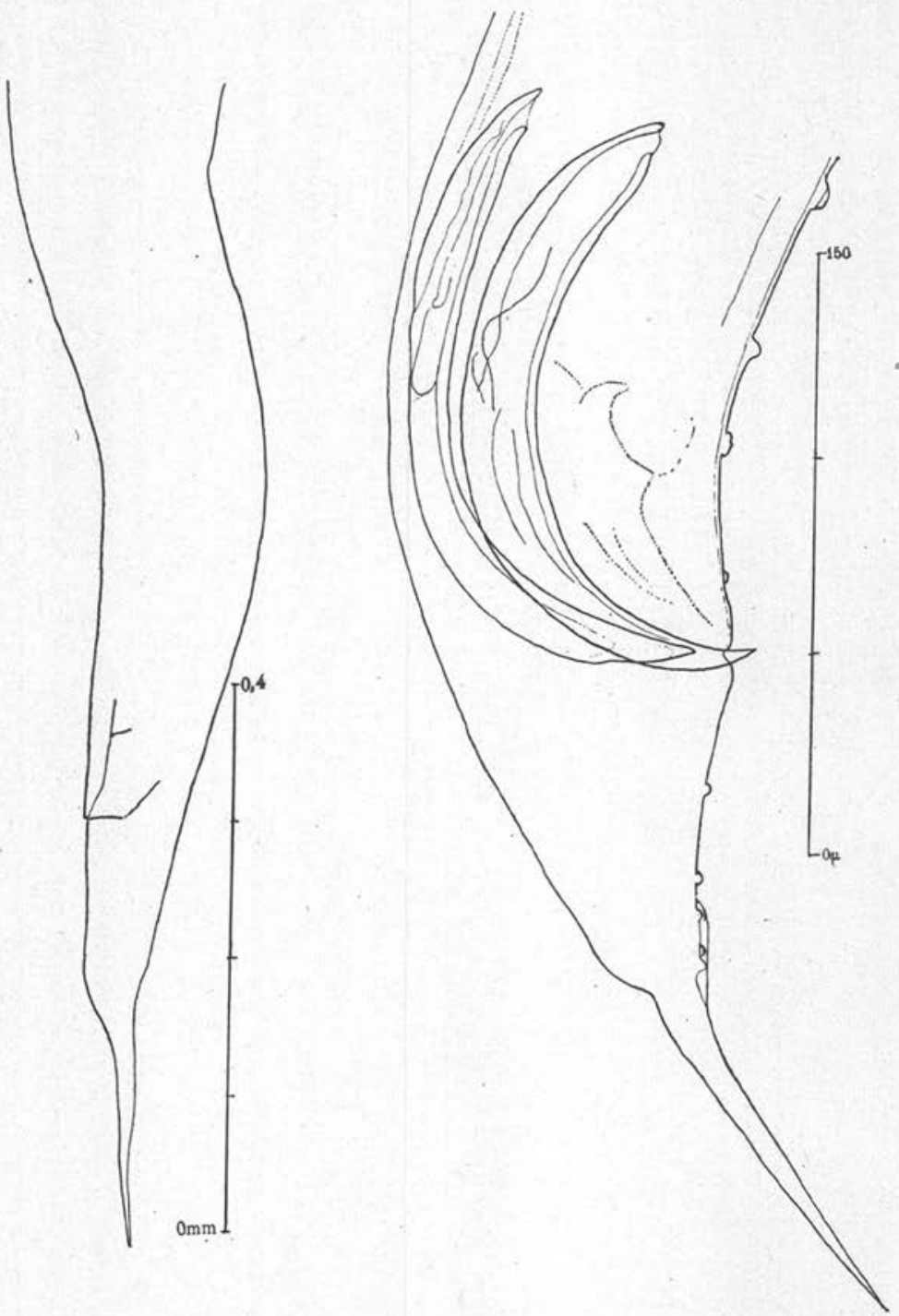

Fig. 19. - Même espèce que pour les figures 16-18. Intestin de Leptodesmus jucundus H. W. Brölemann. Brésil. Odette Tuzet ded. Habitus de la région postérieure d'une $q$ à maturité, longue d'environ $3 \mathrm{~mm}$. 58 (dont 0,307 pour la queue), large d'environ $0 \mathrm{~mm} .215-0 \mathrm{~mm} .230$.

Fic. 20. - Même espèce et même provenance que pour la fig. 19. Région postérieure d'un $\sigma^{*}$ long d'environ $3 \mathrm{~mm}$. (dont 0,179 pour la queue), large d'environ $0 \mathrm{~mm} .18$. 


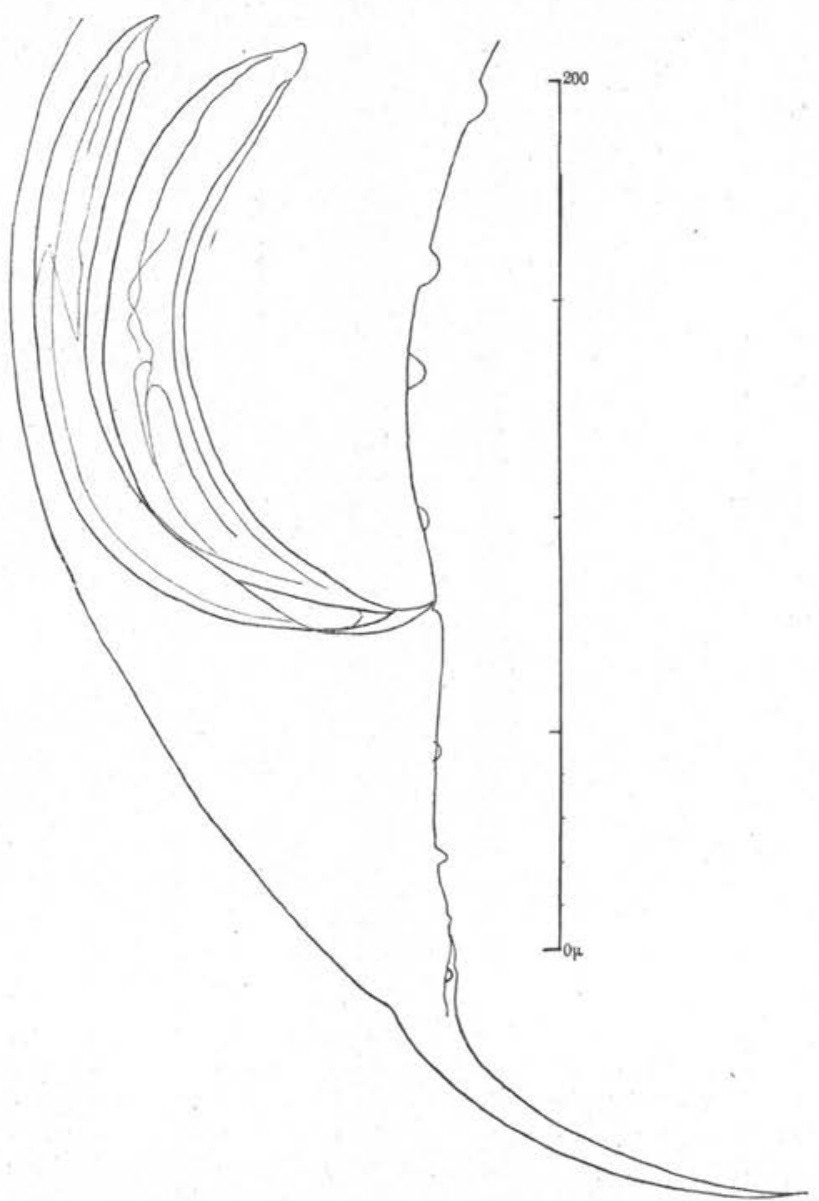

Fig. 21. - Même espèce et mème provenance que pour les fig. 19-20. Région postérieure d'un $\sigma$ long d'environ $2 \mathrm{~mm} .9$ (dont 0,196 pour la queue), large d'environ $0 \mathrm{~mm}$. 19. La queue est proportionnellement plus longue chez cet individu que chez celui de la fig. 20.

pour la queue), large de 0,18 , ils sont longs de 0,185 ; chez un autre $\delta$, long de $2,9 \mathrm{~mm}$. (dont 0,196 pour la queue), large de 0,19 , ils sont longs de $0,200(0,19-0,21)$.

Provenance : a) Intestin de Leptodesmus paulistus H. W. Brölemann, q, de San-Sébastino (Etat de São-Paulo). Otto Schubart, leg.

b) Intestin de Leptodesmus jucundus W. H. Brölemann, Brésil.

Ans. de Parasitologie, T. XXVII, $\mathrm{N}^{\circ} 1-2-3 .-1952$.

12. 

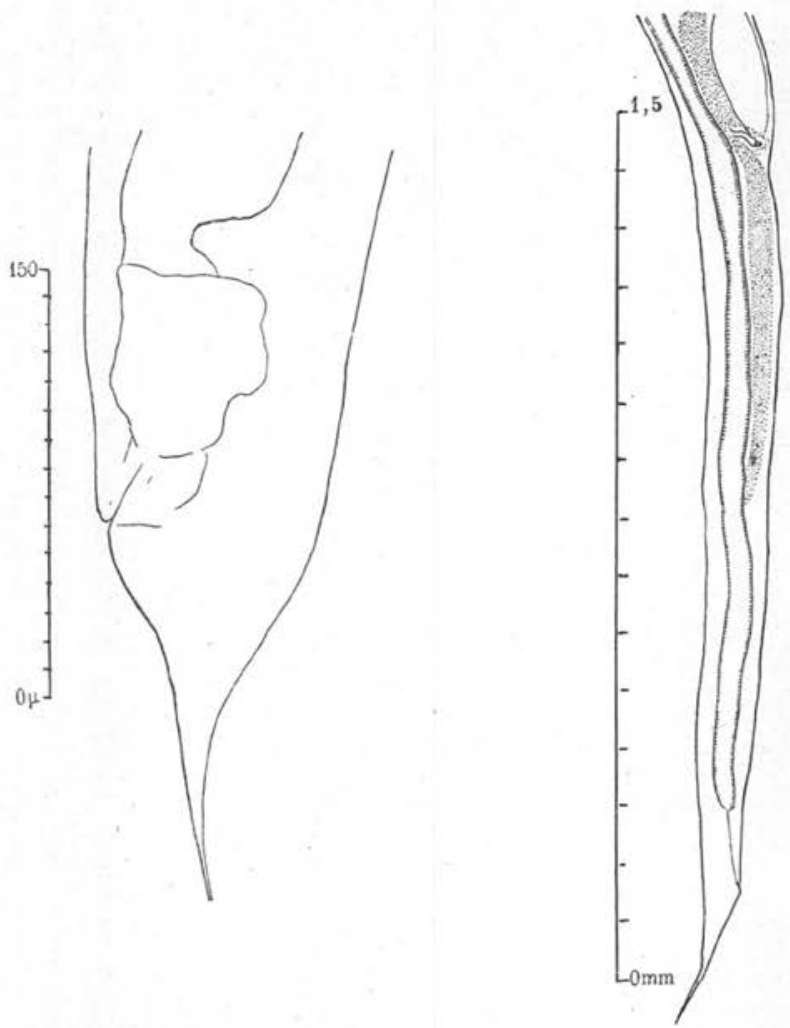

FiG. 22. - Rhigonema multispinosum (P. Artigas 1930). Intestin de Rhinocricus cachoeirensis O. Schubart. (Etat de São Paulo, Brésil), 1900. Odette Tuzet ded.

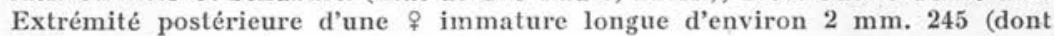
0,13 pour la queue).

F1G. 23. - Même espèce que pour la fig. 22. Intestin de Leptodesmus paulistus H. W. Brölemann, ๆ ; São Sebastino (Etat de São Paulo, Brésil). O. Schubart leg. Région postérieure d'une $\subsetneq$ immature longue d'environ $3 \mathrm{~mm}$. 26 .

\section{RHigonemA MULTISPINOSUM (P. Artigas 1930)}

(Fig. 22-26)

A cette espèce, je rapporte, avec beaucoup d'hésitation, quelques spécimens de Rhigonema à tégument pileux antérieurement, ne me semblant pas identifiables aux autres espèces présentant cette pilosité, c'est-à-dire à brevicaudatum, nigella, longicaudatum et subtruncatum. Chez ces spécimens, le prolongement caudal est nettement plus long que chez brevicaudatum. Les $q$ que j'ai examinées sont immatures et ne montrent pas de sac prévulvaire. 
Pour une $q$ immature, longue de $2,27 \mathrm{~mm}$., large de 0,15 , la queue est longue de 0,20 ; pour une autre, longue de $3,26 \mathrm{~mm}$., la longueur jusqu'à la vulve est de 1,740 et, après la vulve, 1,520 (dont 0,249 pour la queue); pour une troisième, longue de $2,245 \mathrm{~mm}$., la queue est longue de 0,13 .

Les $\delta$ ont des spicules plutôt robustes, plus longs que la queue. Pour un $\sigma^{2}$, long de $3,136 \mathrm{~mm}$., large de 0,17 , les spicules sont longs de

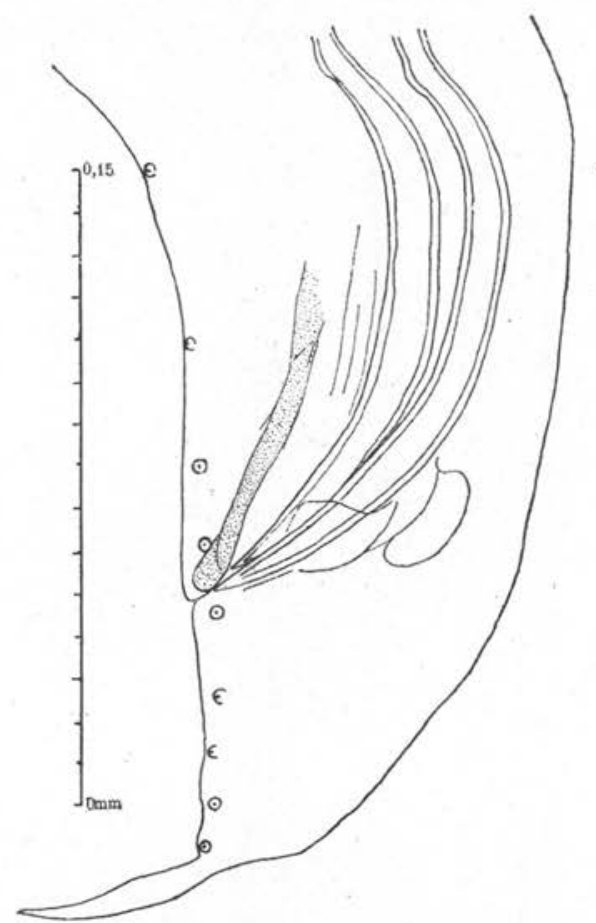

FIG. 24. - Même espèce et mème provenance que pour la figure 23. Région postérieure d'un ơ long d'environ $3 \mathrm{~mm}$. 136 (dont 0,107 pour la queue), large de $0 \mathrm{~mm}$. 17. Les spicules sont longs de $0 \mathrm{~mm} .140-0 \mathrm{~mm} .145$.

0,140-0,145 et la queue de 0,107. Pour un autre $\delta$, long de $2,65 \mathrm{~mm}$., large de 0,12 , les spicules sont longs de 0,183 et la queue de 0,081 . Les spicules ont une structure réticulée très apparente (fig. 26).

J'ai compté 5 paires de papilles postanales et 5 paires préanales, dont la plus antérieure est très loin en avant de l'extrémité proximale des spicules. P. Artigas (1930, p. 26, pl. IX, fig. 8) a vu 3 paires préanales et 3 postanales.

Dans les deux sexes, la partie antérieure des « baguettes œsophagiennes » est plus courte que la partie postérieure, comme cela a été décrit par Artigas pour multispinosa. 
Provenance : $a$ ) Intestin de Rhinocricus cachoeirensis O. Schubart; Etat de São-Paulo; 1900.

b) Intestin de Leptodesmus paulistus H. W. Brölemann, + ; de SãoSebastino (Etat de São-Paulo). D ${ }^{r}$ O. Schubart leg.

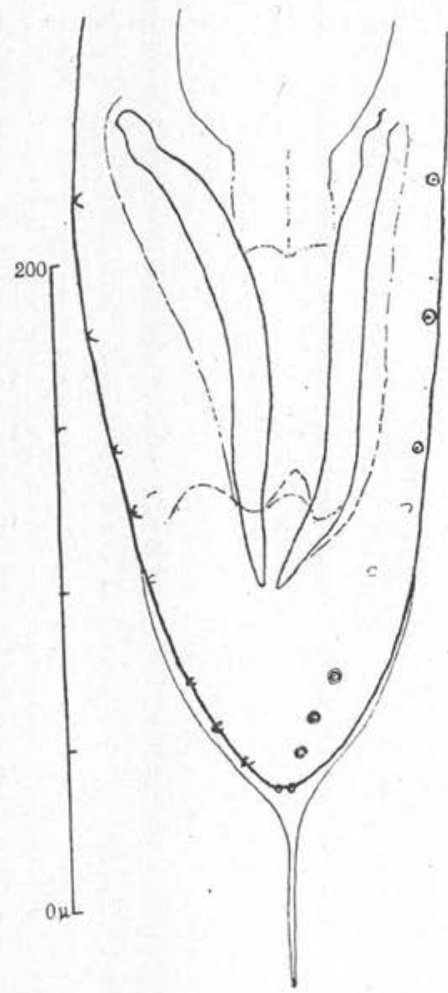

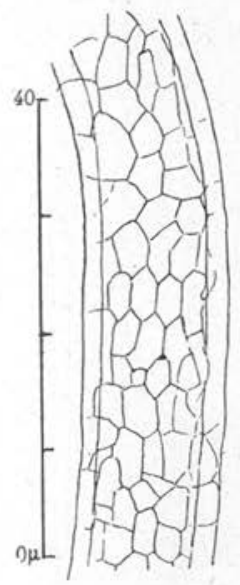

FIG. 26. - Même espèce et même provenance que pour les figures 23-25. Structure d'un spicule.

Fig. 25. - Même espèce et même provenance que pour les figures 23-24. Région postérieure d'un $\sigma^{*}$.

\section{RHIGONEMA GLABRUM n. sp.}

(Fig. 27-30)

La cuticule est très finement striée transversalement et dépourvue de pilosité. La queue est longue et effilée, beaucoup plus chez la + (fig. 30) que chez le $\delta$ (fig. 29). Les 6 «baguettes œsophagiennes » contenues dans le manchon musculaire œsophagien comportent une partie antérieure densément striée transversalement, et uné partie postérieure, séparée par une légère constriction, sans striation ou à striation indistincte. L'extrémité postérieure des baguettes œsophagiennes est légèrement courbée. La partie antérieure striée occupe environ deux tiers de la longueur de la baguette, la postérieure environ un tiers. 
La vulve est en arrière du milieu du corps, mais loin en avant de l'anus. Pour une $q$ mature longue de $4,724 \mathrm{~mm}$., large de 0,177 , la longueur prévulvaire est d'environ 2,408 et la longueur postvulvaire d'environ 2,316 , dont 0,720 pour la queue. Pour une $q$ immature, longue de $3,425 \mathrm{~mm}$, large de 0,13 , la queue est longue de 0,41 .

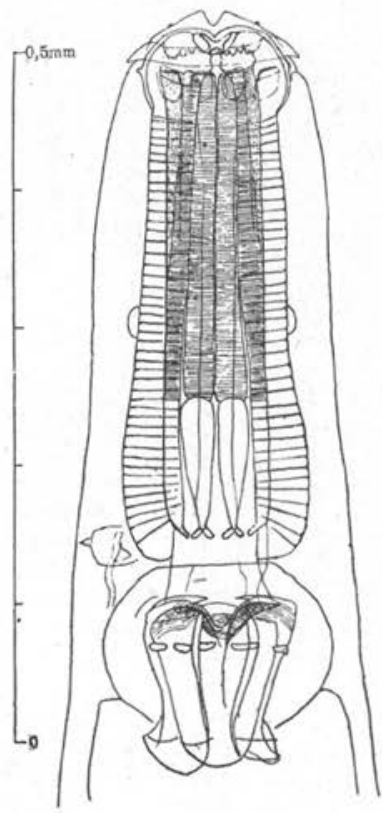

FiG. 27

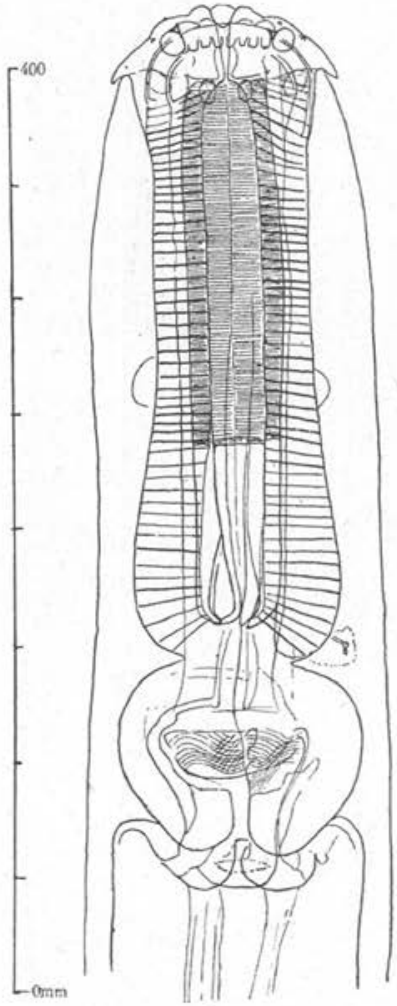

FiG. 28

Fig. 27. - Rhigonema glabrum n. sp. Intestin de Rhinocricus cachoeirensis O. Schubart. Etat de São Paulo (Brésil), 1900. Odette Tuzet ded. Région antérieure d'un $\delta$ long d'environ $6 \mathrm{~mm}$. 35, large d'environ $0 \mathrm{~mm}$. 46 .

FIG. 28. - Même espèce et même provenance que pour la figure 27. Région antérieure d'une of immature longue d'environ $3 \mathrm{~mm} .425$.

Les œufs sont peu nombreux (environ 9 à 32) dans l'utérus et mesurent environ $98 \times 86$ à $101 \times 89 \mu$, la coque est épaisse d'environ $5 \mu$.

Il n'y a pas de sac prévulvaire (ce qui correspond au genre Dudekemia P. Artigas). Les spicules, subégaux, sont très grêles et très longs (environ $1 / 7^{\circ}$ de la longueur totale du corps) ; pour un individu long d'environ $6,35 \mathrm{~mm}$., large d'environ 0,46 , ils atteignent environ 0,93 . 


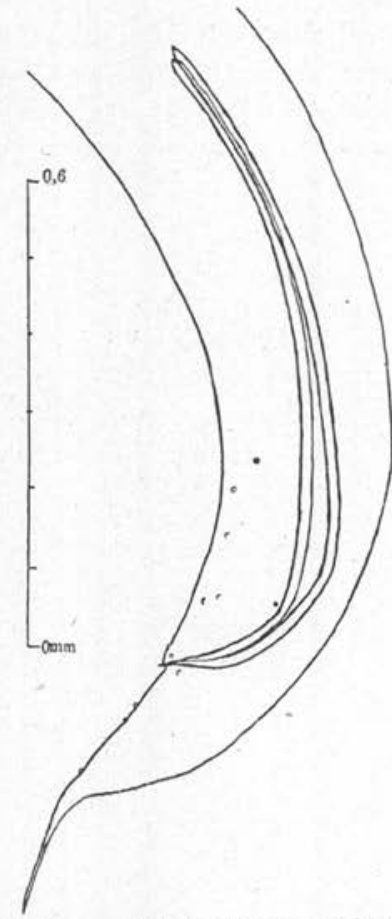

FIG. 29. - Mème espèce et mème provenance que pour les figures 27 et 28 . Région postérieure d'un individu long d'environ $6 \mathrm{~mm}$. 35, large d'environ $0 \mathrm{~mm}$. 46. Les spicules sont longs d'environ $0 \mathrm{~mm}$. 93 .

FIG. 30. - Mème espèce que pour les figures 27-29. Intestin de Rhinocricus sp., $\mathrm{n}^{\circ}$ 3.320. Environs idfEpisacio (Etat de Sảo Paulo, Brésil), 22-1-1950. Odette Tuzet ded. Queue d'une of longue d'environ $4 \mathrm{~mm}$. 724 (dont 0,720 pour la queue), large d'environ $0 \mathrm{~mm} .177$.

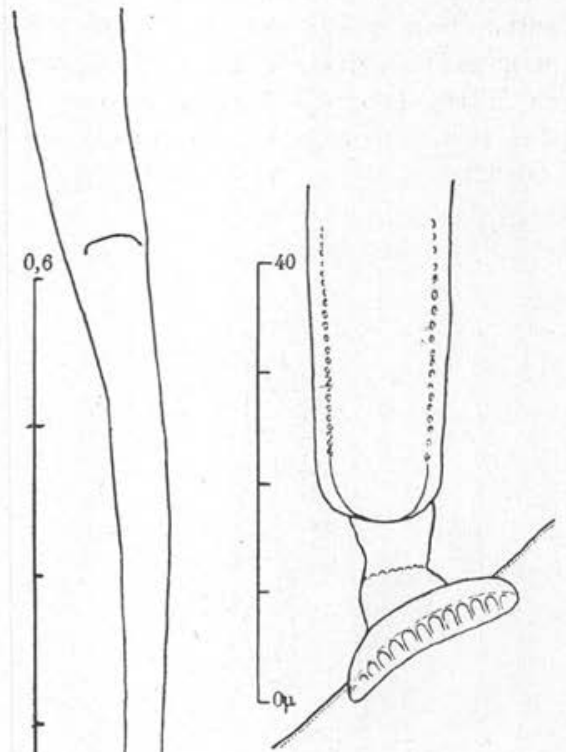

Fig. 31. - Région basale d'une Eccrinide indéterminée, fixée sur un Rhigonema glabrum $\mathrm{n}$. sp., à un niveau correspondant à la fiu du second tiers du corpus œsophagien. Intestin de Rhirocricus padbergi Verhoeff. Etat de São Paulo (Brésil), 1902. Odette Tuzet ded.

Provenance : a) Intestin de Rhinocricus cachoeirensis O. Schubart; Brésil, 1900 ; Etat de São-Paulo.

b) Intestin de Rhinocricus padbergi Verhoeff ; Brésil, 1902, Pirassununga (Etat de São-Paulo) (1).

(1) Presque tous les spécimens que j'ai examinés, provenant de $R h$. padbergi Verhoeff, portent de nombreux Eccrinides, dont on trouvera la description dans une publication de Mlles Odette Tuzet et Jehanne-Françoise Manier (Ann. Sc. Natur. Zool., 1952). 
c) Intestin de Rhinocricus sp.; environ d'Episacio (Etat de SãoPaulo), $\mathrm{n}^{\circ} 3320$; 22-1-1950.

d) Intestin de Leptodesmus jucundus W. H. Brölemann, . Etat de São-Paulo.

Discussion. - Cette espèce est à comparer avec les Rhigonema à tégument dépourvu de pilosité : brevicolle, acuminatum, multipapillatum, falcatum, subulatum, truncatum, simile. Chez toutes ces espèces, les spicules sont proportionnellement plus courts ; chez acuminatum, la longueur de la queue dépasse celle des spicules ; c'est le contraire chez glabrum ; il y a de longs spicules chez multipapillatum, leur longueur est double de celle de la queue, mais la longueur des spicules de glabrum est plus de deux fois celle de la queue ; chez subulatum, les spicules ont 1 fois $1 / 2$ la longueur de la queue ; chez glabrum, plus de 2 fois $1 / 2$. Il n'est donc pas possible de confondre glabrum avec les autres espèces à longs spicules et sans pilosité de Rhigonema.

\section{Genre Ichthyocephalus (P. Artigas 1926)}

Ce genre a été créé par P. Artigas (1926, p. $62 ; 1929$, p. 109) d'après une $\uparrow$; la diagnose a été complétée par J.-L. de Almeida (1933, p. 1193-1194), qui a eu à sa disposition plusieurs ơ et plusieurs ?.

\section{Ichthyocephalus ichthyocephaloides, n. sp.}

(Fig. 32-34)

De cette espèce, j’ai examiné seulement des $q$; elles diffèrent à peine de la description et des figures données pour le générotype par P. Artigas $(1926$, p. $62-63$, fig. 15 ; 1929 , p. 110-112, pl. XLIV, fig., pl. XLV, fig. 1-3), et les dimensions sont à peu près concordantes; cependant, il y a une différence très manifeste : sur les fines stries transversales de la cuticule, il y a des denticulations triangulaires. Aux faibles grossissements, ces denticulations semblent de forme très régulière et égales entre elles, mais aux forts grossissements (fig. 34) on constate leur irrégularité. Pour une $q$, longue de $6,1 \mathrm{~mm}$., large de 0,32 , la vulve est distante de $2,32 \mathrm{~mm}$. de l'extrémité caudale.

(1) Ce mot a été orthographié Icthyocephalus en 1926 par ArTigas et Ichtyocephalus en 1933 par .J.-L. de ALmeIDA; la transcription correcte est Ichthyocephalus; c'est celle adoptée par Paul-A. RÉmy (The Naturalist, London, julysept. 1950 , p. 104). 


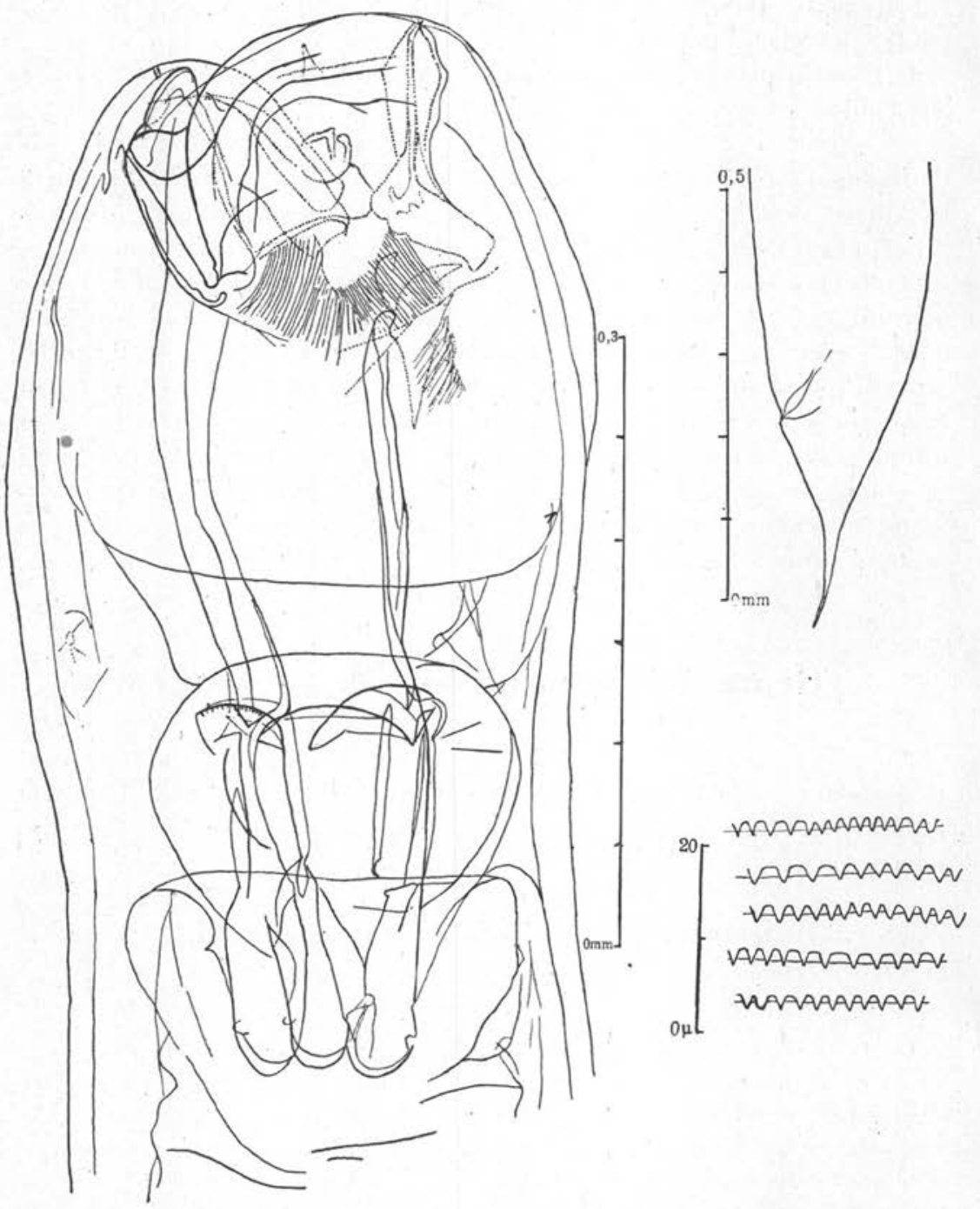

Fig. 32. - Ichthyocephalus ichthyocephaloides n. sp. Intestin de Rhinocricus sp., $\mathrm{n}^{\circ}$ 3.320. Environs d'Episacio (Etat de São Paulo, Brésil), 22-1-1950. Odette Tuzet ded. Région antérieure d'une $q$ à maturité longue d'environ $6 \mathrm{~mm}$. 1, large de $0 \mathrm{~mm}$. 32 .

Fig. 33. - Même espèce et mème provenance que pour la figire 32. Extrémité postérieure d'une $q$ longue d'environ $5 \mathrm{~mm}$. 42, large d'environ $0 \mathrm{~mm}$. 23 à $0 \mathrm{~mm} .27$.

Fig. 34. - Même espèce et même provenance que pour les figures 32 et 33 . Ornementation cuticulaire d'une $q$ à maturité longue d'environ $6 \mathrm{~mm} .1$. 
Les œufs utérins, ellipsoïdaux, mesurent, en général, de $147 \times 112 \mu$, à $161 \times 124 \mu$ (j'ai mesuré aussi $150 \times 118,156 \times 127,159 \times 118$, $161 \times 132)$; la coque a une épaisseur d'environ $5,5 \mu$.

Provenance : Intestin de Rhinocricus sp.; environs d'Episacio (Etat de São-Paulo, Brésil) ; n $3320 ; 22-1-1950$.

\section{Ichthyocephalus Almeidai n. sp.}

(Fig. 35-39)

Cette espèce diffère manifestement du générotype et d'ichthyocephaloides par la plus grande longueur du corpus œsophagien reliant le « bulbe buccal » au bulbe œesophagien (= gésier).

Les spicules, minces et grêles, ont une longueur supérieure au dixième de la longueur totale de l'individu $(430 \mu$, pour un individu long d'envi-

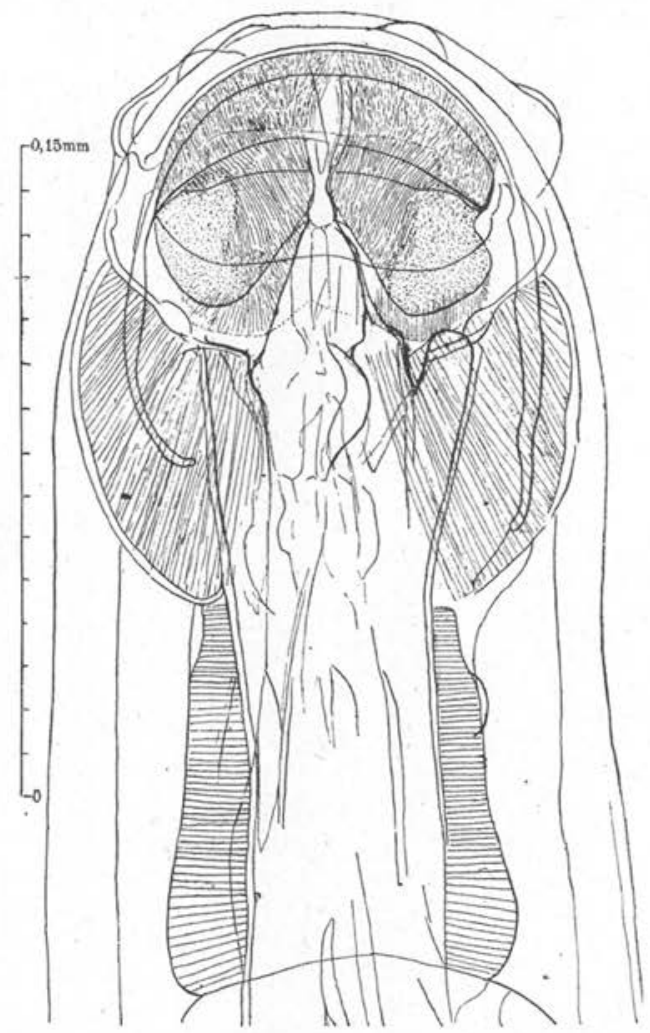

FIG. 35. - Ichthyocephalus Almeidai n. sp. Intestin d'Hemigymnostreptus sp. Santa Adelia (Etat de São Paulo, Brésil), $\mathrm{n}^{\circ} 3.281,7-1-1950$. Odette Tuzet ded. Région antérieure d'un $\delta$ long d'environ $3 \mathrm{~mm}$. 38, large d'environ $0 \mathrm{~mm} .208$. 


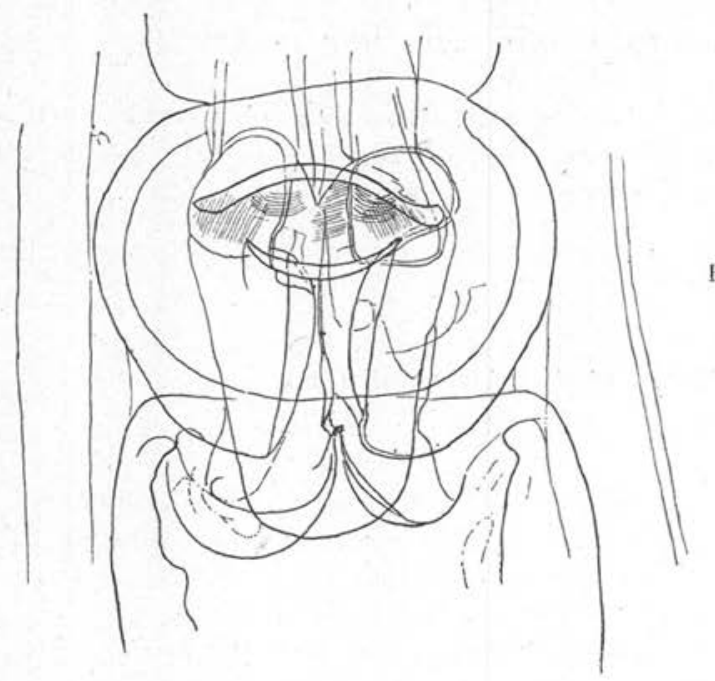

FIG. 36. - Même spécimen que celui de la figure 35. Même grossissement. A p pareil masticateur.

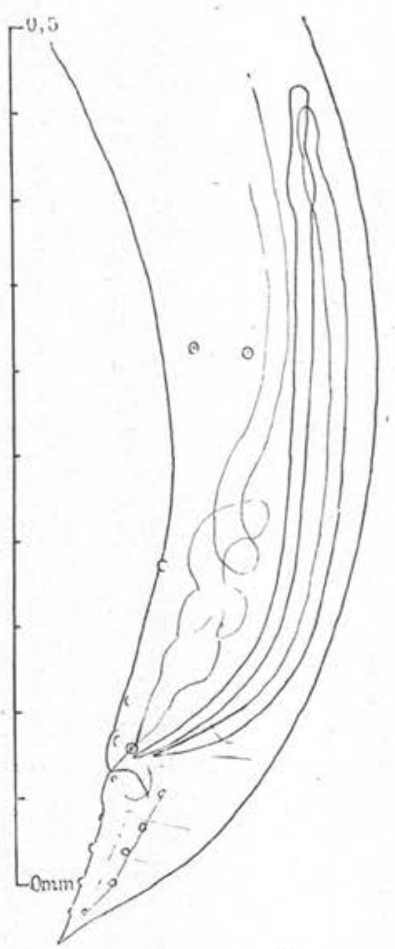

FIG. 37

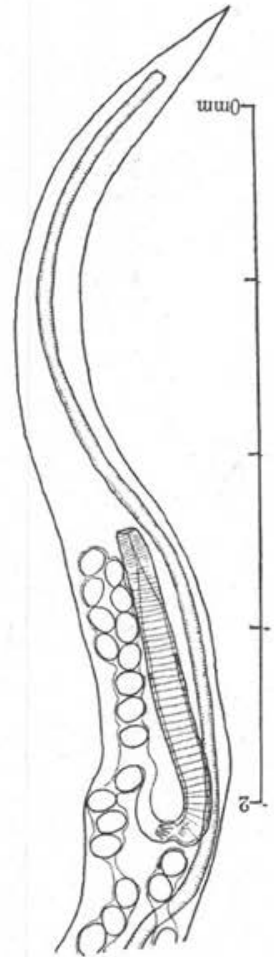

Fig. 39

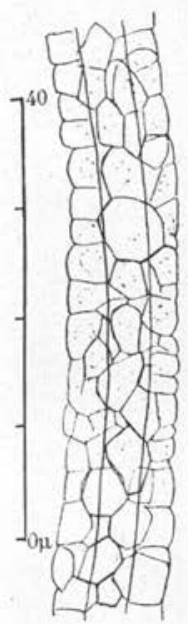

F1G. 38

Fig. 37. - Même espèce et mème provenance que pour les figures 35-36. Région postérieure d'un ¿ long d'environ $3 \mathrm{~mm}$. 15, large d'environ $0 \mathrm{~mm}$. 19 .

Fig. 38. - Même espèce et même provenance que pour les figures 35-37. Structure des spicules.

FIG. 39. - Mème espèce et même provenance que pour les figures 35-38. Région postérieure d'une $q$ longue d'environ $4 \mathrm{~mm}$. 15 (dont 0,26 pour la queue), large d'environ $0 \mathrm{~mm}$. 31. La vulve est à environ $2 \mathrm{~mm}$. 50 de l'extrémité antérieure et $1 \mathrm{~mm} .65$ de l'extrémité postérieure. 
ron $3,15 \mathrm{~mm}$.) ; ils sont très caractéristiques, en raison de leur structure micropolyédrique (fig. 38). Chez ichthyocephaloides, les spicules ne présentent pas cette structure et sont plus gros.

L'ovéjecteur (vagina vera) (fig. 39) est proportionnellement beaucoup plus long que chez artigasi. Les œufs utérins mesurent environ $85 \times 70 \mu$ (j'ai mesuré aussi $74 \times 63,81 \times 66,86 \times 66,86 \times 72,86 \times 74 \mu$ ), chez des 9 , parasites d'un Hemigymnostreptus; ils atteignent jusqu'à $98 \times 72 \mu$ (j'ai mesuré $86 \times 74,89 \times 69,89 \times 72,89 \times 74,91 \times 69,94 \times 78$, $97 \times 72,98 \times 72$ ), chez des $q$ parasites d'un Gymnostreptus.

Provenance : $1^{\circ}$ Intestin d'Hemigymnostreptus sp.; Santa Adelia (Etat de São-Paulo, Brésil), $\mathrm{n}^{\circ}$ 3281; 7-1-1951.

$2^{\circ}$ Intestin de Gymnostreptus sp.; Santa Adelia (Etat de São-Paulo, Brésil) ; $\mathrm{n}^{\circ} 3283 ; 7-1-1950$.
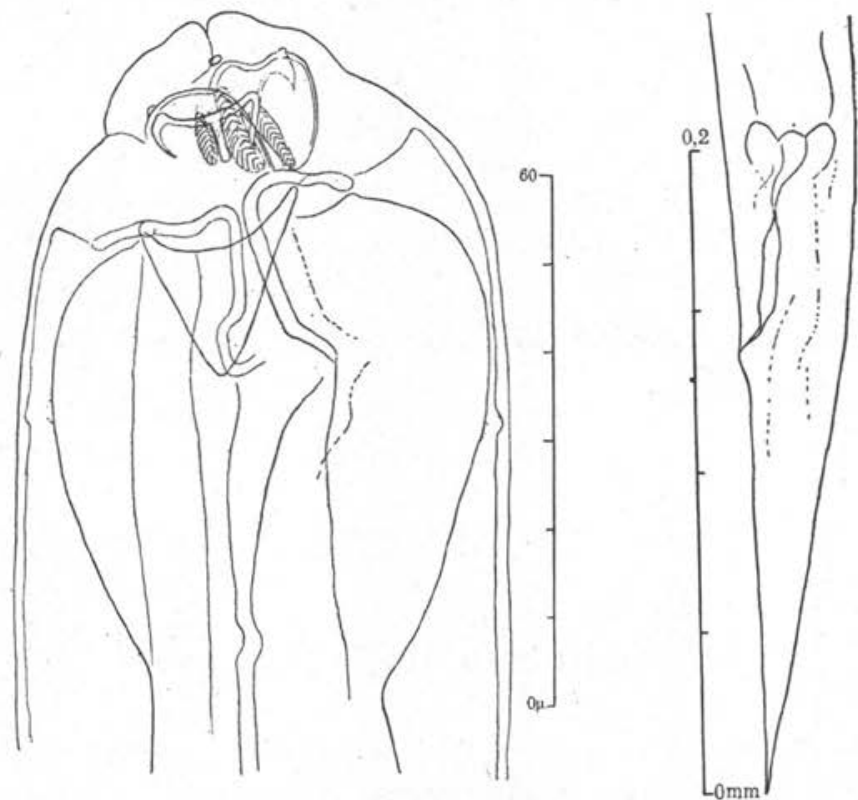

Fig. 40. - Ichthyocephalus sp. Intestin d'Hemigymnostreptus sp. ; Santa Adelfa (Etat de São Paulo, Brésil), $\mathrm{n}^{\circ}$ 3.281. Odette Tuzet ded. Région antérieure d'une $\subsetneq$ immature longue d'environ $1 \mathrm{~mm}$. 38 (dont 0,140 pour la queue), large d'environ $0 \mathrm{~mm}$. 06 .

Fig. 41. - Même individu que celui de la fig. 40 ; forme de la queue.

\section{Ichthyocephalus sp.}

(Fig. 40-41)

Une petite $q$ immature ne me semble pas identifiable à l'une des espèces précédentes. Dans la cavité buccale se trouvent trois formations 
oblongues, portant des stries disposées en chevrons. Cet immature est long de $1,38 \mathrm{~mm}$., dont 0,140 pour la queue ; sa largeur est de 0,06 . La tête, en avant de la partie antérieure dilatée de l'œsophage, a une longueur de 0,023 ; la partie antérieure dilatée de l'œsophage mesure $0,049 \times 0,023$; le corpus œsophagien cylindrique mesure $0,054 \times 0,028$; le bulbe $0,043 \times 0,043$. L'appareil masticateur du bulbe se prolonge dans le début de l'intestin comme chez toutes les espèces du genre. La partie postérieure du corps s'atténue en pointe, sans brusque rétrécissement. L'emplacement de la vulve n'est pas observable.

Provenance : Intestin d' Hemigymnostreptus sp., Santa Adelia (Etat de São-Paulo, Brésil), $\mathrm{n}^{\circ}$ 3281, 7-1-1951.

\section{Famille ATRACTIDE (L. Travassos 1920)}

Sur les quatre sous-familles actuellement placées dans cette famille : Atractinx A. Railliet 1917, Labiduridx W. Yorke et P. A. Maplestone 1926, Ransomnematinæ (L. Travassos 1929), Cruznematinæ (P. Artigas 1929), les deux dernières sont représentées chez des Myriapodes et il semble qu'elles leur sont particulières.

\section{Sous amille RANSOMNEMATINAE (L. Travassos 1929)}

Les genres de cette sous-famille se répartissent en deux groupes : l'un où le $\delta$ est pourvu d'une ventouse préanale (genres Ransomnema, Clementeia, Heth-Streptogaster), l'autre où le ơ est dépourvu de ventouse (genres Carnoya, Rondonema, Angra) (1).

\section{Ransomnema (P. Artigas 1926)}

Voir la diagnose du genre in P. Artigas, 1926, p. 38 ; 1929, p. 65. Ce genre a été créé pour deux espèces : $R$. ransomi P. Artigas 1926 (générotype) et $R$. longispiculum P. Artigas 1926; une troisième espèce, bien typique du genre, est Ransomnema macrocephalus (J. d'Udekem 1856), excellemment décrite et figurée par J. d'Udekem, comme le rappelle P. Artigas (1930, p. 22).

(1) Dans la classification de I. N. FinipJEv et J. H. S. Stekhoven (1941, p. 837), les trois genres Ransomnema, Heth, Clementeia sont seuls admis dans la sousfamille Ransomnematina qui est placée dans la fam. Kathlaniida, et les autres genres sont répartis différemment : Rondonema et Carnoya dans la sous-famille Carnoyaina, placée dans la fam. Cosmocercida; Angra dans la sous-fam. Syphacina de la fam. Oxyurida. La sous-famille Cruznematina n'est pas admise et Cruznema est dans la sous-famille Kathlaniinz. 
Chez les deux espèces de Ransomnema que j'ai examinées, l'isthme œsophagien est délimité du bulbe, ce n'est pas un « goulot du bulbe »; il ne fait pas partie du bulbe, alors que, dans les genres Heth, Rondonema, Clementeia, l'isthme est un prolongement du bulbe, sans démarcation.

Les $\widehat{\sigma}$ de Ransomnema ont toujours 2 spicules inégaux, sans gubernaculum, et une ventouse ventrale préanale.

\section{RANSOMNEMA CHRISTIEI n. sp.}

(Fig. 42-45)

La cavité buccale a une organisation compliquée, mal distincte chez mes spécimens.

Dans le manchon musculaire sesophagien, se trouvent trois lames longitudinales, striées transversalement et qui forment chacune un angle dièdre - comme cela a été décrit par J. d'Udekem (1856, p. 561, pl. II, fig. 4-5) pour Ransomnema macrocephalus (J. d'Udekem) - elles correspondent à six baguettes œsophagiennes.

․ La $q$ a une queue proportionnellement plus courte que chez macrocephalus, et la vulve est située plus loin, antérieurement, de l'anus.

Une $q$ mature, longue de $1,724 \mathrm{~mm}$, large de $0,150-0,152$, a sa vulve distante de 0,58 de l'extrémité postérieure ; une autre $q$ mature, longue de 1,70 , large de 0,165 , a sa vulve distante de 0,55 de l'extrémité postérieure. La queue est plus courte que chez ransomi (cf. Artigas, 1926, p. 43, fig. 7). Les œufs utérins mesurent $146-150 \times 85-90 \mu$, ils sont plus gros que ceux de ransomi $(130 \times 55 \mathrm{j})$.

$\sigma^{2}$. Le setil $\delta$ complet à ma disposition est long de $1,52 \mathrm{~mm}$., dont 0,125 pour la queue, large de 0,085 . Le corpus œsophagien est long de 0,23 , large de 0,034 , l'isthme est long de 0,066 , le bulbe a un diamètre de 0,059 . La ventouse mesure longitudinalement $32 \mu$, son centre est à 0,182 en avant de l'anus, et à environ 1,40 de l'extrémité antérieure. Le grand spicule est long de 0,22 , le petit long de 0,09 . On remarquera que l'extrémité proximale du grand spicule atteint le niveau du bord postérieur de la ventouse, caractère qui permet immédiatement de distinguer cette espèce de ransomi chez qui elle ne l'atteint pas.

Provenance : Intestin de Rhinocricus cachoeirensis O. Schubart. Etat de São-Paulo, 1900. 


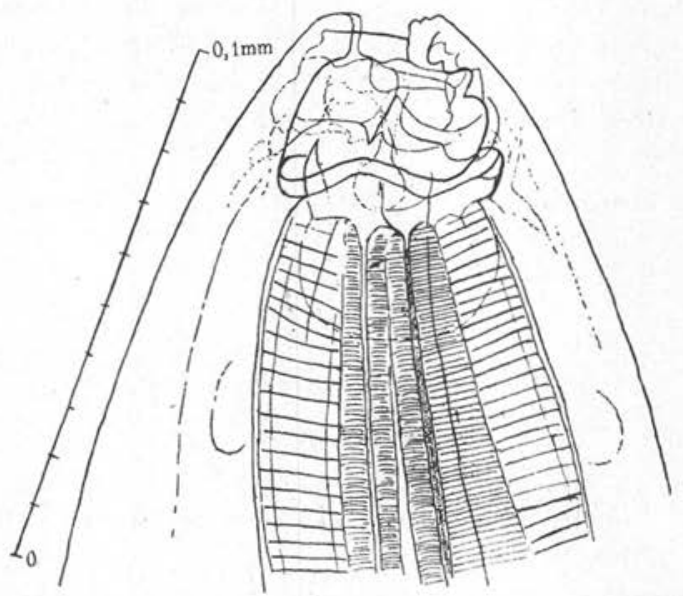

FiG. 42. - Ransomnema Christiei n.sp. Intestin de Rhinocricus cachoeirensis O. Schubart. Etat de São Paulo, Brésil, 1900. Odette Tuzet ded. Extrémité antérieure d'une $q$ à maturité.

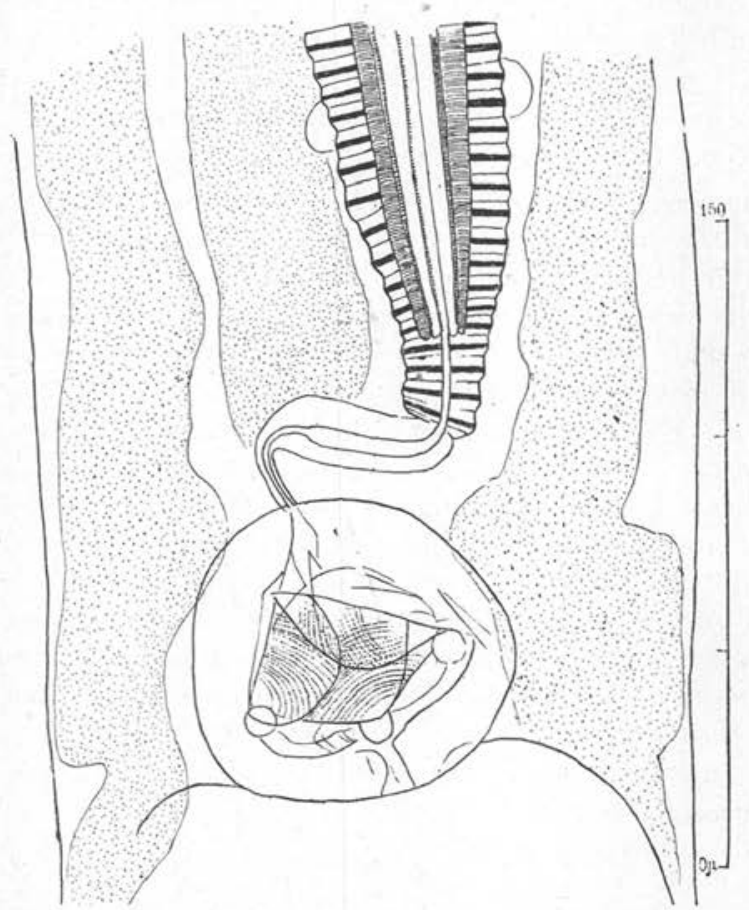

Fıg. 43. - Mème espèce et même provenance que pour la figure 42. Région de l'isthme œsophagien et du bulbe, chez une + à maturité, longue d'environ $1 \mathrm{~mm} .70$ (dont $1 \mathrm{~mm}$. 16 avant la vulve), large d'environ $0 \mathrm{~mm} .165$. 

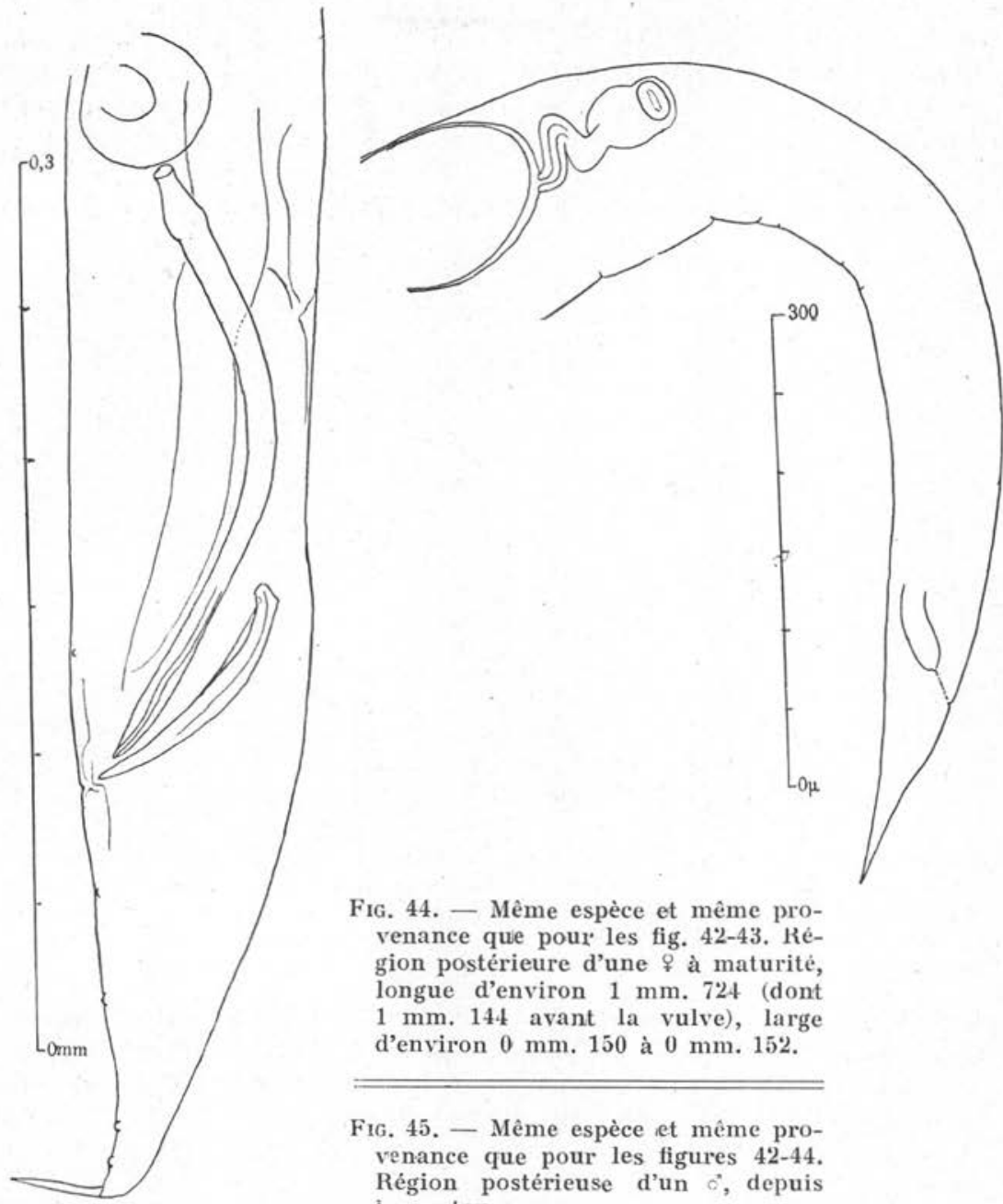

Fig. 44. - Même espèce et même provenance que pour les fig. $42-43$. Région postérieure d'une $\$$ à maturité, longue d'environ $1 \mathrm{~mm}$. 724 (dont $1 \mathrm{~mm} .144$ avant la vulve), large d'environ $0 \mathrm{~mm}$. 150 à $0 \mathrm{~mm}$. 152 .

Fig. 45. - Même espèce et même provenance que pour les figures 42-44. Région postérieuse d'un $\circ^{\circ}$, depuis la ventouse.

RANSOMNEMA LONGISPICULUM (P. Artigas 1926)

(Fig. 46-48)

Cette espèce ayant déjà été décrite par P. Artigas (1926 et 1929), je me limiterai à quelques remarques.

P. Artigas ne dit rien de la capsule buccale; celle-ci est large et profonde et contient trois brèves projections cuticulaires, une dorsale et deux subventrales, qui sont quelque peu pennées, rappelant celles décri- 
tes par M. B. Chitwood (1935, p. 50, fig. 8 B) chez le ơ de Heth dimorphum M. B. Chitwood; elles sont plus développées que celles « shaped somewhat like palm leaves » observées par le binôme Chitwood, chez Thelastoma palmeltum B. G. Chitwood et M. B. Chitwood (1934, pl. I, fig. 4).

․ Pour un individu long de $1,788 \mathrm{~mm}$., dont 0,138 pour la queue, large

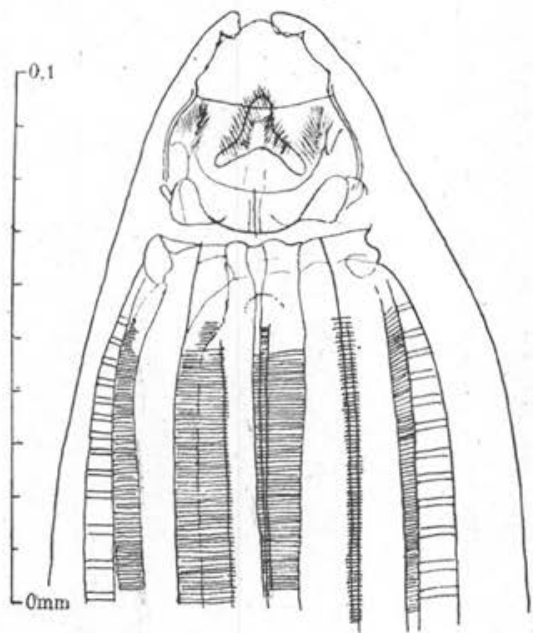

FIG. 46. - Ransomnema longispiculum P. Artigas. Intestin de Rhinocricus sp. Environ d'Episacio (Etat de São Paulo, Brésil), $\mathrm{n}^{\circ} 3.320,22-1-1950$. Odette Tuzet ded. Région antérieure d'une $\subsetneq$ longue d'environ $1 \mathrm{~mm} .52$ (jusqu'à la vulve $0 \mathrm{~mm}$. 97 ; jusqu'à l'anus $1 \mathrm{~mm}$. 42), large d'environ $0 \mathrm{~mm} .13$.

de 0,167 , la vulve est distante de l'anus de 0,40 . Pour un autre, long de $1,52 \mathrm{~mm}$., dont 0,10 pour la queue, large de 0,13 , la vulve est distante de l'anus de 0,55. La vulve est donc proportionnellement plus près de l'anus que chez l'espèce précédente et chez ransomi.

Les œufs utérins mesurent de $130 \times 105 \mu$ à $157 \times 95 \mu$.

Provenance : a) Intestin de Rhinocricus padbergi O. Schubart. Etat de São-Paulo, 1902.

b) Intestin de Rhinocricus sp. Environs d'Episacio (Etat de SãoPaulo), $\mathrm{n}^{\circ} 3320 ; 22-1-1950$.

\section{Rondonema P. Artigas 1926}

Ce genre, très voisin de Carnoya, a été créé pour une seule espèce (rondoni), dont le $\delta$ et la $q$ ont été trouvés par P. Artigas dans l'intestin d'un Diplopode de l'Etat de São-Paulo. Chez cette espèce, la ণ, sur la partie antérieure du corps, dans la région prébulbaire, porte, 

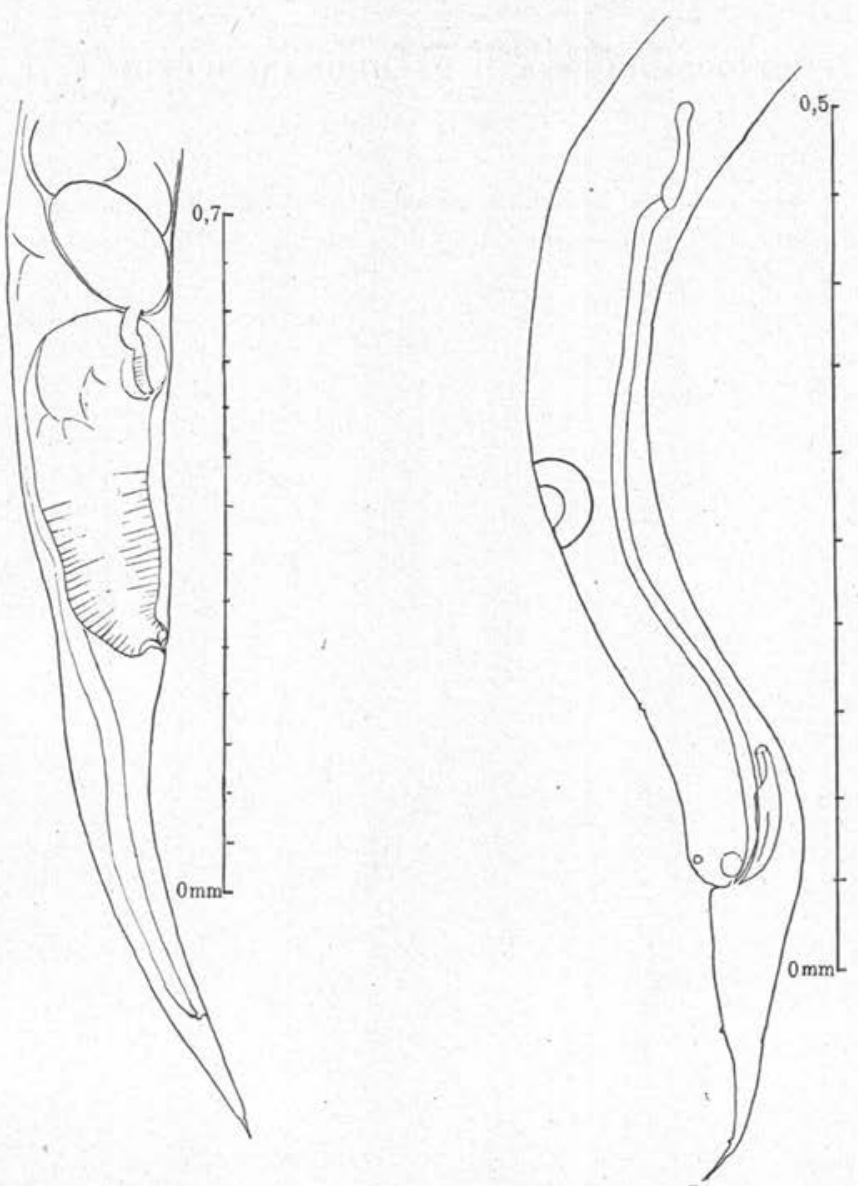

FIG. 47. - Même espèce que pour la figure 46. Intestin de Rhinocricus padbergi Verhoeff. Etat de São Paulo (Brésil), 1902. Odette Tuzet ded. Région postérieure d'un $\sigma^{*}$ long d'environ $1 \mathrm{~mm} .72$ (dont $0 \mathrm{~mm}$. 175 pour la queue), large d'environ $0 \mathrm{~mm}$. 078. Le grand spicule est long d'environ $0 \mathrm{~mm}$. 470, le petit d'environ $0 \mathrm{~mm}$. $095-0 \mathrm{~mm}$. 100. La ventouse mesure $34 \times 17 \mu$ et son centre est à $240 \mu$ de l'anus.

FıG. 48. - Même espèce et même provenance que pour la fig. 47. Région postérieure d'une $f$ longue d'environ $1 \mathrm{~mm}$. 788, dont $0 \mathrm{~mm}$. 138 pour la queue ; entre la vulve et l'anus $0 \mathrm{~mm}$. 4 .

à droite et à gauche, une suite de six fortes épines égales entre elles, disposées longitudinalement le long des champs latéraux. Le ơ n'a pas d'épines ; il est pourvu, dit Artigas (1926, p. 59-60 ; 1929 , p. 72, 81), de deux spicules reposant sur un gubernaculum. Chez l'espèce que je rapporte ci-dessous, avec beaucoup d'hésitation, à Rondonema, la $q$ ne porte pas d'épines.

Ann. de Parasitologie, T. XXVII, N ${ }^{\circ} 1-2-3 .-1952$. 


\section{RONDONEMA (gen. ?) PSEUDONANNOLENOU n. sp.}

(Fig. 49-56)

․ La cuticule, très finement striée transversalement, ne porte ni épines ni poils. Les lèvres buccales ne sont pas saillantes, il semble qu'il

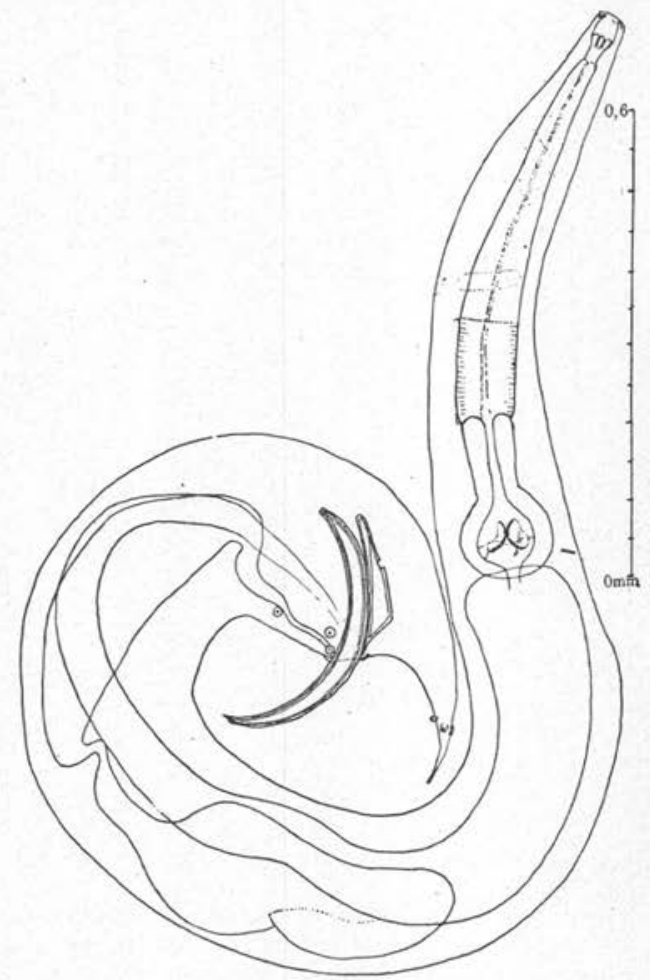

Fig. 49. - Rondonema pseudonannolenou n. sp. Intestin de Pseudonannolene tricolor H. W. Brölemann. Pirassununga (Etat de São Paulo, Brésil), 1888. Odette Tuzet ded. Spécimen ơ ; l'extrémité du prolongement caudal est brisée.

y ait 4 papilles. La cavité buccale est peu profonde, elle est suivie par un præcorpus œsophagien, cylindrique, à lumière étroite, qui se continue par le postcorpus œsophagien, beaucoup plus gros, et beaucoup plus long, botuliforme, dont la cavité contient 6 baguettes œsophagiennes, celles-ci montrent une démarcation transversale vers la fin de leur troisième cinquième, et se terminent par une pièce très courte, en forme de crochet. L'isthme œsophagien est d'un diamètre plus faible que celui du corpus auquel il fait suite; c'est le « goulot » du bulbe, dont il est 
le prolongement sans démarcation. Le bulbe contient un puissant appareil masticateur.

L'ovéjecteur est très long, avec une forte musculature ; la vulve est en arrière de la mi-longueur du corps ; les œufs utérins sont peu nombreux (en général 8 à 10) et très gros, mesurant environ 140-146 $\times 85$-95 $\mu$, $152 \times 90 \mu, 152 \times 101 \mu$, et jusqu'à $159 \times 98 \mu$. La queue s'atténue en

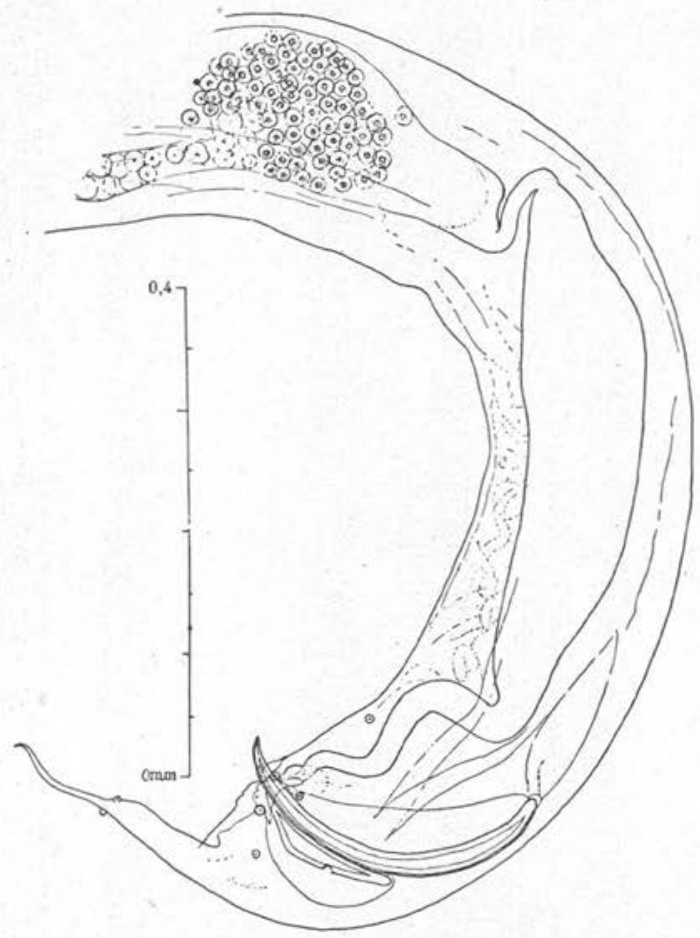

FIG. 50. - Même espèce et même provenance que pour la figure 49. Région postérieure d'un $\sigma^{\prime}$.

pointe, elle est beaucoup plus courte que chez $R$. rondoni P. Artigas.

Pour une $q$ longue de $2,807 \mathrm{~mm}$., dont 0,295 pour la queue, large de 0,290 au niveau de la vulve, le præcorpus œsophagien est long de 0,085 , large de 0,017 , le corpus long de 0,220 , large de 0,070 , le bulbe a un diamètre de 0,135 . L'isthme est long de 0,085 , large de 0,025 . La vulve est à 1,332 de l'extrémité antérieure et à 1,180 en avant de l'anus.

๙. L'extrémité antérieure est subtronquée, large, avec 2 lèvres peu saillantes et 4 papilles; la cavité buccale est profonde et comprend une partie antérieure, large, et une partie postérieure étroite ; entre les deux parties, il y a un épaississement cuticulaire dessinant 3 lobes. L'œsophage n'a pas de præcorpus ; le corpus est subcylindrique, long et grêle, 
augmentant un peu de diamètre dans sa moitié postérieure; il semble que, dans son dernier quart, sa structure soit un peu différente. Il est suivi, avec démarcation, par l'isthme qui est un prolongement du bulbe
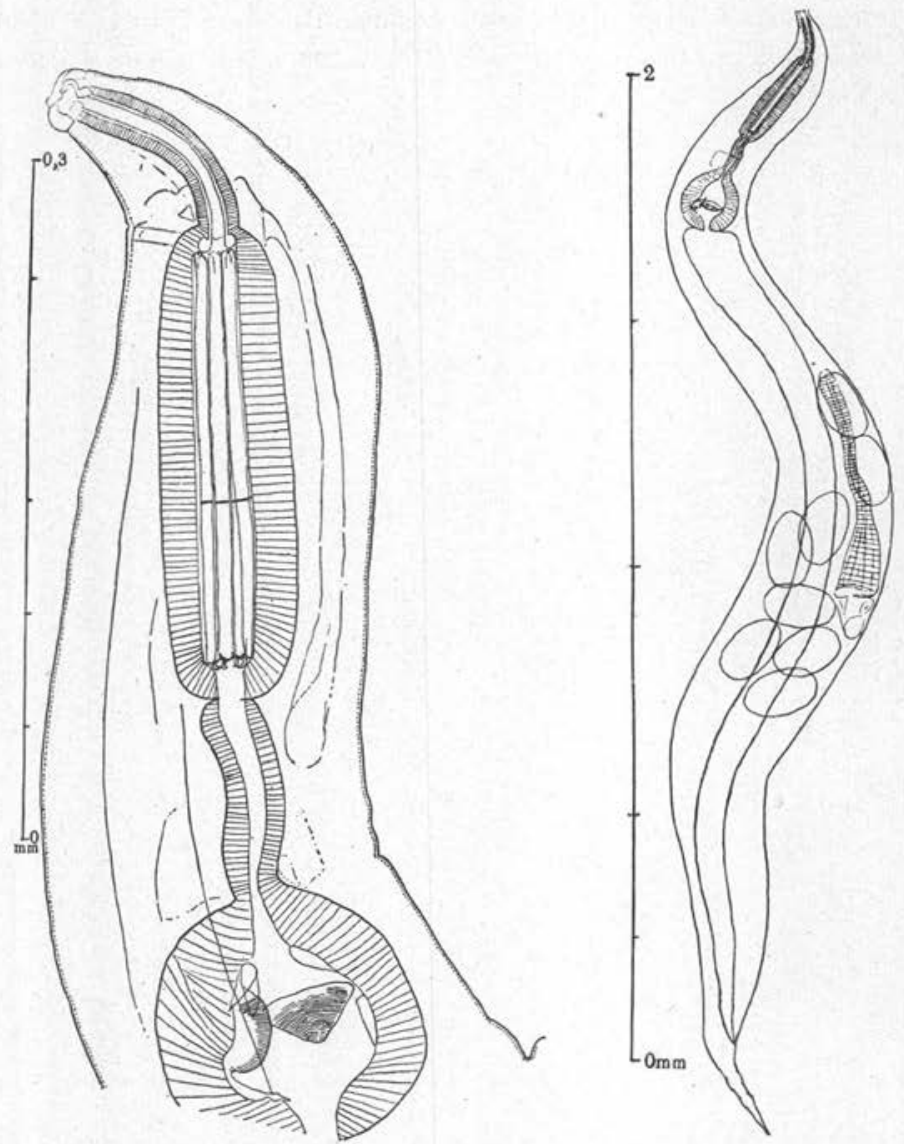

Fig. 51. - Même espèce et même provenance que pour les figures 49-50. Région antérieure d'une ${ }^{\circ}$.

Fig. 52. - Même espèce et même provenance que pour les figures 49-51. Femelle à maturité.

(goulot du bulbe) et aussi large, ou presque, que le corpus œesophagien. Le bulbe contient un puissant appareil masticateur.

La vésicule séminale et le vas deferens sont très développés ; ce dernier se rétrécit pour former, sans démarcation, le canal éjaculateur. Il y a deux spicules égaux arqués et un gubernaculum. En vue latérale, il est difficile de voir qu'il y a deux spicules, ceux-ci étant appliqués l'un 


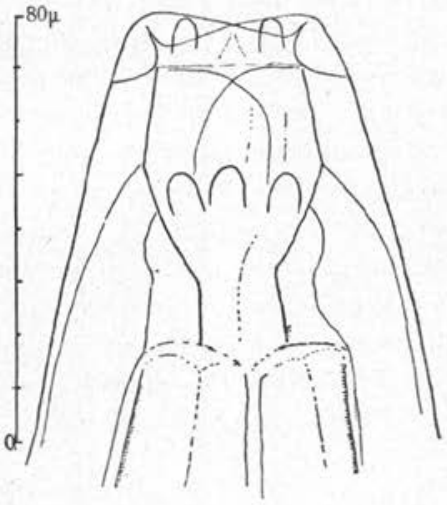

FiG. 53

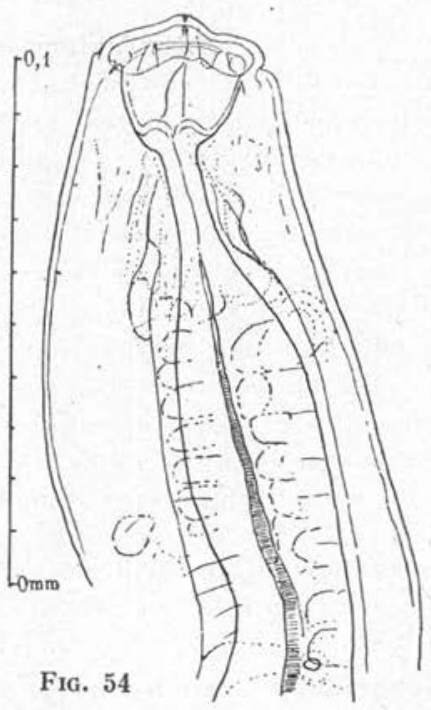

Fig. 53. - Même espèce et même provenance que pour les figures 49-52. Extrémité antérieure d'un ơ.

FIG. 54. - Même espèce et même provenance que pour les figures 49-53. Région antérieure d'un $\sigma^{\circ}$.
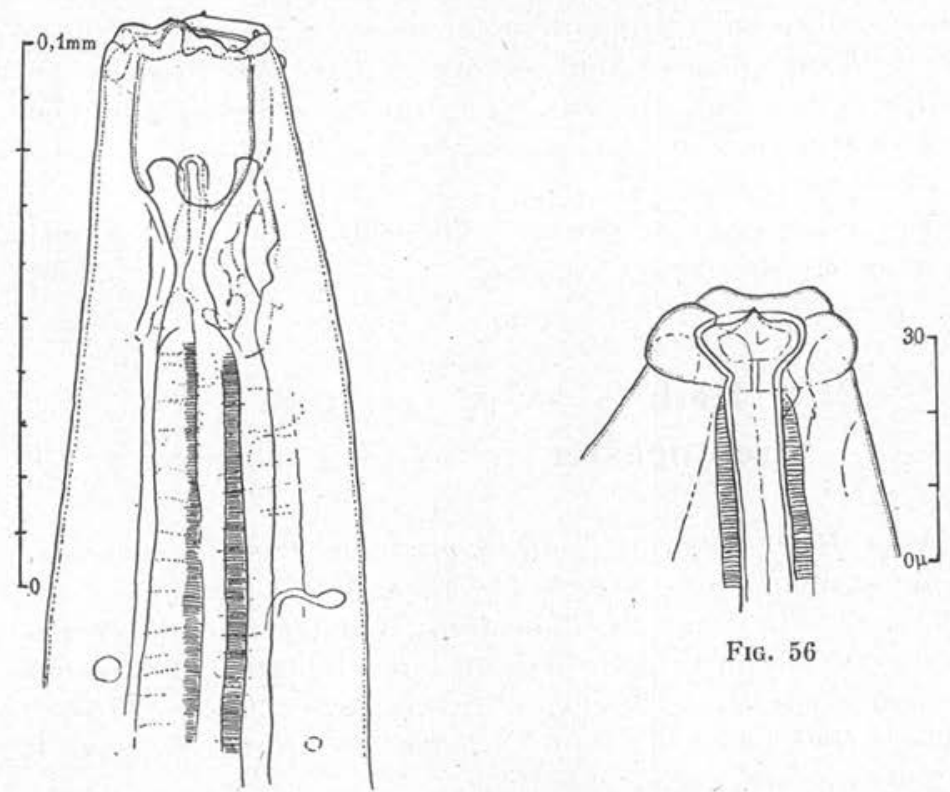

FIG. 56

Fig. 55. - Même espèce et même provenance que pour les figures 49-54. Région antérieure d'un $\sigma^{\circ}$.

Fig. 56. - Même espèce et même provenance que pour les figures 49-55. Région antérieure d'unc f. 
contre l'autre. Le gubernaculum est triangulaire et naviforme ; son bord dorsal présente une échancrure constante et très nette chez les quatre individus que j'ai examinés. La structure des spicules et du gubernaculum paraìt subréticulée ou plutòt spongieuse. Un peu après l'anus, la queue se rétrécit assez brusquement et s'atténue en pointe presque filiforme; cette partie atténuée porte ventralement une paire de papilles et dorsalement une papille. Plus antérieurement, il y a deux paires de papilles postanales et trois paires préanales.

Un mâle est long de $2,34 \mathrm{~mm}$., dont 0,224 pour la queue, avec une plus grande largeur de 0,187 ; son corpus œsophagien est long de 0,382 , avec une plus grande largeur de 0,040 ; l'isthme est long de 0,075 , avec une largeur de 0,030 , le bulbe a un diamètre de 0,105 . Les spicules sont longs de 0,190 , le gubernaculum long de 0,125 .

Provenance : Intestin de Pseudonannolene tricolor H. W. Brölemann, Etat de São-Paulo, 1886 et 1888.

Discussion. - Ainsi que je l'ai dit plus haut, je ne rapporte qu'avec doute cette espèce au genre Rondonema, la $q$ étant dépourvue, dans la région préintestinale, des deux files de six épines existant chez le générotype ; mais il y a, chez la $q$ comme chez le ô, des ressemblances avec le générotype qui me semblent assez importantes pour justifier une attribution au même genre. La forme et la structure du tube digestif sont les mêmes, l'ovéjecteur est aussi très développé et long, les spicules, ainsi que le gubernaculum ont la même forme et la même disposition, les papilles caudales sont semblablement disposées.

Il serait intéressant de savoir si l'hôte du générotype appartient aussi au sous-ordre Nannolenoidea.

\section{Heth [] (N. A. Cobb 1898) \\ $=$ Streptogaster $\left[\sigma^{\star}\right]$ (N. A. Cobb 1898)}

Le genre Heth apparaît pour la première fois dans la légende d'une figure donnée par N. A. Cobb (1898, p. 299, fig. 10), pour la $q$ d'un Nématode de l'intestin d'un Julus indéterminé de NouvelleGalles du Sud. D’après cette légende, une tentative de diagnose a été présentée par H. A. Baylis et R. Daubney (1926, p. 45-46), qui ont placé Heth dans la fam. Rhabditidæ H. Micoletzky 1922, s.-f. Rhabditinæ H. Micoletzky 1922.

Le genre Streptogaster apparaît pour la première fois dans la légende d'une figure donnée par N. A. Cobb (1898, p. 320, fig. 46) 
pour le $\delta$ d'un Nématode dont la provenance n'est pas indiquée. D'après cette légende, une tentative de diagnose a été présentée par H. A. Baylis et R. Daubney (1926, p. 60), qui ont placé Streptogaster en appendice aux Rhabditidx. Auparavant, Heth et Streptogaster avaient été considérés par L. Travassos (1919, p. 61) comme appartenant à la sous-famille Hystrignathinæ L. Travassos 1919, famille Lepidonematidæ (L. Travassos 1919).

P. Artigas (in L. Travassos, 1929, p. 20) reconnut que Streptogaster était le ơ de Heth, et L. Travassos (1929, p. 24) attribua alors le genre à la famille Thelastomatidæ (L. Travassos 1929), sous-famille Ransomnematinæ (L. Travassos 1929).

P. Artigas (1929, p. 19) accepta cette attribution aux Thelastomatinæ, mais plaça les Thelastomatidæ dans les Oxyuroidea, alors que Travassos en avait fait des Rhabdiasoidea, malgré A. Railliet et A. Henry (1916, p. 115) qui avaient reconnu, les premiers, que Heth appartenait aux Oxyurides.

Actuellement, les Ransomnematinæ sont exclus des Thelastomatidæ, et l'on s'accorde avec B. G. et M. B. Chitwood (1937, p. 50 ; 1950, p. 18) pour les admettre dans les Atractidæ L. Travassos 1920.

Pour une diagnose générique de Heth-Streptogaster, l'on a recours à celles données par H. A. Baylis et R. Daubney (1926, p. 4546,60 ) et par P. Artigas (1929, p. 81-82), mais aucune n'est bien satisfaisante : par exemple, Baylis et Daubney, interprétant Cobb, disent que, apparemment, il y a un seul spicule et deux pièces accessoires ; P. Artigas, d'après ce qu'il a observé, dit « un seul spicule avec gubernaculum », alors que M. B. Chitwood (1935, p. 5051) figure deux spicules et les décrit comme « presque complètement fusionnés ».

Chez les spécimens que je considère comme des ô de Heth, il y a deux spicules très inégaux, non fusionnés, dont le plus petit pourrait, peut-être, être interprété comme pièce accessoire (gubernaculum).

Ont été décrites dans le genre Heth ou attribuées à ce genre :

a) Trois espèces dont on connaît le $\delta$ et la $q$ :

Heth juli N. A. Cobb $1898\left(\sigma^{\circ}=\right.$ Streptogaster papillatus N. A. Cobb 1898),

Heth dimorphum M. B. Chitwood 1935,

Heth hexaspinosum M. B. Chitwood 1935,

b) Deux espèces dont on connaît seulement la $q$ :

Heth spinosum P. Artigas 1930,

Heth duvidosum P. Artigas 1930, 
c) Deux espèces dont on connaît seulement le $\delta$ :

Heth sp. (I) P. Artigas 1930,

Heth sp. (II) P. Artigas 1930.

Les Heth-Streptogaster que j'ai examinés diffèrent des espèces ci-dessus.

\section{HETH TUZET $Æ$ n. sp.}

(Fig. 57-58)

․ La tête porte antérieurement 2 paires de languettes striées transversalement et 2 paires de protubérances en forme de bâtonnets arqués. Le bord apical de la tête, occupé par les deux lèvres buccales, est strié. Un collier d'épines triangulaires aiguës fait suite à la tête. Du côté droit et du côté gauche se trouvent 3 paires d'expansions cuticulaires dirigées postérieurement ; la paire antérieure est une lame se terminant par deux pointes postérieures ; la seconde paire, un peu en arrière de la première, comprend 4 épines incurvées, dont les bases sont contiguës deux par deux ; la troisième paire, un peu en arrière de la deuxième, comprend deux lames juxtaposées, terminées chacune par deux pointes. Je n'ai pas vu d'autres ornements cuticulaires. Le corpus œsophagien est cylindrique, il est suivi par un isthme de même diamètre, qui est un prolongement du bulbe; il y a une ligne de démarcation entre le corpus et l'isthme, il n'y en a pas entre l'isthme et le bulbe. Le bulbe contient un appareil malaxeur qui ne se continue pas dans le début de l'intestin. L'anus est à une petite distance en arrière de l'orifice f. Le vagin est dirigé antérieurement, les œufs utérins sont peu nombreux, environ 4 à 10 , et très gros, mesurant $182 \times 107,187 \times 105,188 \times 110 \mu$.

\section{Dimensions :}

Longueur totale ................... 2.605

$\begin{array}{lll}\text { Largeur au niveau de l'œsophage } \ldots \ldots \ldots & 0.125\end{array}$

Largeur maximum .................. 0.274

Longueur de la queue ............... 0.580

Distance de la vulve à l'anus $\ldots \ldots \ldots \ldots \ldots .6 .125$

$\begin{array}{lll}\text { Longueur du corpus œsophagien ......... } & 0.223\end{array}$

Largeur du corpus œsophagien ......... 0.025

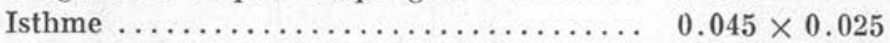

Bulbe, diamètre $\ldots \ldots \ldots \ldots \ldots \ldots \ldots \ldots . . .6 .100 \times 0.100$

Provenance : Intestin de Psendonannolene tricolor $\mathrm{H}$. W. Brölemann, Etat de São-Paulo, 1886 et 1888. 

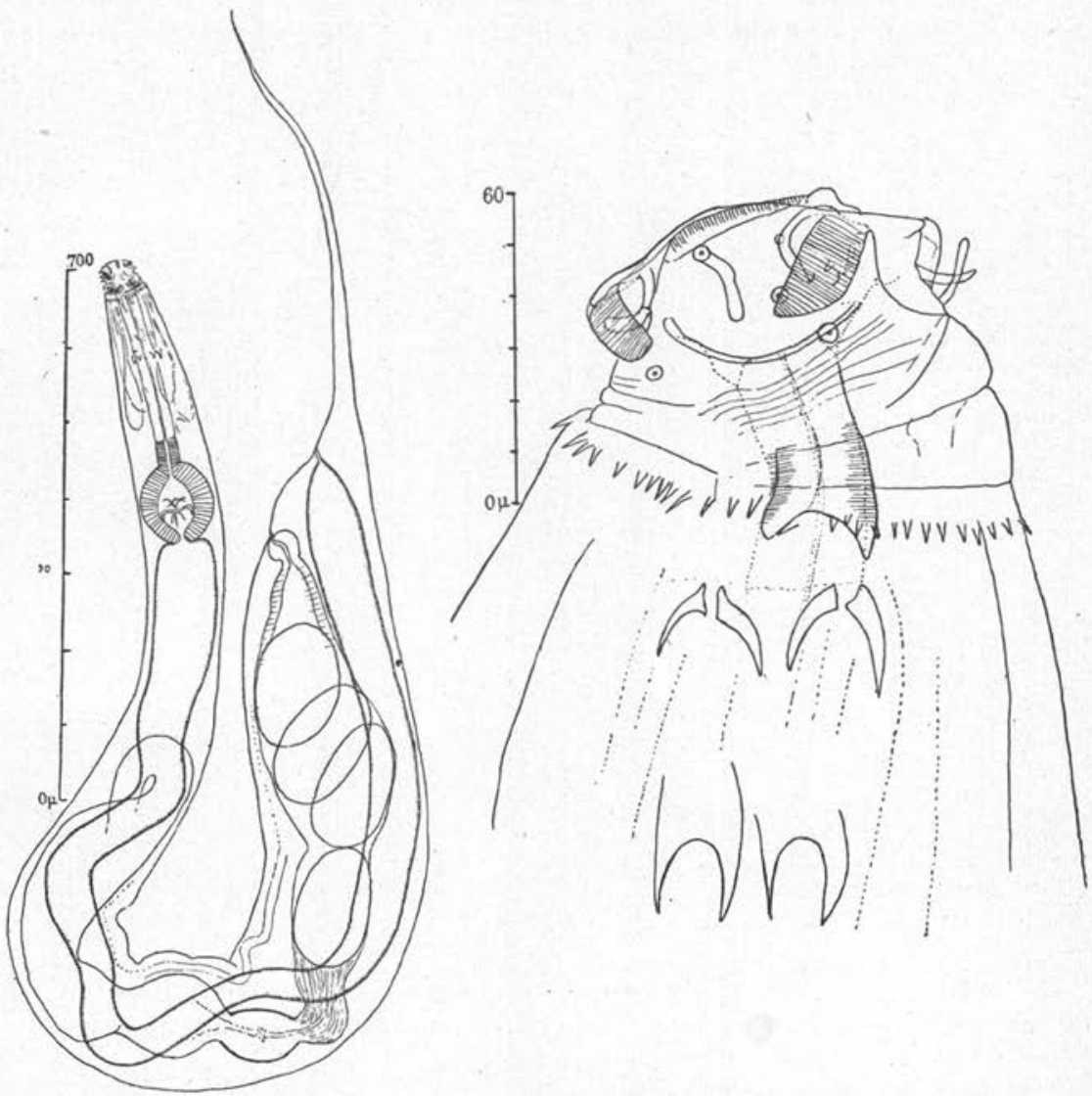

FIG. 57. - Heth Tuzete n. sp. Intestin de Pseudonannolene tricolor H. W. Brölemann. Pirassununga (Etat de São Paulo, Brésil). Odette Tuzet ded. \& à maturité.

Fig. 58. - Même espèce et même provenance que pour la figure 57 . Extrémité antérieure d'une ?.

\section{HETH TRAVFILHOI (1) n. sp.}

(Fig. 59-60)

․ Tête bien délimitée, terminée du côté droit et du côté gauche par deux lobes latéraux un peu globuleux, portant chacun une grosse papille; dans l'espace entre les deux lobes s'ouvre une amphide. A une très petite distance en arrière des lobes terminaux se trouve une cein-

(1) Cette espèce est dédiée au $\mathrm{D}^{\mathrm{r}}$ Lauro Travassos filho, qui a eu l'amabilité de me procurer la thèse de Paulo Artigas. 


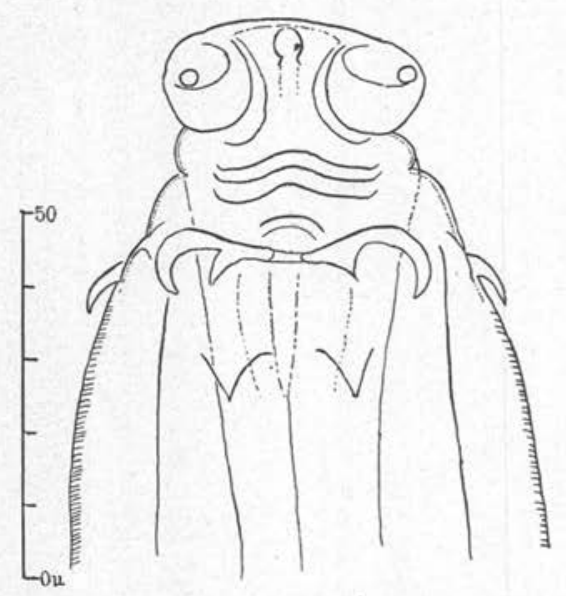

Fig. 59. - Heth Travfilhoi n.-sp. Intestin de Rhinocricus padbergi Verhoeff. Etat de São Paulo (Brésil), 1902. Odette Tuzet ded. Extrémité antérieure d'une $\$$.

FIG, 60. - Même espèce et même provenance que pour la figure 59. Région postérieure d'une $\leftrightarrow$.

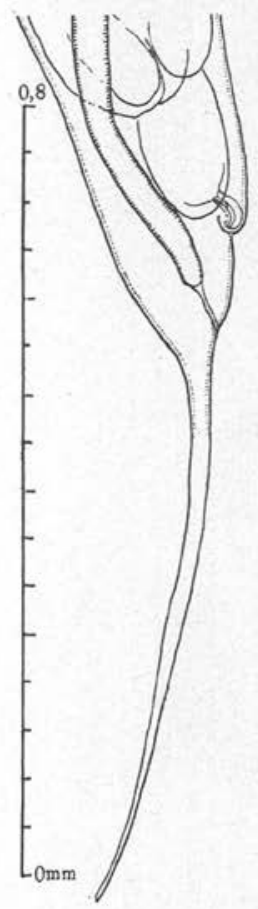

ture de grands crochets ; une paire, sur chaque face, et deux paires, sur chaque côté; les deux d'une même paire, sur les côtés, ont une base transversale commune. Plus postérieurement, il y a, sur chacun des côtés, deux crochets ou lames triangulaires, séparés l'un de l'autre par un petit espace. L'œsophage est subcylindrique, plutôt grêle, il se continue par l'isthme, avec délimitation marquée par une constriction; l'isthme est en continuité avec le bulbe contenant un puissant appareil masticateur. L'intestin décrit des sinuosités et s'ouvre très peu en avant du rétrécissement caudal donnant naissance à la longue queue filiforme. La vulve est à une faible distance en avant de l'anus. J'ai compté 6 à 8 œufs utérins, mesurant environ $156 \times 95,167 \times 95,177 \times 100 \mu$.

Dimensions (en mm.), d'une $q$ ovigère :

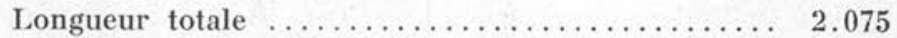

Epaisseur de la tête ................. 0.040

Plus grande largeur du corps $\ldots \ldots \ldots \ldots \ldots \ldots \ldots . \ldots .230$

Distance de l'extrémité antérieure à la vulve $\ldots \ldots \quad 1.460$

Distance de la vulve à l'anus ............. 0.070

Queue ........................ 0.545 
Corpus œsophagien, longueur $\ldots \ldots \ldots \ldots \ldots \ldots \ldots \quad 0.228$

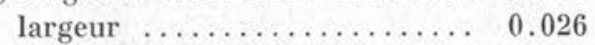

Isthme œsophagien, longueur $\ldots \ldots \ldots \ldots \ldots \ldots \ldots \quad 0.045$

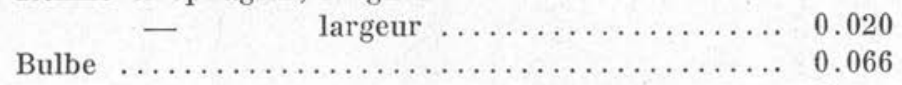

Provenance : Intestin de Rhinocricus Padbergi Verhoeff. Etat de SãoPaulo, 1902.

\section{HETH TRAVASSOSI n. sp.}

(Fig. 61-63)

ๆ. Tête bien délimitée, terminée du côté droit et du côté gauche par 2 lobes ovales à striation transversale ; entre ces lobes, sur chacun des côtés de la tête, s'ouvre une amphide; au même niveau, sur chaque face, il y a deux grosses papilles. La limite postérieure de la tête présente, sur chacune des faces, deux grandes épines triangulaires divergentes, et sur chacun des côtés une petite lame transversale, dont chaque côté se prolonge par une épine. Immédiatement au-dessous, au milieu du côté droit, comme du côté gauche, il y a une succession de trois autres lames, un peu trapézoïdes, dont les bords latéraux se prolongent par une épine postérieure. Le corpus œsophagien est subcylindrique et se continue par l'isthme, dont il est bien délimité par une constriction. Tout à fait postérieurement, au voisinage de l'isthme, le corpus œsophagien semble avoir une structure musculaire un peu différente. L'isthme (goulot du bulbe) est court, le bulbe contient un puissant appareil masticateur. L'intestin décrit quelques sinuosités et s'ouvre loin en arrière de la vulve. A partir de la vulve, le corps s'atténue lentement, pour se terminer par une queue filiforme. L'utérus acquiert un grand développement et peut contenir environ 25-30 œufs, mesurant environ 61-65 $\times 120 \mu$, à $75 \times 110 \mu$; quelques-uns, plus petits, mesurent seulement $50 \times 95 \mu$.

\section{Dimensions, en mm. :}

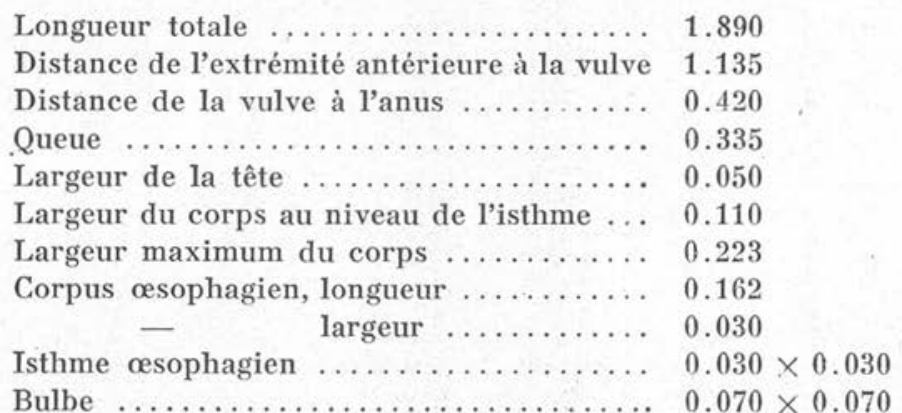

Provenance : Intestin de Leptodesmus jucundus H. W. Brölemann. Environs de Descalvado-Pirassununga (Etat de São-Paulo). 

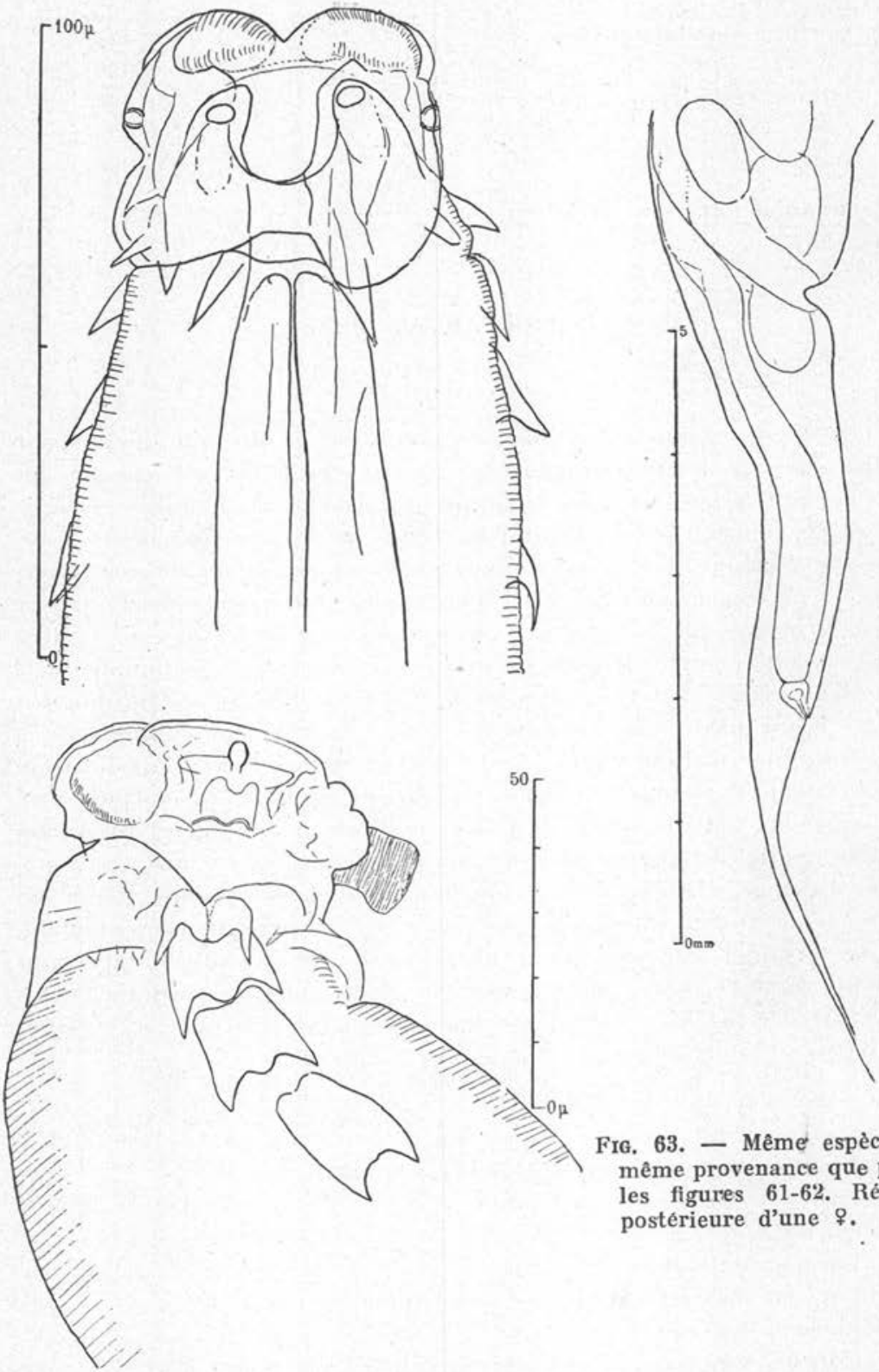

FrG, 63. - Mêmé espèce et même provenance que pour les figures 61-62. Région postérieure d'une ?.

Fig. 61. - Heth Travassosi n. sp. Intestin de Leptodesmus jucundus. H. W. Brölemann. Environs de Descalvado-Pirassununga (Etat de São Paulo, Brésil). Odette Tuzet ded. Extrémité antérieure d'une $\%$, vue par une face.

Fıg. 62. - Même espèce et même provenance que pour la figure 61 . Extrémité antérieure d'une 


\section{HETH ARTIGASI n. sp.}

(Fig. 64-65)

La tête est presque tronquée à l'apex, qui est large, à peu près aussi large que le corps en arrière de la tète. Après le plateau apical, la tête
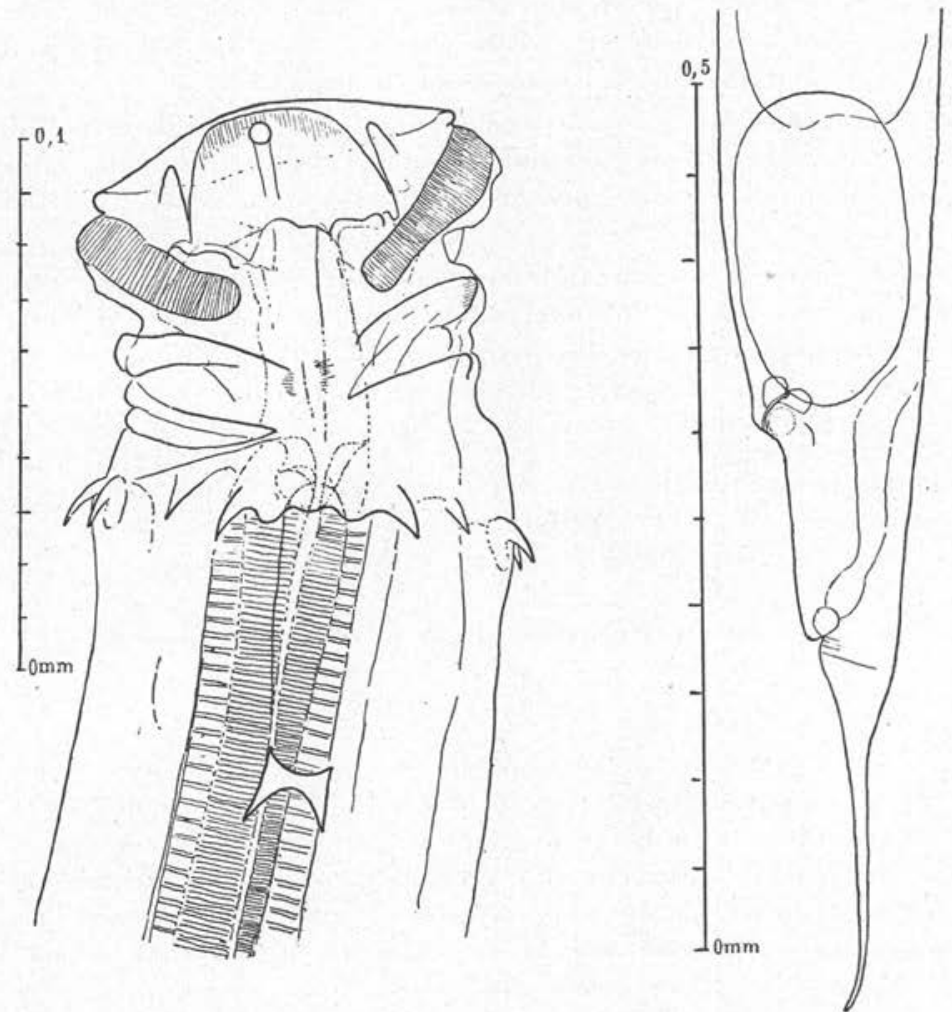

Fig. 64. - Heth Artigasi n.sp. Intestin de Rhinocricus sp., $\mathrm{n}^{\circ} 3.320$. Environs d'Episacio (Etat de São Paulo, Brésil). Odette Tuzet ded. Extrémité antérieure d'une \&, vue latérale.

Fig. 65. - Même espèce et même provenance que pour la fig. 64 . Région postérieure d'une + .

s'élargit un peu et présente, sur chacun des côtés, une paire de languettes striées transversalement, dirigées obliquement de dehors en dedans, mais les deux d'un même côté sont loin d'arriver au contact l'une de l'autre. Au-dessous, se trouvent, sur chaque face, 2 bourrelets étagés, s'étendant un peu sur les côtés. A la limite inférieure de la tête, 
il y a une rangée transversale de quelques fortes épines recourbées; les deux les plus rapprochées du milieu d'un côté sont réunies par un rebord ondulé. Beaucoup en arrière de ces épines, mais à une distance inférieure à la longueur de la tête, se trouve, au milieu de cinacun des côtés, une pièce en forme de 2 triangles, réunis par un isthme à bords antérieur et postérieur concaves. La cavité bucco-pharyngienne s'étend dans toute la longueur de la tête; le corpus œsophagien est subcylindrique, il est continué, avec démarcation, par un prolongement, légèrement élargi antérieurement, du bulbe ; celui-ci contient un appareil masticateur ne s'étendant pas dans le début de l'intestin.

Un peu avant la vulve, le corps s'atténue lentement en direction postérieure, pour se terminer par une queue presque filiforme, plus longue que la distance entre la vulve et l'anus. Les œufs sont gros et en très petit nombre.

Le spécimen type est long de $1,51 \mathrm{~mm}$., dont 0,22 pour la queue, avec une largeur de 0,07 à 0,15 , la vulve est distante de 0,34 de l'extrémité caudale; le corpus wosophagien est long de 0,218 , large de 0,30 , l'isthme est long de 0,05 , le bulbe mesure $0,076 \times 0,076$. Il y a 3 oufs utérins mesurant $152-170 \times 100 \mu$.

Provenance : Intestin de Rhinocricus sp., des environs d'Episacio (Etat de São-Paulo); $\mathrm{n}^{\circ} 3320 ; 22-1-1950$.

\section{HETH PERARMATUM n. sp.}

(Fig. 66-67)

ㅇ. La tête porte antérieurement 2 paires de languettes striées transversalement; un peu au-dessous se trouvent, de chaque côté, deux lamelles à bord libre arrondi, garni d'épines; un peu plus bas il y a, de chaque côté, encore deux lamelles à bord libre arrondi garni d'épines; à partir de ce niveau, et sur une petite hauteur, la cuticule présente de minuscules aspérités, disposées suivant des lignes transversales. Un collier d'épines avec, au même niveau, quelques épines plus grosses, limite postérieurement la tête ; il est interrompu, sur chacun des côtés, par une pièce formée de deux triangles réunis par un de leurs sommets; un angle aigu de chaque triangle est dirigé postérieurement, formant une grande épine; au-dessous se trouve une paire de grandes épines triangulaires, soudées par un angle de leur base; plus bas se trouve encore une paire de grandes épines triangulaires, se touchant par un angle de leur base, mais ne paraissant pas unies l'une à l'autre. Entre le niveau du collier de petites épines et le niveau de l'extrémité des grandes épines postérieures, le tégument porte quelques épines dispersées et de très petites plaques arrondies, dont le bord libre est pourvu de quelques très petites épines. L'œsophage, subcylindrique, est long et grêle ; il est continué par un isthme, qui est un prolongement du bulbe. 
Le bulbe contient un appareil masticateur ne se continuant pas dans le début de l'intestin. L'anus est situé très peu en avant du rétrécissement caudal. La queue, plutôt longue, s'atténue en pointe fine.

Les œufs utérins, assez gros, mesurent $120 \times 90$, à $125 \times 100 \mu$, leur nombre dépasse peu une vingtaine.

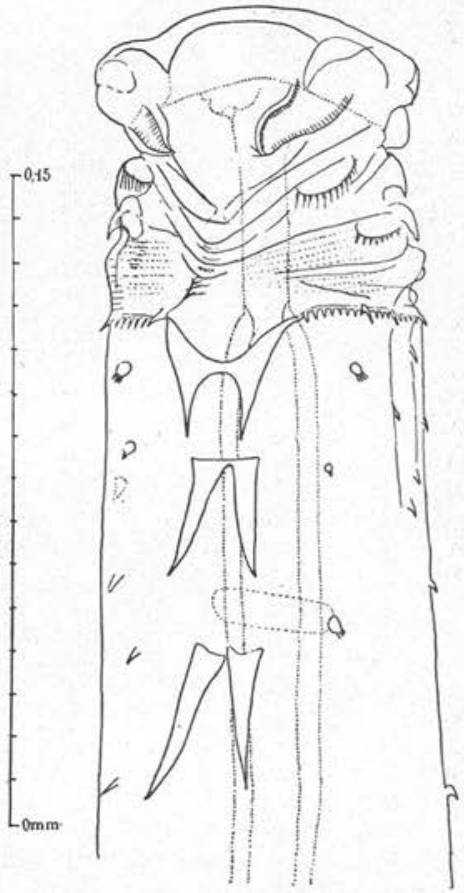

FIg. 66. - Heth perarmatum n. sp. Intestin de Rhinocricus cachoeirensis O. Schubart. Etat de São Paulo (Brésil), 1900. Odette Tuzet ded. Extrémité antérieure d'une ?.

FIG. 67. - Même espèce et même provenance que pour la fig. $66 . \quad \Varangle$ à maturité.

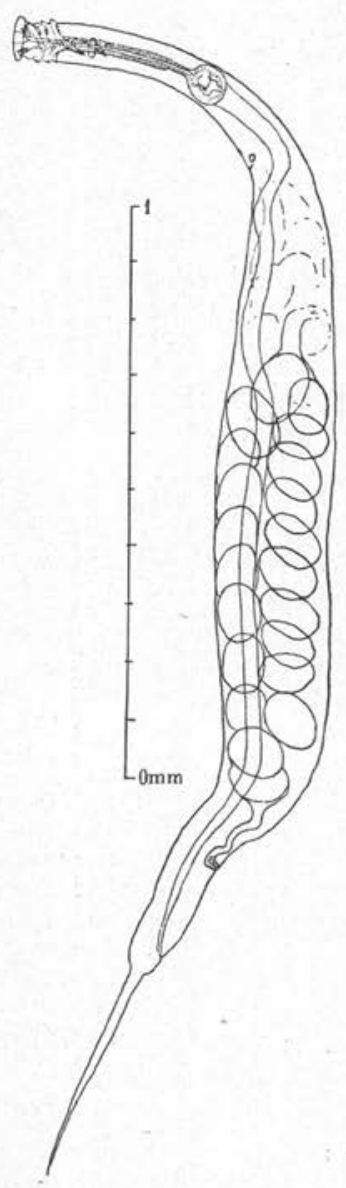

Une $q$ à maturité est longue de $2,80 \mathrm{~mm}$. (dont 0,43 pour la queue), large de 0,34 ; le corpus œsophagien + isthme est long de 0,279 , large de 0,025 ; le bulbe mesure $0,090 \times 0,100$; la vulve est à 0,167 , en avant de l'anus.

Le ${ }^{\star}$ n'est pas connu.

Parmi les espèces de Heth jusqu'à présent décrites, duvidosum est très voisine de perarmatum; mais les deux paires de languettes striées trans- 
versalement de la partie antérieure de la tète, bien apparentes chez perarmatum, n'existent pas chez duvidosum.

Provenance : Intestin de Rhinocricus cachoeirensis O. Schubart. Etat de São-Paulo ; 1900.

Quelques individus portent des Eccrinides (fig. 68).

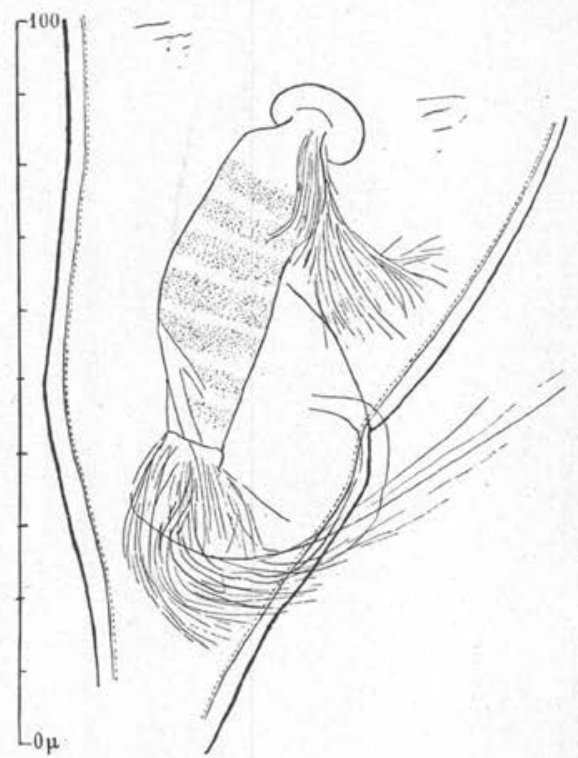

Fig. 68. - Eccrinide fixée un peu en avant de l'anus d'une $\subsetneq$ de Heth perarmatum mihi, de Rhinocricus cachoeirensis O. Schubart. Etat de São Paulo (Brésil), 1900. Odette Tuzet ded. Il s'agit apparemment d'un filament âgé, à la fin de son évolution, il porte des bouquets de bactéries.

\section{HETH SCHUBARTI n. sp.}

(Fig. 69-70)

․ A une petite distance en arrière de l'apex, la tête porte, sur chacun de ses côtés, une paire de languettes striées transversalement. Ces languettes sont orientées en direction transversale, leur extrémité vers la mi-largeur d'un côté de la tête. Au-dessus de chaque languette se trouve un gros crochet. A la limite inférieure de la tête, il y a une rangée d'épines un peu espacées, formant collier. Beaucoup plus loin, à une distance du collier un peu supérieure à la distance entre le collier et l'apex, il y a deux fortes épines à la face dorsale comme à la face ventrale. La cuticule ne porte pas d'ornements sur le reste du corps. L'œso- 
phage, cylindriforme, est suivi par un isthme un peu renflé antérieurement, puis un peu rétréci, qui est un prolongement du bulbe; sans démarcation avec le bulbe. Dans le bulbe, il y a un appareil malaxeur puissant, ne se continuant pas dans le début de l'intestin. L'intestin décrit quelques boucles ou sinuosités. La queue est longue et subulée, sans brusque rétrécissement près de son origine.

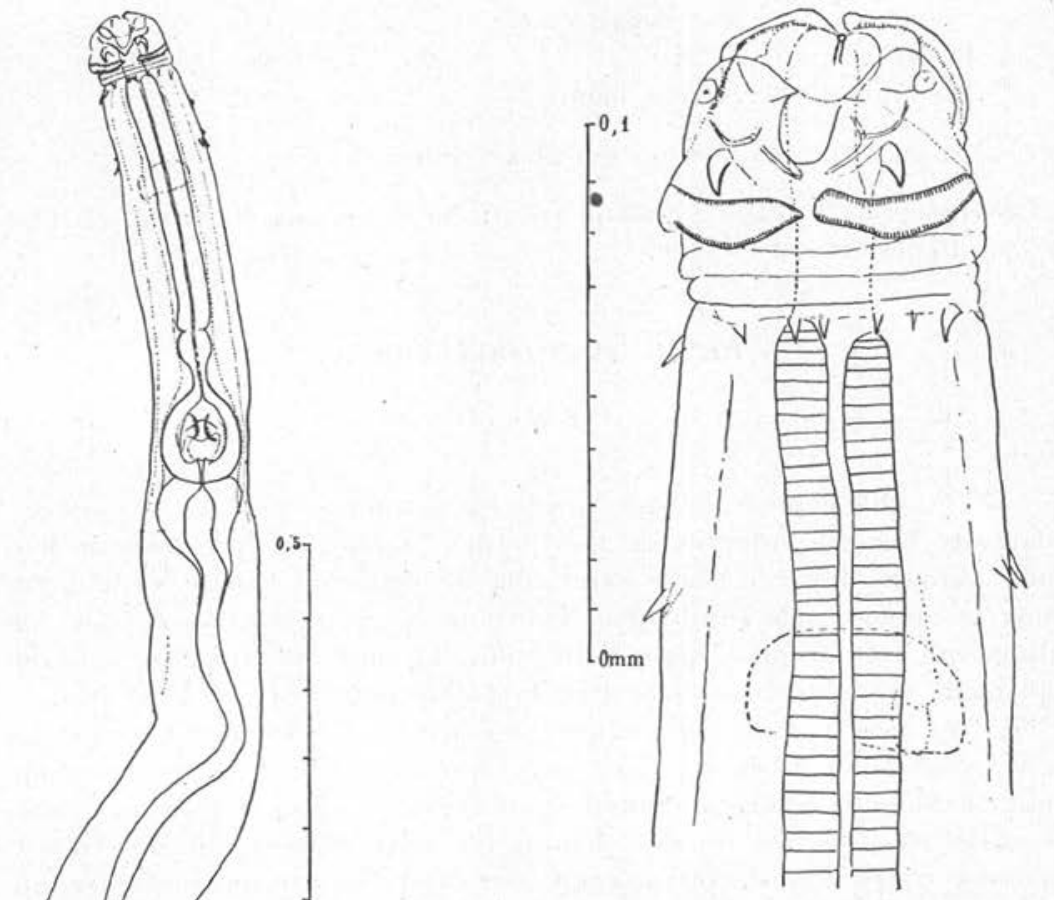

Fig. 69. - Heth Schubarti n. sp. Intestin de Rhinocricus cachoeirensis 0. Schubart. Etat de São Paulo (Brésil), 1900. Odette Tuzet ded.

FiG. 70. - Même espèce et même provenance que pour la fig. 69 . $q$ immature. 
Dimensions, en mm., d'une $q$ immature :

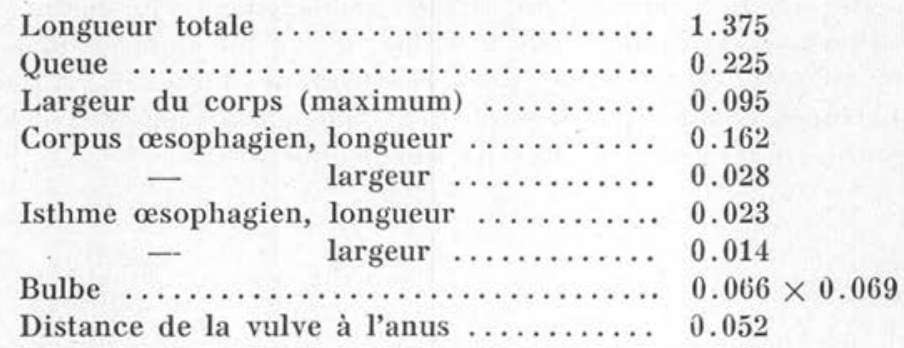

Je n'ai eu à ma disposition que des $q$ immatures.

Provenance : Intestin de Rhinocricus cachoeirensis O. Schubart. Etat de São-Paulo (Brésil) ; 1900.

\section{HETH-STREPTOGASTER A}

(Fig. 71-72)

ð. Extrémité céphalique brusquement arrondie, presque tronquée; ouverture buccale entourée de très petites papilles; cavité buccale longue, montrant dans sa moitié antérieure une suite de 4 ou 5 renflements. Long œsophage subcylindrique, continué avec démarcation par un isthme qui est une prolongation du bulbe. Le bulbe contient un appareil malaxeur, qui ne pénètre pas dans la partie antérieure de l'intestin.

Un très long spicule, peu arqué, à extrémité antérieure formant un petit crochet en direction ventrale. Un court et large gubernaculum, dont l'extrémité antérieure montre un court faisceau de petites protubérances en direction dorsale. L'anus est entre deux ventouses; il y a en outre une ventouse préanale un peu avant du niveau de l'extrémité antérieure du grand spicule. La queue est longue et subulée.

Pour un individu long de $1,405 \mathrm{~mm}$., dont 0,167 pour la queue, large de $0,05-0,09$, le corpus œsophagien est long de 0,376 , large de 0,014 , l'isthme est long d'environ 0,023 , large de 0,012 . Le bulbe (non compris son prolongement) mesure $0,052 \times 0,063$. Le spicule a une longueur de 0,150 , le gubernaculum une longueur de 0,043: Le centre de la ventouse préanale est à une distance de 0,153 en avant de l'anus.

Ce ô est très voisin du second de ceux décrits par P. Artigas (1929, p. 86), qui possède aussi, outre la ventouse, située assez loin en avant de l'anus, une petite ventouse préanale et une autre postanale.

Provenance : a) Intestin de Rhinocricus cachoeirensis O. Schubart. Etat de São-Paulo, 1900.

b) Intestin de Rhinocricus sp., environs d'Episacio (Etat de São-Paulo), $\mathrm{n}^{\circ} 3320 ; 22-1-1950$. 

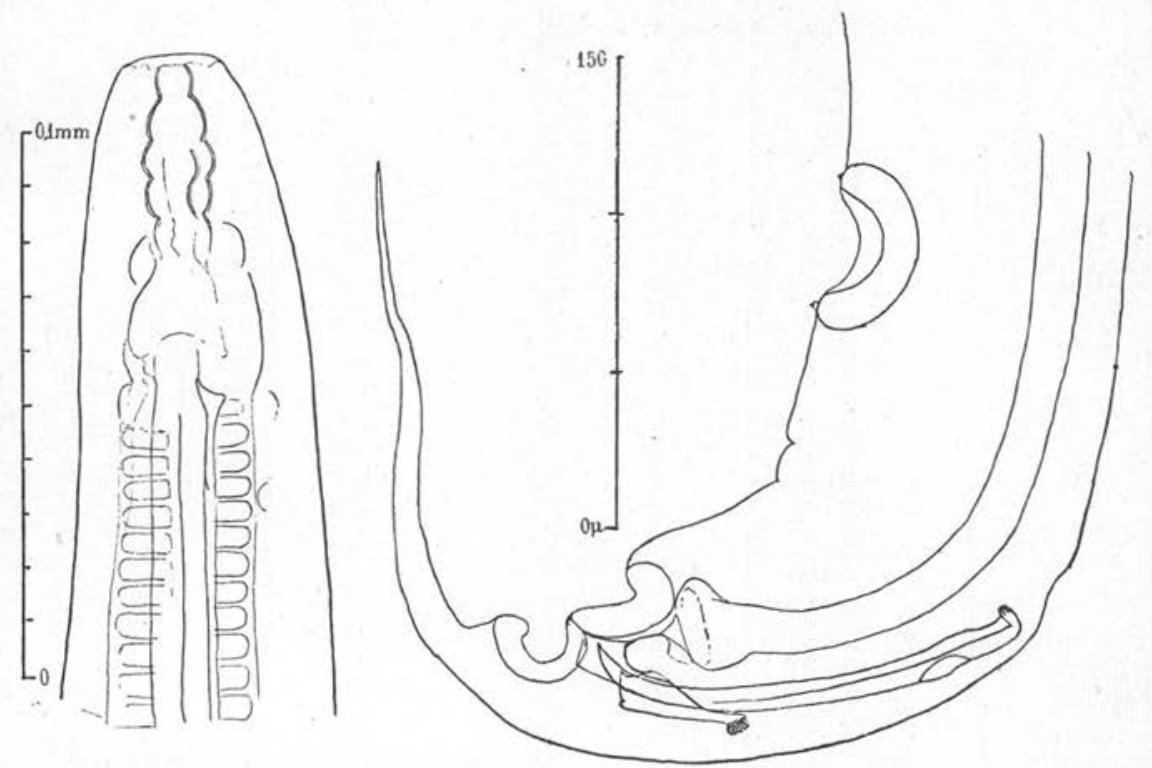

Fig. 71. - Heth-Streptogaster A. Intestin de Rhinocricus cachoeirensis O. Schubart. Etat de São Paulo (Brésil), 1900. Odette Tuzet ded. Région antérieure d'un $\sigma^{\circ}$.

FiG. 72, - Même espèce et même provenance que pour la figure 71. Région postérieure d'un $\delta$ long d'environ $1 \mathrm{~mm} .5$, large de $0 \mathrm{~mm}$. 05-0 mm. 09 .

\section{HETH-STREPTOGASTER B}

(Fig. 73-75)

ð. Extrémité antérieure subtronquée, terminée par 4 lobes peu accentués. Cavité buccale longue et probablement protractile, avec de légères dilatations étagées. Long corpus œsophagien subcylindrique, paraissant avoir une structure un peu différente au voisinage de son extrémité postérieure, se continuant avec démarcation, par un isthme qui est un prolongement du bulbe ; ce prolongement bulbaire est légèrement renflé antérieurement. Le bulbe contient un appareil masticateur ne pénétrant pas dans le début de l'intestin. L'anus est situé un peu en avant de la partie rétrécie de la queue; celle-ci se termine par une pointe plutôt courte (proportionnellement moins longue que chez Heth I et Heth II, de P. Artigas, et beaucoup moins longue que chez $H$. dimorphum M. B. Chitwood et $H$. hexaspinosum M. B. Chitwood). Il y a une ventouse préanale très loin en avant de l'anus.

Les deux spicules sont très inégaux; le grand spicule, peu arqué, est courbé à son extrémité proximale en un court crochet orienté ventralement; le petit spicule, qui est peut-être un gubernaculum, est forte- 

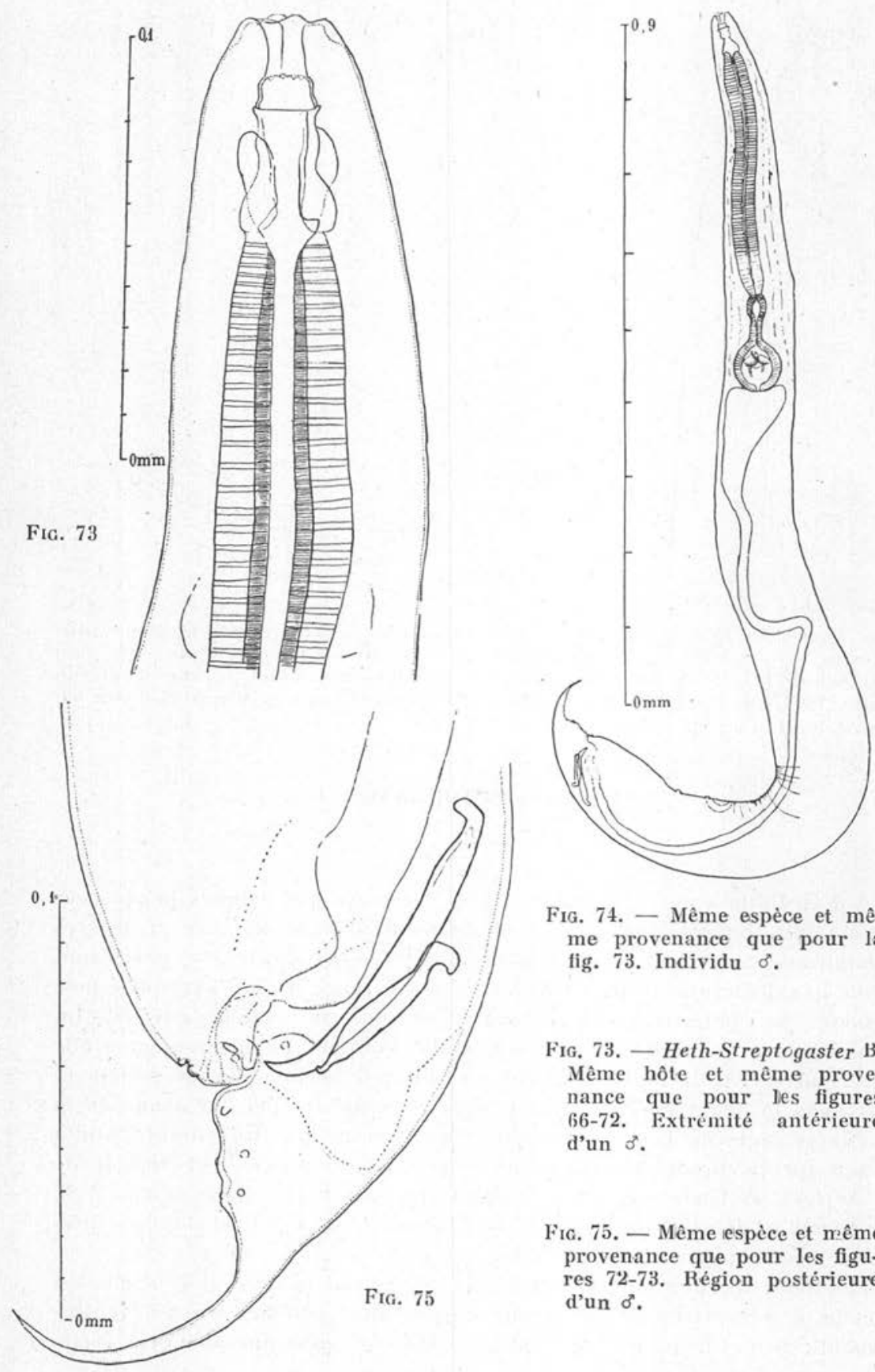

FIG. 74. - Même espèce et même provenance que pour la fig. 73. Individu $\delta$.

FIG. 73. - Heth-Streptogaster B. Même hôte et même provenance que pour les figures 66-72. Extrémité antérieure d'un $\sigma^{3}$.

Fic. 75. - Même iespèce et même provenance que pour les figures 72-73. Région postérieure d'un $\sigma^{\circ}$. 
ment recourbé à son extrémité proximale en un crochet orienté dorsalement. Il semble y avoir 3 paires de papilles postanales, 1 ou 2 paires adanales et au moins 2 paires préanales.

Pour un individu long de $1,486 \mathrm{~mm}$, dont 0,095 pour la queue, la largeur maximum est 0,135 ; le corpus œesophagien est long de 0,330 , large de 0,039 ; l'isthme est long de 0,050 , large de 0,022 ; le bulbe mesure $0,062 \times 0,062$, le grand spicule a une longueur de $152 \mu$, le petit de $75 \mu$.

Provenance : Intestin de Rhinocricus cachoeirensis O. Schubart. Etat de São-Paulo; 1900.

\section{HETH-STREPTOGASTER C}

(Fig. 76-77)

6. Extrémité antérieure régulièrement arrondie, avec papilles mal distinctes. Cavité buccale plus longue que large. Long œsophage subcylindrique, grêle ; isthme relativement court, bulbe avec puissant appareil masticateur. Intestin décrivant des sinuosités. Ventouse préanale assez loin en avant de l'anus, ayant son centre à une distance de l'anus à peu près égale à la distance de l'anus à l'extrémité caudale. Le spicule est peu incurvé ; son extrémité antérieure présente deux légères protubérances en crochet, ce qui peut faire croire à 2 spicules égaux soudés l'un à l'autre ; toutefois, comme il n'y a qu'une seule pointe postérieure, j'admets qu'il n'y a pas 2 spicules. Le gubernaculum est légèrement plus long que la mi-longueur du spicule. Queue se rétrécissant assez brusquement un peu au delà de l'anus et se terminant par un prolongement filiforme.

Dimensions, en mm. :

\begin{tabular}{|c|c|}
\hline & \\
\hline ....... & 0.218 \\
\hline ur maximum & 0.175 \\
\hline orpus + isthme & 0.442 \\
\hline ur du corpus $\ldots \ldots \ldots$ & 0.036 \\
\hline ( & 0.085 \\
\hline tre de la ventouse à l'anus .. & 0.218 \\
\hline ueur & 0.11 \\
\hline Im & 0.0 \\
\hline
\end{tabular}

Provenance : Intestin de Rhinocricus Padbergi Verhoeff ; Etat de SãoPaulo (Brésil).

L'unique individu à ma disposition est en médiocre état. Je suppose qu'il est le ơ de Heth travfilhoi mihi, parce que la seule $\uparrow$ trouvée en même temps est celle décrite plus haut sous ce nom. 

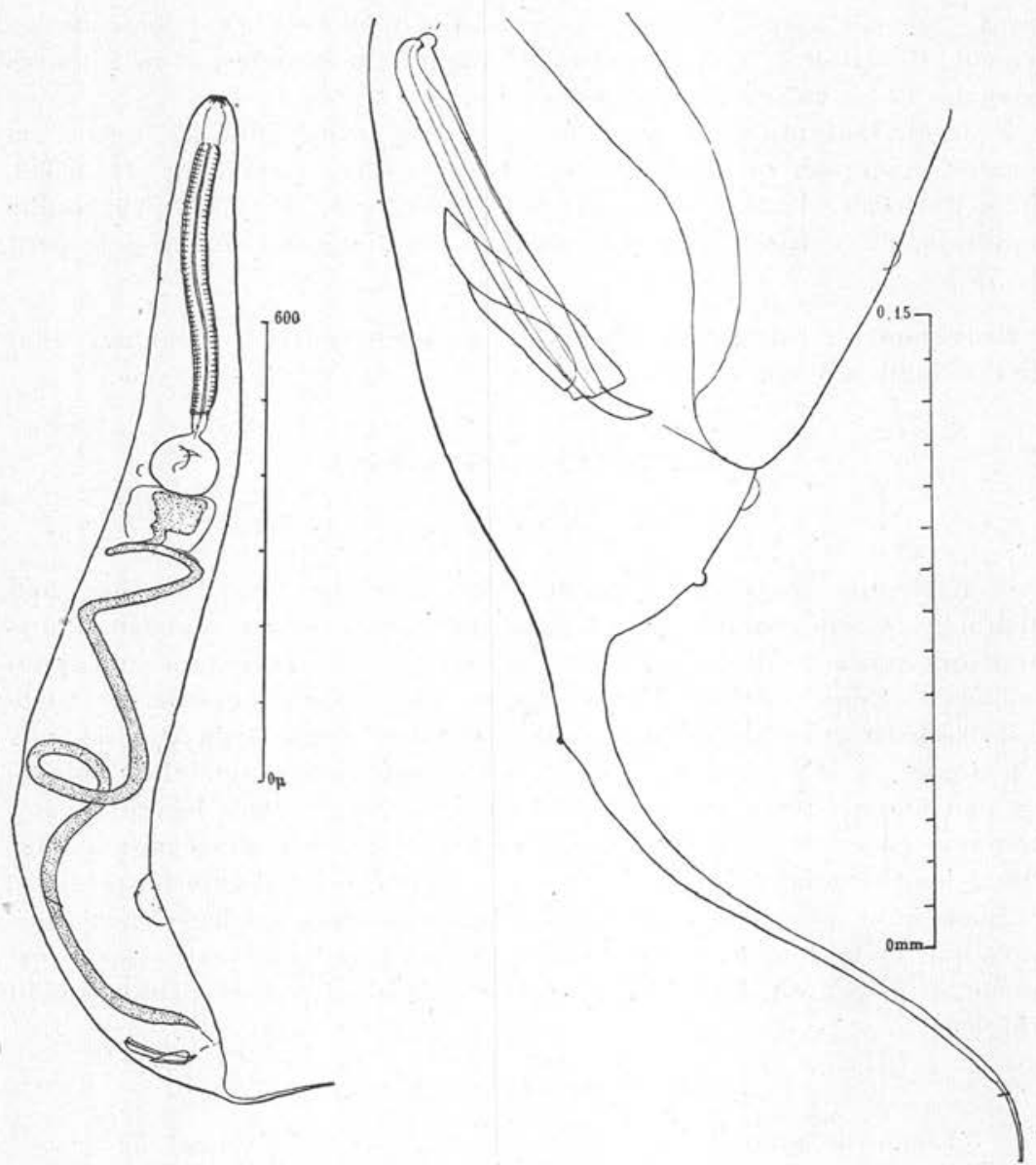

Fig. 76. - Heth-Streptogaster C. Intestin de Rhinocricus padbergi Verhoeff. Etat de São Paulo (Brésil), 1902. Odette Tuzet ded. Spécimen o".

FIG. 77. - Même espèce et même provenance que pour la figure 76 . Région postérieure d'un ơ long d'environ $1 \mathrm{~mm} .52$.

\section{HETH-STREPTOGASTER D}

(Fig. 78-79)

Je désigne sous ce nom provisoire quelques spécimens $\widehat{o}$ présentant une très grande ressemblance avec l'espèce précédente, mais qui sont notablement plus petits et ont un prolongement caudal proportionnellement beaucoup plus court. 


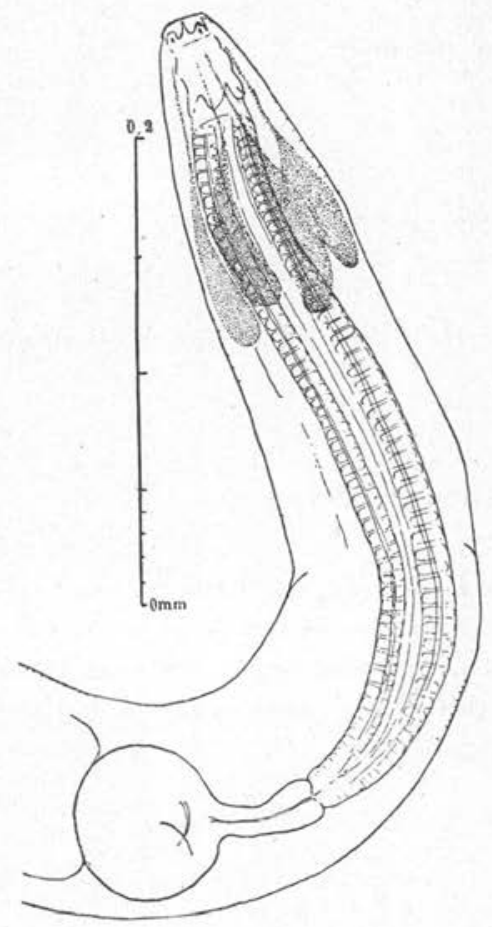

FiG. 78. - Heth-Streptogaster D. Intestin de Rhinocricus sp., des environs d'Episacio (Etat de Sảo Paulo, Brésil), $\mathrm{n}^{\circ} 3.320,22-1-1950$. Odette Tuzet ded.

Fig. 79. - Même espèce et même provenance que pour la figure 78 . Région postérieure d'un ơ long d'environ $1 \mathrm{~mm} .14$.

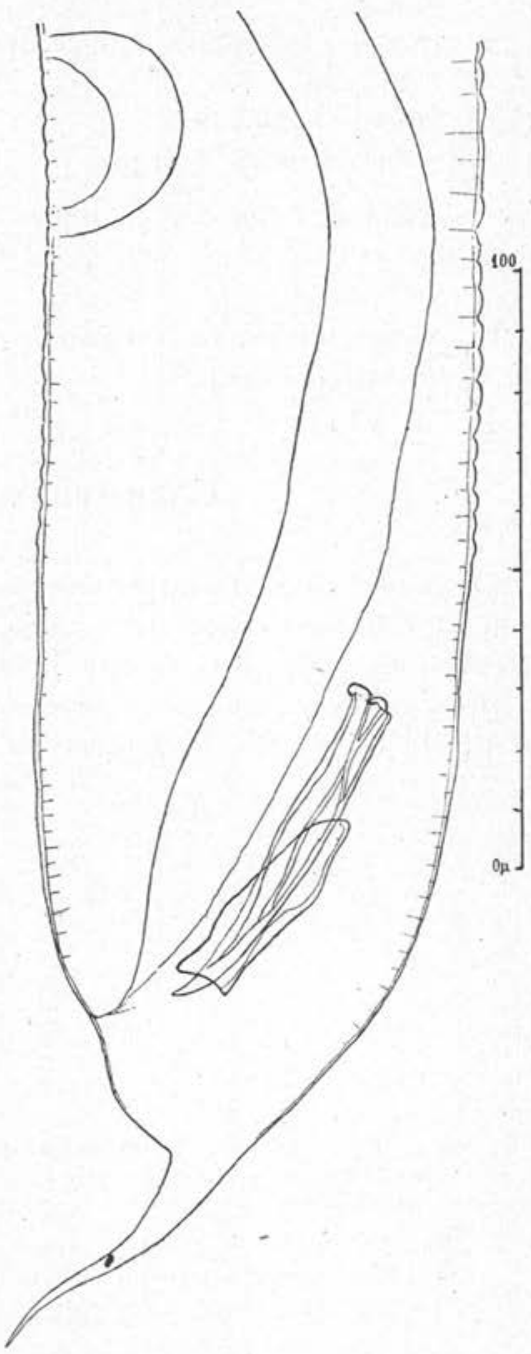

Dimensions (en mm.) :

Longueur totale ................ 1.140

Largeur maximum ............. 0.090

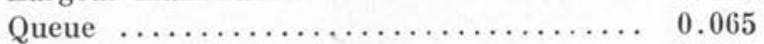

Corpus œsophagien, longueur ........ 0.315

largeur $\ldots \ldots \ldots \ldots \ldots 0.045$

Isthme, longueur $\ldots \ldots \ldots \ldots \ldots \ldots \ldots \ldots . \ldots \ldots 45$

- largeur ................ 0.015 à 0.020 


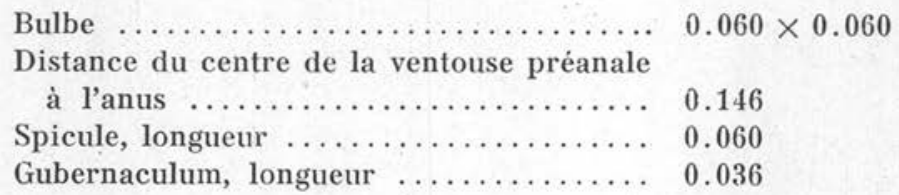

Provenance : Intestin de Rhinocricus sp., des environs d'Episacio (Etat de São-Paulo); n $3320 ; 22-1-1950$.

En même temps, se trouvaient des Heth-Streptogaster A. et des $q$ de Heth Artigasi mihi.

\section{Carnoya G. Gilson 1898}

Ce genre, où le dimorphisme sexuel est très accentué, est actuellement représenté par deux espèces dont les deux sexes ont été décrits : C. vitiensis G. Gilson 1898 et $C$. pyramboya P. Artigas 1926. Il ne semble pas que, depuis leur publication originale, ces espèces aient été redécrites ou à nouveau signalées.

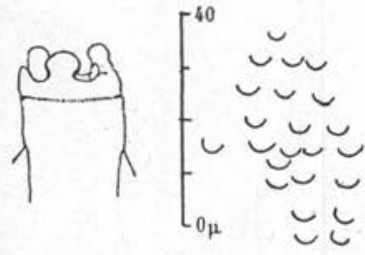

FIG. 80

FiG. 81

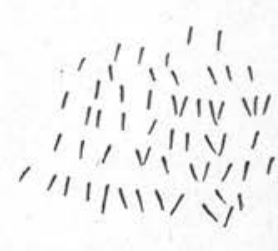

FiG. 82

Fig. 80. - Carnoya (gen. ?) dubia n. sp. Intestin de Rhinocricus sp., $\mathrm{n}^{\circ} 3.320$. Environs d'Episacio (Etat de São Paulo, Brésil), 22-1-1950. Odette Tuzet ded. Extrémité antérieure d'un $\delta$.

FiG. 81. - Même espèce et même provenance que pour la figure 80 . Ornementation cuticulaire de la face dorsale.

FIG. 82. - Même spécimen que pour les figures 80-81. Ornementation cuticulaire de la face ventrale.

\section{CARNOYA (gen. ?) DUBIA n. sp.}

(Fig. 80-84)

ð. Extrémité antérieure atténuée, avec tête distincte, se terminant par 3 lobes proéminents, arrondis, chacun d'eux étant bien séparé de ses voisins par un sinus. Cavité bucco-pharyngienne longue et un peu rétrécie en son milieu. Au niveau de la partie antérieure du corpus œsophagien, le tégument est orné, à la face dorsale, de très petites écailles 


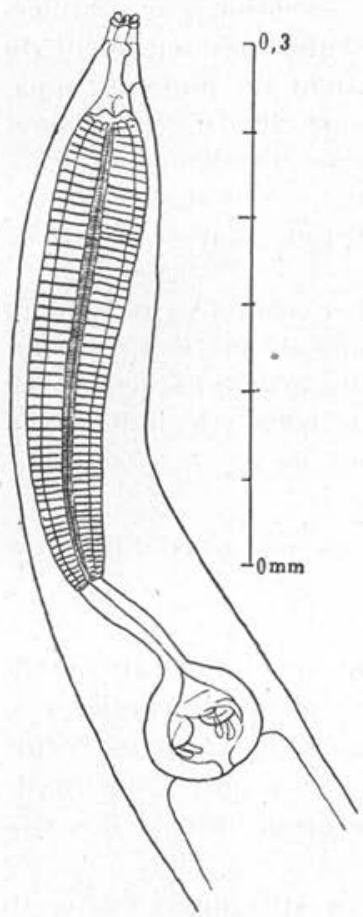

FIG. 83. - Même spécimen que pour les figures 80-82. Région antérieure.

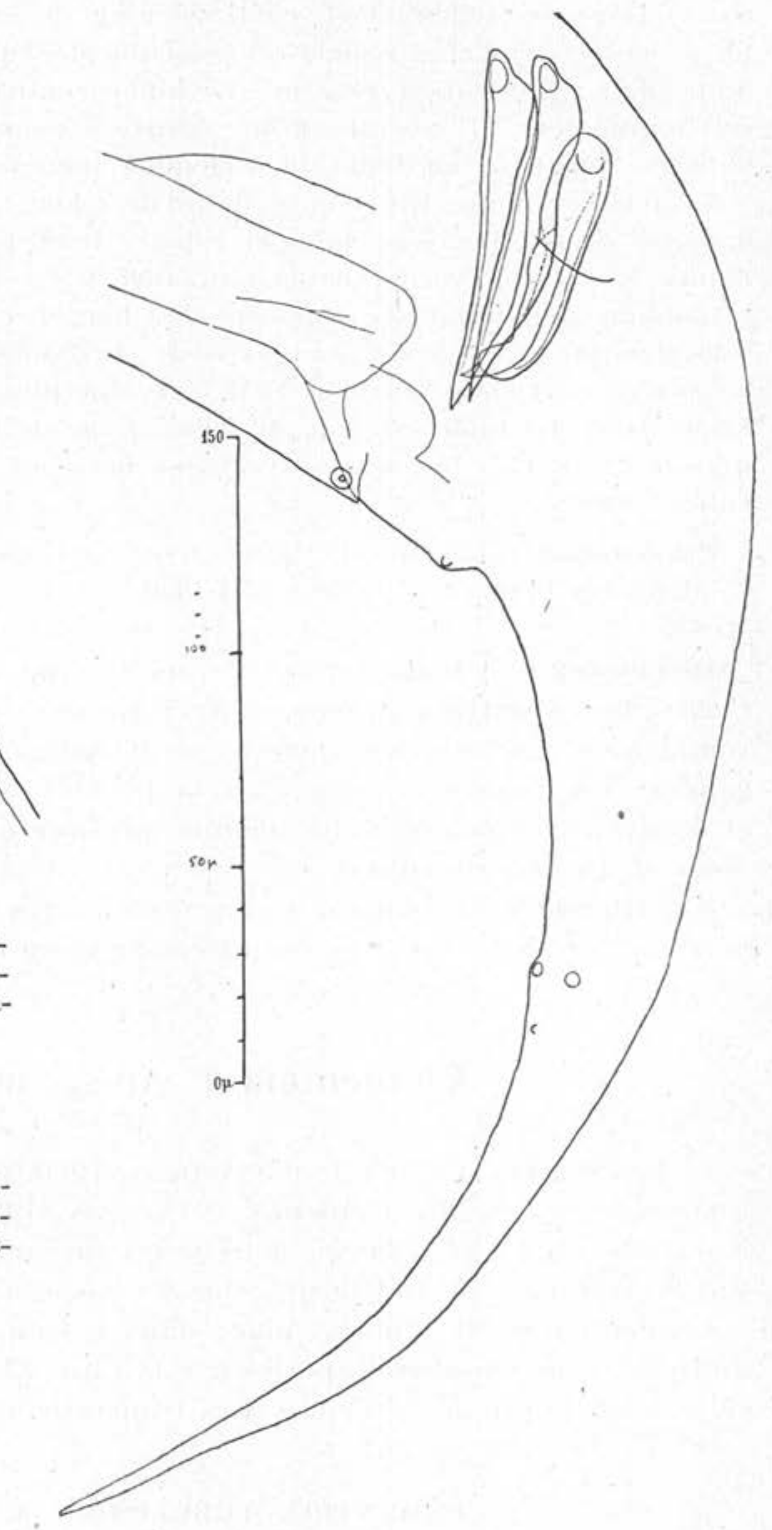

Fig, 84, - Même spécimen que pour les figures 80-83. Région postérieure.

demi-circulaires, larges d'environ $4 \mu$, disposées en rangées tranversales irrégulières (fig. 81) ; à la face ventrale, de très petites (longueur environ $4 \mu$ ) et fines épines en rangées transversales (fig. 82). Corpus œsophagien long, plutôt fusiforme que subcylindriforme, devenant un peu 
moins large postérieurement, continué avec démarcation par l'isthme, un peu plus étroit. Cet isthme est le goulot du bulbe, prolongement du bulbe sans démarcation avec lui. Le bulbe contient un puissant appareil masticateur. Il y a 2 spicules égaux, presque droits, s'élargissant antérieurement, et un long gubernaculum incurvé distalement.

La queue s'atténue lentement, elle est très longue, subfiliforme. Il y a 3 paires de papilles postanales et 1 paire immédiatement en avant de l'anus. Je n'ai pas vu de ventouse préanale.

L'unique individu à ma disposition est long d'environ $1,15 \mathrm{~mm}$. (dont 0,30 pour la queue), avec une épaisseur maximum de 0,110 . Le corpus œsophagien est long d'environ 0,258 , avec une plus grande largeur d'environ 0,056 . L'isthme est long de 0,065 , large de 0,020 ; le bulbe a un diamètre de 0,065 . Les spicules ont une longueur de $84 \mu$, le gubernaculum de $62 \mu$.

Provenance : Intestin de Rhinocricus sp., des environs d'Episacio (Etat de São-Paulo) ; $\mathrm{n}^{\circ} 3320 ; 22-1-1950$.

Discussion. - L'attribution de cet unique spécimen au genre Carnoya est incertaine ; néanmoins, il présente d'assez grandes ressemblances avec la $q$ de $C$. pyramboia P. Artigas 1926 : même forme générale du corps avec longue queue portant des papilles, profonde et étroité cavité bucco-pharyngienne, presque même forme des spicules et du gubernaculum.

En attendant de pouvoir examiner d'autres spécimens en meilleur état, je laisse provisoirement cette espèce dans Carnoya.

\section{Clementeia P. Artigas 1930}

Ce genre a été créé par Paulo Artigas (1930, p. 32, diagnose) pour une seule espèce : Cl. clementei P. Artigas (1930, p. 32-34, pl. XV, fig. 1,3 ; $2,4-7$ ठ), dont les deux sexes furent trouvés dans l'intestin d'un Myriapode indéterminé de Rio-de-Janeiro (Brésil).

Clementeia a été d'abord placé dans la sous-famille Ransomnematinæ, supposée alors appartenir à la fam. Thelastomatidæ ; mais cette sous-famille a été depuis lors transférée dans les Atractidæ.

\section{CLEMENTEIA TUBULIFERA n. $\mathbf{s p .}$}

(Fig. 85-90)

§. Cette espèce est très voisine du générotype ; comme chez celui-ci, le bord de l'orifice buccal montre deux protubérances en forme de cornes, mais il n’y a pas, entre la bouche et l'œsophage, la « série de 

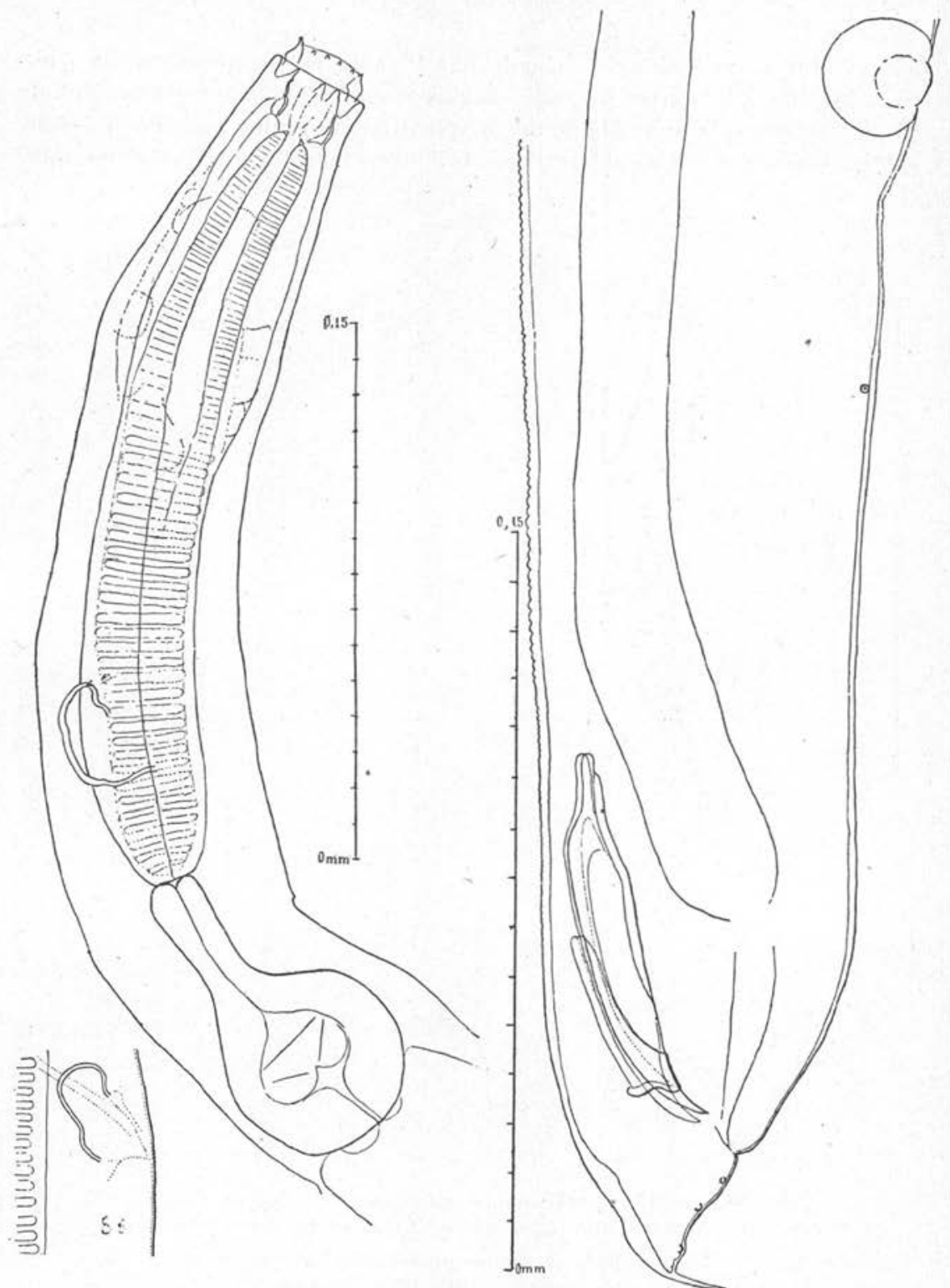

Fig. 85. - Clementeia tubulifera n.sp. Intestin de Leptodesmus jucundus H. W. Brölemann. Descalvado-Pirassununga (Etat de São Paulo, Brésil). Région antérieure d'un $\sigma^{*}$.

FIG. 86. - Même espèce et même provenance que pour la figure 85 . Tubule excréteur d'un autre individu que celui de la fig. 85 .

FIG. 87. - Même espèce et même provenance que pour les figures 85-86. Région postérieure d'un $\delta^{\prime}$ avec spicules longs de $80 \mu$, gubernaculum long de $60 \mu$. 
placas chitinosas nitidas », décrite par P. Artigas. Au niveau de la partie moyenne du dernier tiers du corpus œsophagien, on distingue, chez les 3 spécimens $\delta$ que j'ai à ma disposition, un tube incurvé qui fait partie, vraisemblablement, de l'appareil excréteur terminal. J'ai compté
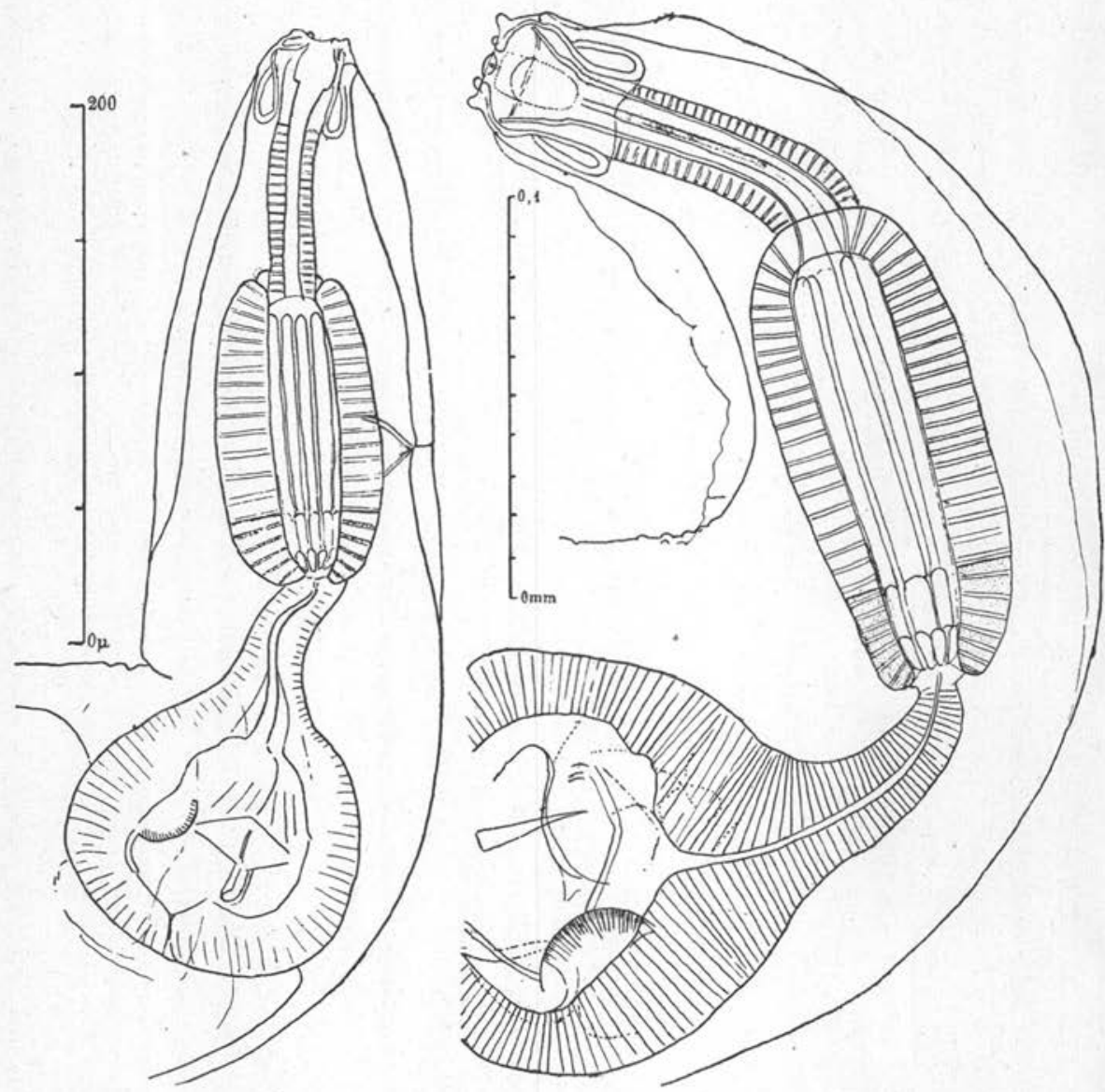

FiG. 88. - Même provenance que pour les figures 85-87.

Région antérieure d'une $\subsetneq$ supposée Clementeia tubulifera mihi.

Fig. 89. - Mème espèce et même provenance que pour la fig. 88 . Partie antérieure du tube digestif d'une $?$.

3 paires de papilles postanales, il existe en outre une paire de papilles à environ $60-66 \mu$ en arrière du milieu de la ventouse préanale, située à environ $220 \mu$ en avant de l'anus.

Un individu, long d'environ $1,035 \mathrm{~mm}$. (dont 0,046 pour la queue), large de 0,10 , a un œsophage long de 0,28 (non compris le goulot du 
bulbe), le goulot du bulbe est long de 0,075 , le diamètre du bulbe mesure 0,07 ; le pore excréteur est à 0,25 de l'extrémité antérieure, et les spicules sont longs de $88 \mu$. Chez un autre individu, les spicules sont longs de $80 \mu$ et le gubernaculum de $37 \mu$.

Provenance : Intestin de Leptodesmus jucundus H. W. Brölemann. Etat de São-Paulo (Brésil).

En même temps que les $\delta$ ci-dessus, se trouvaient quelques $q$ que l'on peut, peut-être, supposer appartenir à la même espèce. Chez Clementeia, les ㅇ ressemblent à celles de Rondonema et de Carnoya à tel point que, quand une $q$ d'un de ces trois genres est trouvée en l'absence de $\delta$, on peut hésiter sur celui des trois auquel elle appartient.

Si les $q$ ci-après décrites n'avaient pas été trouvées en même temps que des ơ de Clementeia, j’aurais été tenté de les attribuer au genre Rondonema.

L'orifice buccal est entouré de 3 lèvres proéminentes, arrondies, portant chacune une papille; la cavité buccale est profonde, un peu en entonnoir. En vue latérale, on voit, au niveau de la moitié postérieure de la cavité buccale, un organe pair, ovale piriforme, à paroi épaisse, qui n'est peut-être qu'un repli disparaissant lors de la protraction de l'extrémité antérieure. Au fond de la cavité buccale aboutit l'œsophage antérieur (précorpus œsophagien = tube pharyngien de G. Gilson), cylindrique, à paroi musculaire, occupant seulement environ le tiers de la largeur du corps à son niveau. Il est suivi par l'œesophage moyen (postcorpus (1) œsophagien = bulbe pharyngien de G. Gilson), manchon musculaire botuliforme, à paroi épaisse, occupant plus de la moitié de la largeur du corps à son niveau. Dans la cavité de ce manchon musculaire se trouvent 6 baguettes œsophagiennes, dont environ le dernier quart est constitué par deux pièces bien délimitées, beaucoup plus courtes, la dernière étant plus courte que l'avant-dernière. (Une disposition très voisine a été décrite par G. Gilson, 1898, p. 348, pl., fig. 16, chez la $q$ de Carnoya vitiensis G. Gilson). A ce postcorpus œsophagien fait suite l'isthme œsophagien, qui est le goulot du bulbe, prolongement antérieur du bulbe, sans démarcation avec la partie

(1) Le terme « metacorpus », proposé pour remplacer \& postcorpus », est un affreux barbarisme que je me refuse à adopter.

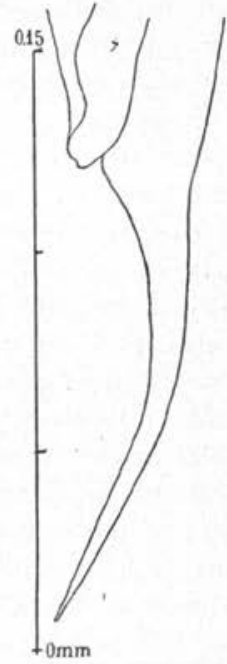

FIG, 90. - Même espèce et même provenave q u e pour les figures $88-89$. Q u e u e d'une $\Varangle$ immature. 
dilatée du bulbe. Le bulbe contient un puissant appareil masticateur.

Chez les $q$ gravides, le corps est fortement renflé dans sa partie moyenne ; j'ai compté environ 18 à 20 œufs utérins, mesurant $142 \times 53 \mu$. La vulve est un peu en arrière de la mi-longueur, non compris la queue ; celle-ci est proportionnellement plus longue que chez Cl. clementei P. Artigas.

Pour une $q$ gravide, longue de $1,8 \mathrm{~mm}$., dont 0,19 pour la queue, large de 0,25 , la vulve est à environ $1 \mathrm{~mm}$. de l'extrémité antérieure et à environ 0,61 de l'anus; chez cette $\&$, le postcorpus œsophagien débute à 0,085 de l'extrémité antérieure du corps, il est long de 0,12, large de 0,055 , l'isthme est long de 0,050 , le bulbe mesure $0,095 \times 0,100$. Chez une + immature, longue de $1,07 \mathrm{~mm}$. (dont environ 0,12 pour la queue), large de 0,13 , le postcorpus œsophagien mesure $0,118 \times 0,063$.

\section{CLEMENTEIA (gen. ??) TRISPICULATA n. sp. (1)}

(Fig. 91-94)

ð. Tête large avec lèvres saillantes. Cavité bucco-pharyngienne courte, large antérieurement, rétrécie postérieurement. Corpus œsophagien botuliforme, s'atténuant légèrement postérieurement, isthme délimité du corpus et moins large que le corpus; cet isthme est le goulot du bulbe, prolongement non délimité de celui-ci. Bulbe avec appareil masticateur, Deux spicules égaux et un gubernaculum. Les spicules, larges antérieurement, s'atténuent en pointe; le gubernaculum, spiculiforme, est un peu plus court que les spicules; il s'élargit légèrement vers la fin de son second tiers, puis s'atténue en pointe. La queue est plutôt courte que longue (elle n'a pas tout à fait un dixième de la longueur totale de l'individu) et s'atténue lentement en pointe; elle porte ventralement une paire de papilles et, dorsalement, un peu plus en avant, une seconde paire de papilles, il existe en outre une ou deux paires de papilles préanales. Il y a une ventouse préanale dont le centre est à environ $152 \mu$ en avant de l'anus.

Un individu, long de $0,910 \mathrm{~mm}$., dont 0,085 pour la queue, large de 0,092, a un corpus œsophagien long d'environ 0,200 , large d'environ 0,051 , un isthme œsophagien long de 0,075 , large de 0,020 , un bulbe de $0,074 \times 0,066$. Les spicules sont longs de $86 \mu$, avec une plus grande largeur de $11,5 \mu$, le gubernaculum est long de $66 \mu$, avec une plus grande largeur de $8,6 \mu$.

Provenance : Intestin de Leptodesmus jucundus H.W. Brölemann. Etat de São-Paulo.

Discussion. - Les deux spécimens à ma disposition ont la partie antérieure en mauvais état; la tête, la cavité bucco-pharyngienne

(1) Allusion à la ressemblance de la forme du gubernaculum avec celle des spicules. 
et le corpus œesophagien sont mal observables, étant en partie masqués par des débris alimentaires. De la cavité buccale d'un des deux spécimens, dépassent trois faisceaux de fins filaments ressemblant un peu à des plumets; on peut supposer que ce sont des corps étrangers, mais je crois plutôt qu'ils appartiennent à la cavité buccale du Nématode.
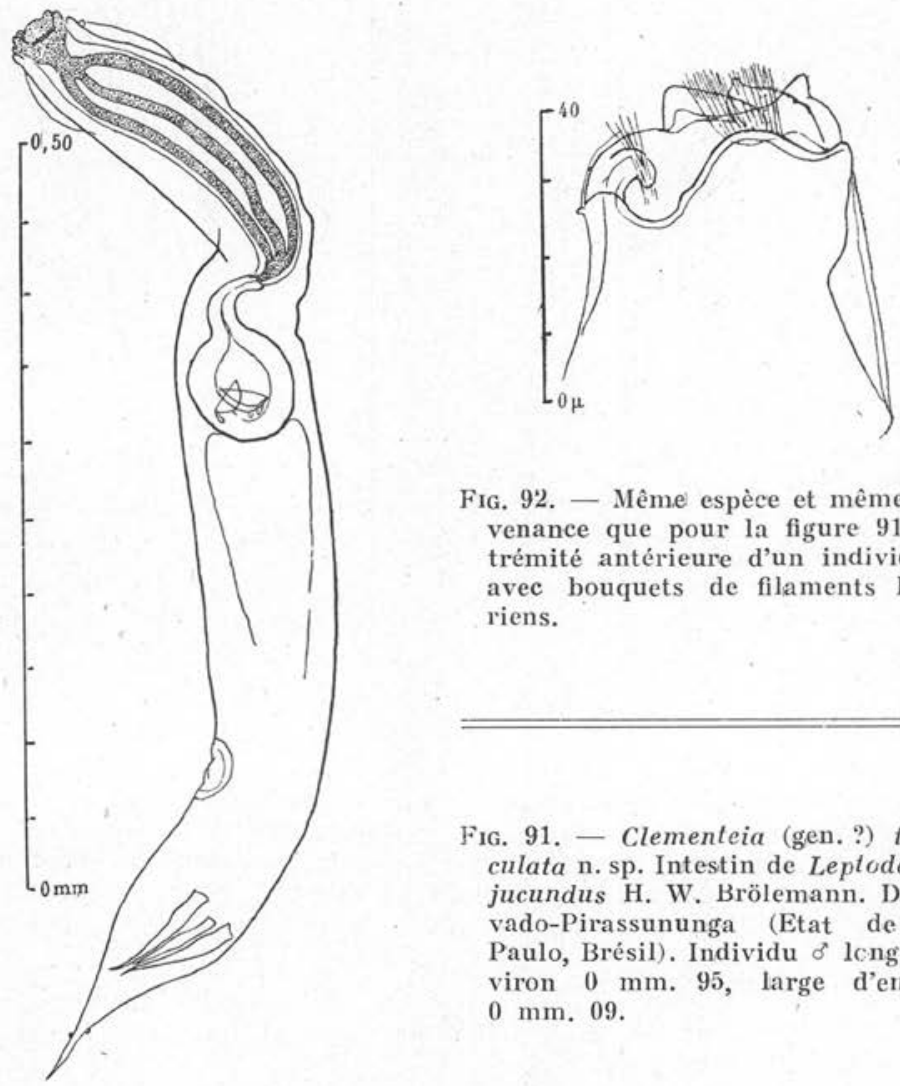

Fig. 92. - Mêmé espèce et même provenance que pour la figure 91. Extrémité antérieure d'un individu $\sigma^{\circ}$, avec bouquets de filaments bactériens.

Fig. 91. - Clementeia (gen. ?) trispiculata $\mathrm{n}$. sp. Intestin de Leptodesmus jucundus H. W. Brölemann. Descalvado-Pirassununga (Etat de São Paulo, Brésil). Individu ơ leng d'environ $0 \mathrm{~mm}$. 95, large d'environ $0 \mathrm{~mm} .09$.

Parmi les genres de Ransomnematinæ à deux spicules égaux + gubernaculum, un seul possède une ventouse préanale : Clementeia, mais l'extrémité antérieure, la forme des spicules, la forme de la région caudale des deux espèces de Clementeia (clementei P. Artigas et tubulifera mihi) diffèrent considérablement de celles de mes deux spécimens, c'est pourquoi leur attribution à ce genre est très douteuse et provisoire ; pour plus de précision, il faudra examiner des spécimens bien conservés; il s'agit vraisemblablement d'un genre nouveau. 


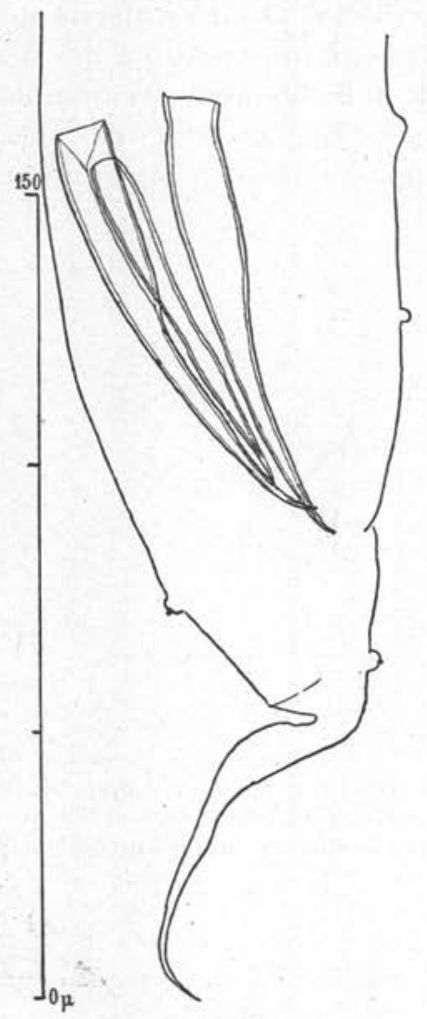

FIG. 93. - Même espèce et même provenance que pour les figures 91 et 92. Région postérieure d'ur. $\delta$ long d'environ $0 \mathrm{~mm}$. 68, large d'environ $0 \mathrm{~mm} .09$.

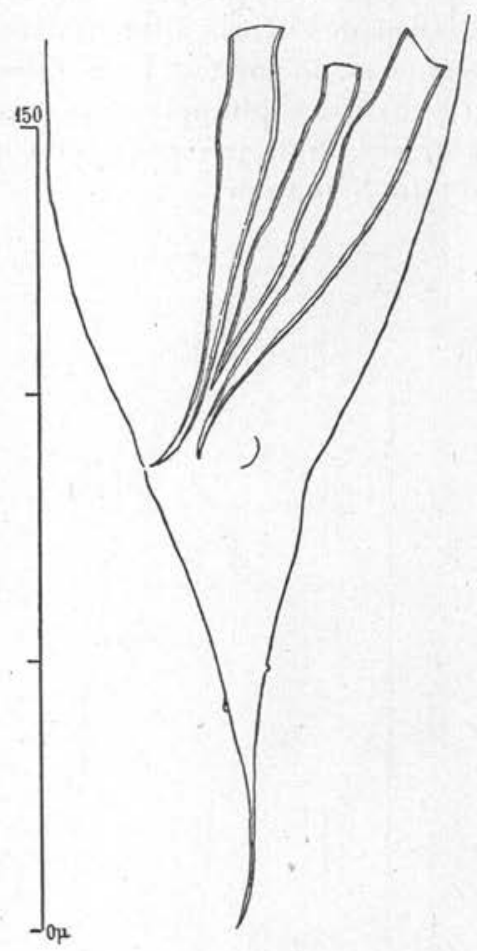

Fig. 94. - Même espèce et même provenance que pour les figures 91-93. Région postérieure de l'individu de la figure 91 .

\section{RÉSUMÉ}

On trouve, chez les Myriapodes, des Nématodes adultes appartenant à des genres qui semblent particuliers aux Myriapodes, des Nématodes adultes de genres représentés aussi chez des Insectes (Blattes, Coléoptères), des Nématodes larvaires pour qui les Myriapodes sont hôte intermédiaire, des Nématodes terricoles ou détriticoles ingérés avec la nourriture, des Nématomorphes (Gordiens). Dans le présent travail, j'ai étudié seulement quelques Nématodes de genres particuliers aux Myriapodes et de genres représentés aussi chez des Insectes. Les spécimens à ma disposition ont été 
récoltés, pour le plus grand nombre, par Mlle Odette Tuzet (1), chez des Myriapodes du Brésil, chez un Myriapode de la Côte d'Ivoire et chez un autre de Sète (Hérault) ; j'ai aussi reçu quelques spécimens récoltés par Jean Théodoridès (2) à Banyuls-sur-Mer (PyrénéesOrientales), et j'en ai moi-même trouvé quelques-uns à Richelieu (Indre-et-Loire).

La faune de Nématodes parasites de Myriapodes, est très peu connue et l'on ne sait pas encore s'il y a des genres ou des espèces de Nématodes dont le parasitisme est limité à une espèce, un genre, une famille de Myriapodes.

\section{BIBLIOGRAPHIE}

1933. Almeida (Jayme Lins de). - Nouveau Nématode parasite de Myriapodes du Brésil : Ichtyocephalus artigasi, n. sp. Compte rendu séances Soc. Biol., Paris, t. CXIV, 1933, p. 1193-1195, fig. 1-2.

1926. Artigas (Paulo). - Nematoides de Invertebrados. I-IV. Boletim Biologico, São Paulo ; fasc. 1 (7-9-1926), p. 1-13, fig. 1-25 ; fasc. 2 (15-10-1926), p. $38-44$, fig. $1-9$; fasc. 3 (15-11-1926), p. 59-71, fig. $1-15$; fasc. 4 (24-121926), p. 97-110, fig. 1-16.

1927. Id. - Nematodeos de Invertebrados. V. Boletim Biologico, São Paulo, fasc. $10,15-12-1927$, p. 209-211, fig. 1-2.

1928. Id. - Nematoides de Invertebrados. VI. Boletim Biologico, São Paulo, fasc. $12,10-7-1928$, p. 71-75, fig. 1-3.

1929. Id. - Systematica dos nematoideos dos arthropodes. These de Doutoramento, Faculdade de Medicina de S. Paulo, 20-12-1929, p. 1-113, pl. IXLV.

1930. Id. - Nematoides dos generos Rhigonema Cobb, 1898, e Dudekemia, n. g. (Nematoda : Rhigonemide, n. n.). Memor. Instit. Oswaldo Cruz, XXIV, fase. 1 , agosto 1930 , p. 19-30, pl. VIII-XIV, fig. 1-32.

1930. Id. - Sobre um novo genero de nematoides, Clementeia, e uma nova especie Clementeia clementei, parasita de julideos (Nematoda: Thelastomida). Memor. Instit. Oswaldo Cruz, XXIV, fasc. 1, agosto 1930, p. 31-34, pl. XV, fig. 1-7.

1942. BAsir (Mohamed Abdul). - Nematodes parasitic in Gryllotalpa. Records Indian Museum, XLIV, part 1, march 1942, p. 92-106, fig. 1a-5e.

1878. BoDE (Johannes). - Polyxenus lagurus De Geer. Ein Beitrag zur Anatomie, Morphologie und Entwicklungsgeschichte der Chilognathen. Zeitschrift für die gesammte Naturwissenschaften. Halle, Bd. XLIX (1877), pl. XI-XIV, Bd. L (1878), p. 233-274. Idem. Dissert. Halis Saxonum, 1878.

(1) Je prie Mlle Odette Tuzet d'agréer mes meilleurs remerciements pour avoir mis à ma disposition ces Nématodes.

(2) Je remercie Jean Théodoridès d'avoir pris la peine de récolter ces spécimens.

Ann. de Parasitologie, T. XXVII, $\mathrm{N}^{\circ} 1-2-3,-1952$. 
1946. Carvalho (José C. M.). - Gordiaceos do Museum de Historia Natural de Montevideo. Comunicaciones zoologicas del Museo de Historia Natural de Montevideo, vol. II, $\mathrm{n}^{\circ} 32$, 1946, p. 1-7, pl. I-IV, fig. 1-36.

1930. Chrtwood (Benjamin, Goodwin). - Description of a new nema parasitic in the intestine of Fontaria. Journ. Parasitol., XVI, n ${ }^{\circ} 3$, march 1930 (Helminthol. Soc. Washington, 122nd meeting, 19-9-1929), p. 163-164, fig. 1-3.

1932. Id. - A synopsis of the nematodes parasitic in Insects of the family Blattidæ. Zeitschrift für Parasitenkunde, V, Heft 1, 18-11-1932, p. 14-50, fig. 1-59 + A-B.

1934. ChItwoon (Benjamin, Goodwin) \& Chirwood (May, Belle). - Nematodes parasitic in Philippine cockroaches. Philippine Journ. of Science, LII, $\mathrm{n}^{\circ} 4$, 8-2-1934, p. 381-393, pl. I-II, fig. 1-19.

1937. Id. \& Id. - An introduction to nematology, Section I, part 1, 53 p., fig. 1$53 \mathrm{M}$. Baltimore, $1937,4^{\circ}$.

1950. Id. \& Id. - An introduction to nematology, Section I, Anatomy, 213 p., fig. 1-145 NN. Baltimore, $1950,4^{\circ}$.

1935. Chitwood (May, Belle). - Two new nematodes of the genus Heth Cobb, 1898 (Atractidæ). Proceed. Helmintholog. Soc. Washington, II, n ${ }^{\circ}$, Jan. 1935, p. 50-51, fig. 8A-8J.

1927. Christie (Jesse, Roy) \& Совв (Nathan, Augustus). - Rhigonema (Isacis Serjabin, 1914 ; ? Isacis Baylis and Daubney, 1926 ; nec Isacis Lespès, 1856), nemas inhabiting the intestine of millipeds. Journ. Washington Acad. Sciences, XVII, n* 1, 3-1-1927, p. 17-19.

1931. Christie (Jesse, Roy). - Some nemic parasitic (Oxyuridæ) of Coleopterous larvæ. Journ. Agricultural Research, Washington, XLII, n ${ }^{\circ}$, 15-4-1931, p. 463-482, fig. 1-14.

1934. Id. - The nematode genera Hystrignathus Leidy, Lepidonema Cobb and Artigasia, n. g. (Thelastomatidx). Proceed. Helminthol. Soc. Washington, I, $\mathrm{n}^{\circ} 2$, July 1934 , p. 44-48, fig. 15A-17.

1938. Id. - A redescription of Thelastoma robustum Leidy with comments on other species of the nematode family Thelastomatidr. Proceed. Helmintholog. Soc. Washington, V, $\mathrm{n}^{\circ} 2$, July 1938, p. 65-67, fig. 22A-22C.

1898. Совв (Nathan, Augustus). - Nematode parasites, their relation to man and domestic animals. Extract from M. S. Report on the parasites of stock. Agricult. Gazette New South Wales, Sydney, IX, $\mathrm{n}^{\circ} 3$, march 1898, p. $296-321$, fig. $1-45 ; n^{\circ} 4$, april 1898 , p. $419-454$, fig. $46-127$.

1929. Id. - Observations on the morphology and physiology of nemas; including notes on a new species. Journ. Washington Acad. Sciences, XIX, $\mathrm{n}^{\circ} 13,19-7-1929$, p. 283-286, fig. 1-7.

1941. Filipjev (Ivan, Nicolas) \& Stekhoven jr (J. H. Schuurmans). - A manual of agricultural helminthology, XV +878 p., fig. $1 a-460 \mathrm{E}$. Leiden, E. J. Brill, 1941.

1879. Gales (Osman). - Recherches sur les Entozoaires des Insectes. Organisation et développement des Oxyurides. Arch. Zoologie expérim. et gén., Paris, sér. 2, vol. VII, 1878, p. 283-290, pl. XVII-XXVI. Id. Thèse Doct. Sc. Nat. Fac. Sciences Paris, série A, $\mathrm{n}^{\circ} 34, \mathrm{n}^{\circ}$ d'ordre 419, 22-31879,108 p., 10 pl.

1898. Guson (Gustave). - Note sur un Nématode nouveau des îles Fidji : Carnoya vitiensis Gilson, nov. gen. La Cellule, Louvain, XIV, fasc. 2, 1898, p. 333-339, pl, fig. 1-23. 
1912. Hagmeier (Arthur). - Beiträge zur Kenntnis der Mermithiden. I. Biologische Notizen und systematische Beschreibung einiger alter und neuer Arten. Zoolog. Jahrbücher System., XXXII, Heft 6, 6-6-1912, p. 521-612, fig. texte A-G, pl. XVII-XXI, fig. 1-55.

1914. Johnston (Thomas, Harvey). - Some new Queensland Entoparasites. Proceed. Royal Soc. Queensland, XXVI, isued 24-11-1914, p. 76-84, pl. IX, fig. 1-13, pl. X, fig. 1-9.

1849. LEIDY (Joseph), - On the existence of Entophyta in healthy animals as a natural condition. Proceed. Acad. Natural Science Philadelphia, vol. IV, $\mathrm{n}^{\circ} 11,9-12-1849$, p. 225-233.

1850. Id. - Description of some nematoid entozoa infesting insects. Proceed. Acad. Natural Science Philadelphia, vol. V, $\mathrm{n}^{\circ} 5$, sept.-oct. 1850 , p. $100-$ 102.

1851. Id. - Corrections and additions to former papers on Helminthology. Proceed. Acad. Natural Science Philadelphia, vol. V, $\mathrm{n}^{\circ} 11$, sept.-oct. 1851, p. 284-290.

1853. Id. - A flora and fauna within living animals. Smithsonian Contributions to Knowledge. Washington, vol. V, art. 2, april 1853, p. 1-67, pl. I-X.

1856. Id. - A Synopsis of the Entozoa and some of their ectocongeners observed by the author. Proceed. Acad. Natural Science Philadelphia, vol. VIII, $\mathrm{n}^{\circ} 1$, jan.-febr. 1856 , p. 42-58.

1878. Linstow (Otto von). - Neue Beobachtungen an Helminthen. Archiv für Naturgesch., XLIV, Bd. I, 1878, p. 218-245, pl. VII-IX, fig. 1-35.

1885. Id. - Beobachtungen an bekannten und neuen Nematoden und Trematoden. Archiv für Naturgesch., LI, Bd. I, 1885, p. 235-255, pl. XIII-XV, fig. 1-31.

1886. Id. - Ueber den Zwischenwirth von Ascaris lumbricoides L. Zoolog. Anzeiger, IX, Nr. 231, 30-8-1886, p. 525-528.

1886. Id. -- Helminthologische Beobachtungen. Archiv für Naturgeschichte, LII, Bd. I, 1886, p. 113-138, pl. VI-IX, fig. 1-34.

1900. Magalhars (Pedro, Severiano de). - Notes d'helminthologie brésilienne. 10. Matériaux pour servir à l'histoire de la flore et de la faune parasitaire de la Periplaneta americana Fabricius. Une nouvelle espèce d'Oxyuris : O. Bulhoesi. Archives Parasitol., Paris, III, $\mathrm{n}^{\circ} 1,15-5-1900$, p. 34-69, fig. 1-25.

1896. MeYer (Adolf). - Neue ceylonische Nematoden aus Säugetieren (Filaria, Strongylus) und aus Julus (Oxyuris). Anatomisch-histologische Untersuchungen. Archiv Naturgesch., LXII, Bd. I, Nr. 1, Juli 1896, p. 54-62, pl. IV-V, fig. 1-25.

1896. Id. - Neue Nematoden unter den Parasiten ceylonesischer Säugetiere und eine Oxyuris, eine neue Schmarotzerspecies in Julus (Ceylon). Anatomisch-histologische Untersuchungen. Inaug. Dissert. philos. Fakultät Univ. Basel, 1898, $35+2$ p., pl. fig. 1-25.

1896. Parona (Corrado). - Di alcuni Nematodi dei diplopodi. Atti Soc. Ligustica Scienze Natur. e Geogr., Genova, VII, n² 2, giugno 1896, p. 108-113, pl. I, fig. 1-19. Id. in Boll. Mus. Zool. e Anat. Compar. Reale Univ. Genova, $\mathrm{n}^{\circ} 44,1896$, p. 1-6, pl. I, fig. 1-19.

1898. Id. - Elminti raccolti dal Dott. Elio Modigliani alle isole Mentawei, Engano e Sumatra. Annali del Museo Civico di Stor. Natur. Genova, vol. XXXIX (2e sér., vol. XIX), 14-7-1898, p. 102-124, pl. I, fig. 1-19. Id. in Boll. Mus. Zool. e Anat. Compar. Reale Univ. Genova, $\mathrm{n}^{\circ} 64,1898$, p. 1-23, pl. I, fig. 1-19. 
1891. Rath (Otto vom). - Zur Biologie der Diplopoden. Berichte der naturforsch. Gesellsch. zu Freiburg i. Brisgau, Bd. V, Heft 2, 1891, p. 161-199.

1916. Rall.liet (Alcide) et Henry (Albert). - Sur les Oxyuridés. Compte rendu séances Soc. Biologie, Paris, t. LXXIX, séance du 5-2-1916, p. 113-115.

1916. Id. - Nouvelles remarques sur les Oxyuridés. Compte rendu séances Soc. Biologie, Paris, t. LXXIX, séance du 1-4-1916, p. 247-250.

1946. Sanchez (Amparo, Serrano). - Nematodes parasitos intestinales de artropódos en España. Tesis Doctoral (26-11-1946), Granada, 66 p., pl. I-IX.

1947. Id. - Nematodes parasitos intestinales de los artropódos en España. Revista Iberica de Parasitologia, Granada, VIII, n² 2, 1947, p. 279-332, pl. I-IX.

1947. Schubart (Otto). - Os Diplopoda da viagen do naturalista Antenon Leitão de Carvalho aos rios Araquaia e Amazonas em 1939 e 1940. Boletim du Museu Nacional, Rio de Janeiro. Nova série. Zoologica, $\mathrm{n}^{\circ} 82,30-8-1947$, p. 1-74, pl. I-XXVII, fig. 1-75, photogr. 1-6.

1926. Schwenck (Jules). - Fauna parasitologica dos blattiden do Brasil. Sciencia Medica, Rio de Janeiro, IV, n 9, 30-9-1926, p. 491-504, fig. 1-20.

1927. Id. - Sobre um Nematoide parasita de Oniscidx. Boletim biologico, São Paulo, fasc. 7, 20-6-1927, p. 78-85, fig. 1-11.

1916. SkRJABIN (Konstantin, Ivanovitch). - Parasitic Trematodes and Nematodes collected by the Expeditions of Prof. V. Dogiel and I. Sokolow in British East Africa. Expeditions of Prof. V. Dogiel and I. Sokolow in British East Africa. Zoological Results, $\mathrm{n}^{\circ} 4$, Petrograd, 1916, p. 1-157, fig. texte 1-2, pl. I-X, fig. 1-82.

1927. Id. - Sur deux nouveaux Nématodes parasites des Myriapodes. Annuaire Musée Zoolog. Acad. Sciences de l'U.R.S.S., XXVII (1926), n ${ }^{\circ} 4,1927$, p. 304-309, fig. 1-6.

1930. Thомas (Lyell J.). - Rhigonema nigella, spec. nov., a nematode and its plant commensal. Enterobrus sp. ? from the milliped. Journ. Parasitol., XVII, $\mathrm{n}^{\circ}$ 1, 4-10-1930, p. 30-34, pl. III, fig. 1-12 ; pl. IV, fig. 13-18.

1919. Travassos (Lauro). - Esboģo de uma chave geral dos nematodes parasitos. Revista de Veterinaria e Zootechnia, Rio de Janeiro, anno $\mathrm{X}, \mathrm{n}^{\circ} 2$, 1920 , p. $59-70+1$ table +1 tableau.

1929. Id. - Contribução preliminar a systematica dos nematodeos dos arthropodos. Instituto Osualdo Cruz, Supplemento das Memorias, $\mathrm{n}^{\circ} 5$, jan.fev. 1929, p. 19-25, pl. fig. 1-12.

1859. UneкEм (Jules d'). - Notice sur quelques parasites de Julius terrestris. Bull. Acad. Royale des Sciences, Belgique, sér. 2, t. VII, séance du 8-81859 , p. 552-567, pl. I, fig. 1-12; pl. II, fig. 1-9.

1928. WALton (Arthur C.). - A revision of the Nematodes of the Leidy collection. Proceed. Acad. Nat. Sciences Philadelphia, vol, LXXIX (1927), 14-1-1928, p. 49-163, pl. IV-X, fig. 1-92.

1910. Wernitzsch (W.). - Beiträge zur Kenntnis von Craspedosoma simile und des Tracheensystems der Diplopoden. Jenaüsche Zeitschnift für Naturwissenschaft, Bd. XLVI (N.F. XXXIX), Heft 1, 1910, p. 225-284, fig. 1-16, pl. XI, fig. 1-16, ; pl. XII, fig. 17-30. 